BUTP-2000-01

MPI-PhT/2000-01

\title{
The Intrinsic Coupling in Integrable Quantum Field Theories
}

\author{
J. Balog ${ }^{1}$, M. Niedermaier ${ }^{2}$, F. Niedermayer ${ }^{3}$ \\ A. Patrascioiu ${ }^{4}$, E. Seiler ${ }^{5}$, P. Weisz ${ }^{5}$ \\ ${ }^{1}$ Research Institute for Particle and Nuclear Physics \\ 1525 Budapest 114, Hungary \\ ${ }^{2}$ Department of Physics, University of Pittsburgh \\ Pittsburgh, PA 15260, U.S.A. \\ ${ }^{3}$ Institute for Theoretical Physics, University of Bern \\ CH-3012 Bern, Switzerland \\ ${ }^{4}$ Physics Department, University of Arizona \\ Tucson, AZ 85721, U.S.A. \\ ${ }^{5}$ Max-Planck-Institut für Physik \\ 80805 Munich, Germany
}

\begin{abstract}
The intrinsic 4-point coupling, defined in terms of a truncated 4-point function at zero momentum, provides a well-established measure for the interaction strength of a QFT. We show that this coupling can be computed nonperturbatively and to high accuracy from the form factors of an (integrable) QFT. The technique is illustrated and tested with the Ising model, the XYmodel and the $\mathrm{O}(3)$ nonlinear sigma-model. The results are compared to those from high precision lattice simulations.
\end{abstract}




\section{Introduction}

The intrinsic coupling $g_{\mathrm{R}}$, also sometimes called 'physical' or 'renormalized' coupling, is a quantity of great interest in a Quantum Field Theory (QFT), especially for scalar fields. In some cases, such as the $\Phi^{4}$ theories, its vanishing implies actually that the theory is trivial in the sense that the higher correlation functions of the scalar field can be written as sums of products of two point functions, as in a free theory [四]. On the other hand, a non-vanishing $g_{\mathrm{R}}$ is not sufficient to assure the non-triviality of a theory; it only assures that a certain four point vertex function does not vanish identically, but does not exclude that it vanishes on shell.

Aside from that, $g_{\mathrm{R}}$ is certainly a renormalization group invariant and a characteristic physical quantity of a field theory. In particular it can be used to check the equivalence or nonequivalence of different definitions of theories; this will be our main theme in this paper. $g_{\mathrm{R}}$ is proportional to the connected - sometimes called truncated - four point function at zero momentum, divided by the square of the zero momentum two point function and appropriate powers of the mass gap to make it dimensionless; details will be given in the body of the paper.

We are dealing in this article with two main approaches to the construction of a QFT. The first one starts from a suitably regularized functional integral and then removes the regularization in a controlled way. This is a rather general procedure usable for a wide variety of models; it has been successfully employed to construct QFTs in 2 and 3 dimensions obeying all the required axioms (see for instance [2]). Here we will make use of a Euclidean space-time lattice as a regulator. Removal of the regularization, i.e. taking the continuum limit in a lattice theory requires the existence of a second order phase transition point at which the characteristic length (correlation length) of the model diverges. This approach raises the problem of 'universality', i.e. the question whether different regularizations yield the same QFT after the regulator has been removed.

The other approach studied here is applicable to a large class of so-called integrable models. It is not based on a Lagrangian, rather the dynamics is specified in terms of a postulated exact 'bootstrap' S-matrix, supposed to enjoy a factorization property that allows to express all S-matrix elements in terms of the two-particle S-matrix [3]. In physical terms this property is linked to the existence of an infinite number of conservation laws and the absence of particle production. The postulated S-matrices are then used to set up a system of recursive functional equations for the form factors; solving this system one can in principle compute exactly all the form factors, in other words continue the 
S-matrix off the mass shell 4 , 5, 6]. Once the form factors are known, one can express the correlation functions of the basic fields as well as other (composite) operators by inserting complete sets of scattering states between them. This gives the correlation functions as hopefully rapidly converging - infinite series of convolution products of form factors. In particular in this way one can express the intrinsic coupling in terms of the form factors.

In both approaches, in principle one has to verify in the end that the axioms of a QFT hold. In the lattice approach with a reflection positive action, such as the standard nearest neighbor action, essentially the only nontrivial question besides the existence of a critical point concerns the restoration of Euclidean (Poincaré) invariance in the continuum limit. In the form factor approach it is less obvious whether the axioms hold, in particular for the form factor expansion of multi-point correlation functions. There exists, however, a formal proof (disregarding convergence aspects) of locality [6] and it is hoped, of course, that the other field theoretic axioms will hold as well, because the construction is to a large extent inspired by them. It is also not clear from first principles - though in practice there are very natural guesses -, which field in one construction should be identified with which form factor sequence in the other. In any case, even assuming that all the axioms hold in both constructions and that one has correctly identified the fields, it is a nontrivial question whether the two approaches define the same theory, and in particular whether they give the same value for $g_{\mathrm{R}}$.

In the lattice approach the intrinsic coupling of the two-dimensional $\mathrm{O}(n)$ models we are discussing here has been widely studied, both by Monte Carlo simulations [7, 8, 9] and by various expansions in a small parameter [10, 11, 12, 13]. For a more precise comparison with the form factor approach, we also carried out our own high precision Monte Carlo simulations which are reported in this paper.

In the form factor approach the series for $g_{\mathrm{R}}$, being a low energy quantity, is expected to converge very rapidly. Our results give every indication that these hopes are fully justified, though the actual computations turn out to be surprisingly intricate. In the present study we want to develop this computational framework, outline the computations and compare their results, where possible, to those obtained numerically from the lattice approach by the different methods mentioned above.

Remarkably in all the examples considered the first non-trivial term in the series, which contains only one and two particle intermediate states, appears to give about $98 \%$ of the full answer (!). Moreover for this dominant contribution a general model-independent expression in terms of the 1-and 3-particle form factors and the derivative of the S-matrix 
can be obtained.

In this paper we discuss three models which can be viewed as the $\mathrm{O}(n)$ nonlinear sigmamodels for $n=1,2,3$. Though formally members of the $\mathrm{O}(n)$ series of nonlinear sigmamodels, the physics of these systems, their form factor description, and not the least our motivation to study them is very different: The $n=1$ case is just the massive continuum limit of the Ising model. Here the spin form factors are very simple and we were able to push the computation of the series up to all terms with a total particle number (summed over the three intermediate states) of less or equal 8 . The extremely rapid decay of the terms is manifest and we use the observed pattern as a guideline for the other systems. The final result amounts to a determination of $g_{\mathrm{R}}$ with an estimated precision of better than $0.001 \%$.

The $n=2$ case is better known as the XY-model. Here we rely on a bootstrap description of the model, to which we hope to return in more detail elsewhere [14]. Not all the form factors are known explicitly, but the specific version of the 3-particle spin form factor needed for the dominant contribution can be found by elementary techniques. We compare this leading order result with that obtained by lattice techniques and find reasonable agreement, which can be taken as support for the proposed bootstrap description.

Finally the $n=3$ model is the first with a nonabelian symmetry group. The evaluation of $g_{\mathrm{R}}$ here is in part motivated by the controversy about the absence or presence of a Kosterlitz-Thouless type phase transition; see [9] for a more thorough discussion.

Let us remark that the form factor bootstrap has also been applied to the computation of $g_{\mathrm{R}}$ in the sinh-Gordon model; in this model the intrinsic coupling is especially interesting because of its relevance to the issue of "triviality" versus "weak-strong-duality". For details see the accompanying paper [15].

The article is organized as follows. In the next section we describe the form factor construction of Green's functions in terms of form factors generally and derive the formula for the dominant contribution to the coupling. Further we prepare the ground for the computation of the sub-leading terms in the specific models. We then give a few generalities about the Monte Carlo simulations, and move on to discuss the three models as outlined above one by one in more detail, comparing the results of the form factor construction to those obtained by the the lattice definition of the models; for the latter the values of $g_{\mathrm{R}}$ are estimated by high temperature expansions as well as Monte Carlo simulations. 


\section{Construction of Green functions in terms of form factors}

In this section we will consider a general massive QFT described in terms of its generalized form factor sequences by which we mean matrix elements of local operators between physical states. We will restrict our attention to the case of $d=2$ dimensions (although the extension to arbitrary $d$ is often straightforward). The application of the representation to the integrable models where the form factors are explicitly known will be the subject of the next chapter.

\subsection{Generalities}

Our first goal is to construct the Euclidean correlation functions (Schwinger functions) from the generalized form factors. The Schwinger functions are convenient because they have simpler properties than the Wightman functions and also because it facilitates the comparison with lattice results later. For points $x_{k} \in \mathbb{R}^{2}, k=1, \ldots, L$, we denote by $\left(x_{k 1}, x_{k 2}\right)$ their components and by $\iota x_{k}=\left(-i x_{k 2}, x_{k 1}\right)$ a Wick rotated version. For definiteness we will consider here correlation functions of $n$ scalar fields $\Phi^{a}(x), a=1, \ldots, n$ (the generalization to other types of fields is straightforward). Then

$$
\begin{aligned}
& S^{a_{1} \ldots a_{L}}\left(x_{1}, \ldots, x_{L}\right)=\left\langle\Phi^{a_{1}}\left(x_{1}\right) \ldots \Phi^{a_{L}}\left(x_{L}\right)\right\rangle \\
& S^{a_{1} \ldots a_{L}}\left(x_{1}, \ldots, x_{L}\right)=W^{a_{1} \ldots a_{L}}\left(\iota x_{1}, \ldots, \iota x_{L}\right) \text { for } x_{12}>\ldots>x_{L 2} .
\end{aligned}
$$

The first equation is the usual operator interpretation of the Schwinger functions. The second equation (2.1) then indicates the relation of the Schwinger function to the corresponding Wightman function for points $\left(z_{1}, \ldots, z_{L}\right)=\left(\iota x_{1}, \ldots, \iota x_{L}\right)$ in the "primitive

tube" of analyticity. . Outside the primitive tube the Schwinger functions can in principle likewise be obtained from the Wightman functions by analytic continuation and are then found to be completely symmetric in all variables. In a form factor expansion however the primitive domain is preferred in that only there the convergence of the momentum space integrals is manifest through exponential damping factors (c.f. below). We thus mimic the effect of the analytic continuation by performing the symmetrization by hand

$$
\begin{aligned}
& S^{a_{1} \ldots a_{L}}\left(x_{1}, \ldots, x_{L}\right)=\sum_{s \in \mathcal{S}_{L}} S_{\Theta}^{a_{s 1} \ldots a_{s L}}\left(x_{s 1}, \ldots, x_{s L}\right) \\
& S_{\Theta}^{a_{1} \ldots a_{L}}\left(x_{1}, \ldots, x_{L}\right):=\Theta\left(x_{1}, \ldots, x_{L}\right) W^{a_{1} \ldots a_{L}}\left(\iota x_{1}, \ldots, \iota x_{L}\right),
\end{aligned}
$$

*We use the signature $(+,-)$ for (complexified) Minkowski space in which case $\left(z_{1}, \ldots, z_{L}\right)$ is in the primitive tube iff $-\operatorname{Im}\left(z_{k}-z_{k+1}\right) \in V^{+}, k=1, \ldots, L-1$, where $V^{+}$is the forward light cone. 
where $\Theta\left(x_{1}, \ldots, x_{L}\right)$ is a generalized step function that vanishes unless $x_{12} \geq \ldots \geq x_{L 2}$ holds and the sum is over all elements of the permutation group $\mathcal{S}_{L}$. The functions $S_{\Theta}^{a_{1} \ldots a_{L}}\left(x_{1}, \ldots, x_{L}\right)$ are expected to have a convergent expansion in terms of form factors in the interior of their support region (as well as for certain points on the boundary). The cases of interest here are $L=2$ and $L=4$. Formally inserting a resolution of the identity in terms of asymptotic multi-particle states $\mathbb{1}=\sum_{\underline{m}}|\underline{m}\rangle\langle\underline{m}|$ one obtains

$$
S_{\Theta}^{a_{1} a_{2}}\left(x_{1}, x_{2}\right)=\Theta\left(x_{1}, x_{2}\right) \sum_{\underline{m}} e^{-\left(x_{1}-x_{2}\right)_{2} E_{\underline{m}}} e^{i\left(x_{1}-x_{2}\right)_{1} P_{\underline{m}}}\left\langle 0\left|\Phi^{a_{1}}(0)\right| \underline{m}\right\rangle\left\langle\underline{m}\left|\Phi^{a_{2}}(0)\right| 0\right\rangle,
$$

and

$$
\begin{aligned}
S_{\Theta}^{a_{1} a_{2} a_{3} a_{4}}\left(x_{1}, x_{2}, x_{3}, x_{4}\right)= & \Theta\left(x_{1}, x_{2}, x_{3}, x_{4}\right) \sum_{\underline{k}, \underline{l}, \underline{m}} e^{-\left(x_{1}-x_{2}\right)_{2} E_{\underline{k}}} e^{i\left(x_{1}-x_{2}\right)_{1} P_{\underline{k}}} \\
& e^{-\left(x_{2}-x_{3}\right)_{2} E_{\underline{l}}} e^{i\left(x_{2}-x_{3}\right)_{1} P_{\underline{l}}} e^{-\left(x_{3}-x_{4}\right)_{2} E_{\underline{m}}} e^{i\left(x_{3}-x_{4}\right)_{1} P_{\underline{m}}} \\
& \left\langle 0\left|\Phi^{a_{1}}(0)\right| \underline{k}\right\rangle\left\langle\underline{k}\left|\Phi^{a_{2}}(0)\right| \underline{l}\right\rangle\left\langle\underline{l}\left|\Phi^{a_{3}}(0)\right| \underline{m}\right\rangle\left\langle\underline{m}\left|\Phi^{a_{4}}(0)\right| 0\right\rangle .
\end{aligned}
$$

The states $|\underline{m}\rangle$ are assumed to be improper eigenstates of the momentum operator $P_{\mu}$, and $E_{\underline{m}}, P_{\underline{m}}$ denote the eigenvalues of $P_{0}, P_{1}$ on $|\underline{m}\rangle$, respectively. To write down an explicit parameterization of the complete set of states $|\underline{m}\rangle$ requires of course the full knowledge of the spectrum of stable particles. This is a basic input assumption for the integrable models dealt with in the next section. Here for simplicity of notation we will consider the case where there is only one multiplet of stable particle states of mass $M$. An explicit parameterization will then be given in subsection 2.3.

We introduce their (dimensionless) Fourier transforms $V$ by

$$
\begin{aligned}
& (2 \pi)^{2} \delta^{(2)}\left(k_{1}+\ldots+k_{L}\right) M^{-2(L-1)} V^{a_{1} \ldots a_{L}}\left(k_{1}, \ldots, k_{L}\right) \\
& \quad=\int \mathrm{d}^{2} x_{1} \ldots \mathrm{d}^{2} x_{L} S_{\Theta}^{a_{1} \ldots a_{L}}\left(x_{1}, \ldots, x_{L}\right) e^{i\left(k_{1} x_{1}+\ldots+k_{L} x_{L}\right)},
\end{aligned}
$$

taking into account the translation invariance of $S_{\Theta}$. The Fourier transform of the full Schwinger function is then obtained by symmetrization

$$
\tilde{S}^{a_{1} \ldots a_{L}}\left(k_{1}, \ldots, k_{L}\right)=(2 \pi)^{2} \delta^{(2)}\left(k_{1}+\ldots+k_{L}\right) M^{-2(L-1)} \sum_{s \in \mathcal{S}_{L}} V^{a_{s 1} \ldots a_{s L}}\left(k_{s 1}, \ldots, k_{s L}\right)
$$

whereon rotational invariance gets restored. The desired representation of the two and four point functions in terms of form factors is given by

$$
V^{a_{1} a_{2}}\left(k_{1}, k_{2}\right)=\sum_{\underline{m}} V_{\underline{m}}^{a_{1} a_{2}}\left(k_{1}, k_{2}\right),
$$




$$
V^{a_{1} a_{2} a_{3} a_{4}}\left(k_{1}, k_{2}, k_{3}, k_{4}\right)=\sum_{\underline{k}, \underline{l,}, \underline{m}} V_{\underline{k l m}}^{a_{1} a_{2} a_{3} a_{4}}\left(k_{1}, k_{2}, k_{3}, k_{4}\right)
$$

where

$$
\begin{aligned}
V_{\underline{m}}^{a_{1} a_{2}}\left(k_{1}, k_{2}\right)= & 2 \pi M^{2} \frac{\delta\left(P_{\underline{m}}+k_{11}\right)}{E_{\underline{m}}-i k_{12}}\left\langle 0\left|\Phi^{a_{1}}(0)\right| \underline{m}\right\rangle\left\langle\underline{m}\left|\Phi^{a_{2}}(0)\right| 0\right\rangle, \\
V_{\underline{k l m}}^{a_{1} a_{2} a_{3} a_{4}}\left(k_{1}, k_{2}, k_{3}, k_{4}\right)= & \left(2 \pi M^{2}\right)^{3} \frac{\delta\left(P_{\underline{k}}+k_{11}\right)}{E_{\underline{k}}-i k_{12}} \frac{\delta\left(P_{\underline{l}}+k_{11}+k_{21}\right)}{E_{\underline{l}}-i k_{12}-i k_{22}} \frac{\delta\left(P_{\underline{m}}-k_{41}\right)}{E_{\underline{m}}+i k_{42}} \\
& \left\langle 0\left|\Phi^{a_{1}}(0)\right| \underline{k}\right\rangle\left\langle\underline{k}\left|\Phi^{a_{2}}(0)\right| \underline{l}\right\rangle\left\langle\underline{l}\left|\Phi^{a_{3}}(0)\right| \underline{m}\right\rangle\left\langle\underline{m}\left|\Phi^{a_{4}}(0)\right| 0\right\rangle,
\end{aligned}
$$

with the understanding that the sum of the momenta $k_{j}$ vanishes. Further we denote by $V_{m}^{a_{1} a_{2}}\left(k_{1}, k_{2}\right)$ and $V_{k l m}^{a_{1} a_{2} a_{3} a_{4}}\left(k_{1}, k_{2}, k_{3}, k_{4}\right)$ the quantities (2.8) with the integrations over the rapidities performed, the measure being inherited from Eq. (2.25) below.

The key assumption of the form factor approach in this context is that the matrix elements in (2.8) can be computed exactly via solutions of a recursive system of functional equations, the so-called form factor equations or Smirnov axioms. Symbolically

$$
\left\langle\underline{l}\left|\Phi^{a}(0)\right| \underline{m}\right\rangle \quad \longleftrightarrow \quad \mathcal{F}_{b_{1} \ldots b_{l} a_{1} \ldots a_{m}}^{a}\left(\omega_{1}, \ldots, \omega_{l} \mid \theta_{1}, \ldots, \theta_{m}\right)=: \mathcal{F}_{B A}^{a}(\omega \mid \theta)
$$

The rhs, for which we shall often use the indicated shorthand notation, is called a generalized form factor, the special case with either $l=0$ or $m=0$ are the form factors proper. The form factors are meromorphic functions in the rapidities, while the generalized form factors are distributions. The form factors can be computed, at least in principle, as solutions of the before mentioned system of functional equations. The generalized form factors can then be obtained from them by means of an explicit, though cumbersome, combinatorial formula. We shall later just state the special cases of this formula required. A discussion of the general formula can e.g. be found in the appendix of [16].

Implicit in the products of matrix elements in (2.8) of course are appropriate index contractions. For definiteness let us note them explicitly

$$
\begin{aligned}
\left\langle 0\left|\Phi^{a}(0)\right| \underline{m}\right\rangle\left\langle\underline{m}\left|\Phi^{b}(0)\right| 0\right\rangle & \longleftrightarrow I_{m}^{a b}(\theta), \\
\left\langle 0\left|\Phi^{a}(0)\right| \underline{k}\right\rangle\left\langle\underline{k}\left|\Phi^{b}(0)\right| \underline{l}\right\rangle\left\langle\underline{l}\left|\Phi^{c}(0)\right| \underline{m}\right\rangle\left\langle\underline{m}\left|\Phi^{d}(0)\right| 0\right\rangle & \longleftrightarrow I_{k l m}^{a b c d}(\omega|\xi| \theta),
\end{aligned}
$$

where

$$
\begin{aligned}
& I_{m}^{a b}(\theta)=\sum_{A} \mathcal{F}_{A}^{a}(\theta) \mathcal{F}_{A^{T}}^{b}\left(\theta^{T}\right) \\
& I_{k l m}^{a b c d}(\omega|\xi| \theta)=\sum_{A, B, C} \mathcal{F}_{A}^{a}(\omega) \mathcal{F}_{A^{T} B}^{b}\left(\omega^{T} \mid \xi\right) \mathcal{F}_{B^{T} C}^{c}\left(\xi^{T} \mid \theta\right) \mathcal{F}_{C^{T}}^{d}\left(\theta^{T}\right)
\end{aligned}
$$


Here $A^{T}=\left(a_{k}, \ldots, a_{1}\right), \omega^{T}=\left(\omega_{k}, \ldots, \omega_{1}\right)$, etc. The construction is such that $I_{m}^{a b}(\theta)$ is a completely symmetric function in $\theta=\left(\theta_{1}, \ldots, \theta_{m}\right)$. Similarly $I_{k l m}^{a b c d}(\omega|\xi| \theta)$ is symmetric in each of the sets of variables $\omega=\left(\omega_{1}, \ldots, \omega_{k}\right), \xi=\left(\xi_{1}, \ldots, \xi_{l}\right)$ and $\theta=\left(\theta_{1}, \ldots, \theta_{m}\right)$, individually.

\subsection{The intrinsic coupling}

As surveyed in the introduction the intrinsic coupling is defined in terms of the zero momentum limit of a connected 4-point function. We may assume that the Schwinger functions of the scalar fields with an odd number of arguments vanish; then the connected $L=2,4$ Schwinger functions of interest here are

$$
\begin{aligned}
\tilde{S}_{c}^{a_{1} a_{2}}\left(k_{1}, k_{2}\right) & =\tilde{S}^{a_{1} a_{2}}\left(k_{1}, k_{2}\right) \\
\tilde{S}_{c}^{a_{1} a_{2} a_{3} a_{4}}\left(k_{1}, k_{2}, k_{3}, k_{4}\right) & =\tilde{S}^{a_{1} a_{2} a_{3} a_{4}}\left(k_{1}, k_{2}, k_{3}, k_{4}\right)-\tilde{S}^{a_{1} a_{2}}\left(k_{1}, k_{2}\right) \tilde{S}^{a_{3} a_{4}}\left(k_{3}, k_{4}\right) \\
& -\tilde{S}^{a_{1} a_{3}}\left(k_{1}, k_{3}\right) \tilde{S}^{a_{2} a_{4}}\left(k_{2}, k_{4}\right)-\tilde{S}^{a_{1} a_{4}}\left(k_{1}, k_{4}\right) \tilde{S}^{a_{2} a_{3}}\left(k_{2}, k_{3}\right)
\end{aligned}
$$

Making explicit the overall delta-functions arising from translational invariance we introduce the Green functions by

$$
\tilde{S}_{c}^{a_{1} \ldots a_{L}}\left(k_{1}, \ldots, k_{L}\right)=(2 \pi)^{2} \delta^{(2)}\left(k_{1}+\ldots+k_{L}\right) G^{a_{1} \ldots a_{L}}\left(k_{1}, \ldots, k_{L}\right)
$$

where the constraint $k_{1}+\ldots+k_{L}=0$ in the arguments of $G^{a_{1} \ldots a_{L}}$ will always be understood.

In the following we will now assume that the theory is $\mathrm{O}(n)$ invariant and thus for the 2-point function we can write

$$
G^{a_{1} a_{2}}(k,-k)=\delta^{a_{1} a_{2}} G(k)
$$

The intrinsic coupling is then defined by

$$
g_{\mathrm{R}}=-\mathcal{N} \frac{M^{2}}{G(0)^{2}} \frac{1}{n^{2}} \sum_{a, b} G^{a a b b}(0,0,0,0)
$$

where we leave the choice of positive constant $\mathcal{N}$ for later. 
Performing the symmetrization (2.2) and the Fourier transform one recovers the familiar expression for $G(k)$ in terms of the spectral density

$$
G(k)=\int_{0}^{\infty} \mathrm{d} \mu \rho(\mu) \frac{1}{\mu^{2}+k^{2}}
$$

where

$$
\rho(\mu)=\sum_{\underline{m}} \delta\left(\mu-\sqrt{E_{\underline{m}}^{2}-P_{\underline{m}}^{2}}\right) 4 \pi E_{\underline{m}} \delta\left(P_{\underline{m}}\right) \frac{1}{n} \sum_{a}\left\langle 0\left|\Phi^{a}(0)\right| \underline{m}\right\rangle\left\langle\underline{m}\left|\Phi^{a}(0)\right| 0\right\rangle .
$$

In order to compute $\tilde{S}^{a_{1} a_{2} a_{3} a_{4}}\left(k_{1}, k_{2}, k_{3}, k_{4}\right)$ the symmetrized sum (2.6), (2.7) has to be performed. For reasons that will become clear immediately we first single out the partial sum with $\underline{l}=0$. Taking into account the $\mathcal{S}_{4}$ permutations one finds

$$
\begin{aligned}
& (2 \pi)^{2} \delta^{(2)}\left(k_{1}+k_{2}+k_{3}+k_{4}\right) M^{-6} \sum_{\underline{k}, \underline{m}} \sum_{s \in \mathcal{S}_{4}} V_{\underline{k} 0 \underline{m}}^{a_{1} a_{2} a_{3} a_{4}}\left(k_{s 1}, k_{s 2}, k_{s 3}, k_{s 4}\right) \\
& =\tilde{S}^{a_{1} a_{2}}\left(k_{1}, k_{2}\right) \tilde{S}^{a_{3} a_{4}}\left(k_{3}, k_{4}\right)+\tilde{S}^{a_{1} a_{3}}\left(k_{1}, k_{3}\right) \tilde{S}^{a_{2} a_{4}}\left(k_{2}, k_{4}\right)+\tilde{S}^{a_{1} a_{4}}\left(k_{1}, k_{4}\right) \tilde{S}^{a_{2} a_{3}}\left(k_{2}, k_{3}\right) \\
& +(2 \pi)^{2} \delta^{(2)}\left(k_{1}+k_{2}+k_{3}+k_{4}\right) M^{-6} \sum_{s \in \mathcal{S}_{4}} \Omega^{a_{s 1} a_{s 2} a_{s 3} a_{s 4}}\left(k_{s 1}, k_{s 2}, k_{s 3}, k_{s 4}\right) .
\end{aligned}
$$

Here

$$
\Omega^{a_{1} a_{2} a_{3} a_{4}}\left(k_{1}, k_{2}, k_{3}, k_{4}\right)=-\frac{\pi}{2} \delta\left(k_{11}+k_{21}\right) M^{6} \delta^{a_{1} a_{2}} \delta^{a_{3} a_{4}} H\left(k_{1}, k_{2}\right) G\left(k_{4}\right),
$$

with

$$
H\left(k_{1}, k_{2}\right)=\int_{0}^{\infty} \mathrm{d} \mu \rho(\mu) \frac{\mu^{2}+k_{11}^{2}+k_{12} k_{22}}{\left(\mu^{2}+k_{1}^{2}\right)\left(\mu^{2}+k_{2}^{2}\right)} \frac{1}{\sqrt{\mu^{2}+k_{11}^{2}}} .
$$

In obtaining (2.18) we defined the second denominator in (2.8) for $\underline{l}=0$ with the $i \epsilon$ prescription as: $-i\left(k_{12}+k_{22}+i \epsilon\right)$. Here and later the distributional identity

$$
\frac{1}{x+i \epsilon}=\mathcal{P} \frac{1}{x}-i \pi \delta(x)
$$

will be heavily used, where $\mathcal{P}$ is the Principal Value prescription.

One observes that the first three terms in (2.18) are precisely the ones removed by the definition of the connected 4-point function. Remarkably there is a remainder, the $\Omega$ term, which is present even in the free theory. Typically the spectral densities are decreasing or 
bounded by a constant as $\mu \rightarrow \infty$. The functions $G\left(k_{4}\right)$ and $H\left(k_{1}, k_{2}\right)$ are then regular at $k_{i}=0$. Inserting finally (2.18) into (2.6), (2.7) one obtains for the Green function (2.13)

$$
\begin{aligned}
& M^{6} G^{a_{1} a_{2} a_{3} a_{4}}\left(k_{1}, k_{2}, k_{3}, k_{4}\right) \\
& =\sum_{\underline{k}, \underline{l} \neq 0, \underline{m} \underline{s \in \mathcal{S}_{4}}} \sum_{k l m}^{a_{s 1} a_{s 2} a_{s 3} a_{s 4}}\left(k_{s 1}, k_{s 2}, k_{s 3}, k_{s 4}\right)+\sum_{s \in \mathcal{S}_{4}} \Omega^{a_{s 1} a_{s 2} a_{s 3} a_{s 4}}\left(k_{s 1}, k_{s 2}, k_{s 3}, k_{s 4}\right) .
\end{aligned}
$$

On general grounds one expects the vertex function to be real analytic. In particular there must also be terms involving the delta function in the $V_{\underline{k l m}}$ above which cancel those of the $\Omega$-term (we will demonstrate this explicitly in the computation of $V_{121}$ in appendix A). Thus, provided the coupling is well-defined (finite) at all, the result will be independent of the way the zero momentum limit is taken. It is therefore desirable to find a convenient limiting procedure that simplifies the computation. To this end we first observe that in (2.8) the $k_{j 1}$ and $k_{j 2}$ components enter asymmetrically. In particular as long as the intermediate state is not the vacuum (i.e. $\underline{l} \neq 0$ in the second formula, and recalling that we are assuming that none of the operators involved has a non-zero vacuum expectation value) one can put $k_{j 2}=0, j=1,2,3,4$. We now compute the 4 -point vertex function at zero momentum through the limiting procedure:

$$
G^{a_{1} a_{2} a_{3} a_{4}}(0,0,0,0)=\left.\lim _{\kappa_{j} \rightarrow 0} G^{a_{1} a_{2} a_{3} a_{4}}\left(k_{1}, k_{2}, k_{3}, k_{4}\right)\right|_{k_{j}=\left(M \operatorname{sh} \kappa_{j}, 0\right)}
$$

where $\sum_{j=1}^{4} \operatorname{sh} \kappa_{j}=0$, and the limit is taken such that

$$
\left|\kappa_{i}\right| \neq\left|\kappa_{j}\right| \text { for } i \neq j \text {, and }\left|\kappa_{i}-\kappa_{j}\right| \neq\left|\kappa_{k}-\kappa_{l}\right| \text { for distinct pairs . }
$$

In view of (2.19) it is clear that the limit prescription in (2.22), which we will use in the following, has just been designed such that the $\Omega$ term does not have to be considered in computation of the coupling.

Before embarking on further computations let us comment on a few structural issues. On physical grounds one expects the intrinsic coupling to be both finite (in a theory with a mass gap) and positive (for $\mathcal{N}>0$ ) when the interaction is repulsive. Mathematically however it is a quite challenging problem to actually prove this, whatever non-perturbative definition of the theory one adopts. In the context of constructive (lattice) QFT such results seem to be available only for a single phase $\Phi_{2}^{4}$ theory (see e.g [2] for a survey). In the present context we wish to define the theory strictly terms of its form factors. Mathematically speaking one should then try to prove in particular that the right hand 
side of (2.21) defines a real analytic function. For the dominant low particle contributions we demonstrate in appendix A explicitly that all non-analytic (e.g. distributional) terms indeed cancel out. We have not attempted to prove this in general, nor can we estimate the rate of convergence of the sums in (2.21) on general grounds. In all the examples considered later however the series appears to be rapidly convergent; the terms are alternating in sign and decrease in magnitude very quickly with increasing particle numbers.

\subsection{State parameterization}

Here we assume that the single particle spectrum consists only of an $\mathrm{O}(n)$ vector multiplet of mass $M$. The one particle states $|a, \alpha\rangle$ are thus specified by an internal "isospin" label $a$ and the rapidity $\alpha$ (i.e. the spatial momentum of the state is $p=M \operatorname{sh} \alpha$ ). The states are normalized according to

$$
\langle a, \alpha \mid b, \beta\rangle=4 \pi \delta_{a b} \delta(\alpha-\beta)
$$

The condensed notation for the sum over states now becomes

$$
\begin{aligned}
& \sum_{\underline{m}}|\underline{m}\rangle\langle\underline{m}|\longleftrightarrow| 0\rangle\langle 0|+\sum_{m=1}^{\infty} \sum_{a_{1}, \ldots a_{m}} \\
& \int_{-\infty}^{\infty} \frac{\mathrm{d} \theta_{1}}{4 \pi} \int_{-\infty}^{\theta_{1}} \frac{\mathrm{d} \theta_{2}}{4 \pi} \ldots \int_{-\infty}^{\theta_{m-1}} \frac{\mathrm{d} \theta_{m}}{4 \pi}\left|a_{1}, \theta_{1} ; \ldots ; a_{m}, \theta_{m}\right\rangle^{\text {in in }}\left\langle a_{1}, \theta_{1} ; \ldots ; a_{m}, \theta_{m}\right| .
\end{aligned}
$$

It is often convenient (for a fixed $m$ ) to perform the change of variables

$$
u_{j}=\theta_{j}-\theta_{j+1}, \quad j=1, \ldots, m-1, \quad \Lambda=\frac{1}{2} \ln \left(\frac{\sum_{j} e^{\theta_{j}}}{\sum_{j} e^{-\theta_{j}}}\right)
$$

since in terms of these variables the total energy and momentum of the states take on a simpler form:

$$
\left(E_{\underline{m}}, P_{\underline{m}}\right) \longleftrightarrow\left(M \sum_{j=1}^{m} \operatorname{ch} \theta_{j}, M \sum_{j=1}^{m} \operatorname{sh} \theta_{j}\right)=\left(M^{(m)}(u) \operatorname{ch} \Lambda, M^{(m)}(u) \operatorname{sh} \Lambda\right)
$$

where the eigenvalues $M_{\underline{m}}=\sqrt{E_{\underline{m}}^{2}-P_{\underline{m}}^{2}}$ of the mass operator are given by

$$
M^{(m)}(u)=M\left[m+2 \sum_{i<j} \operatorname{ch}\left(u_{i}+\ldots+u_{j-1}\right)\right]^{1 / 2} .
$$


Correspondingly the integration measures in (2.25) above are replaced by

$$
\int_{-\infty}^{\infty} \frac{\mathrm{d} \theta_{1}}{4 \pi} \int_{-\infty}^{\theta_{1}} \frac{\mathrm{d} \theta_{2}}{4 \pi} \ldots \int_{-\infty}^{\theta_{m-1}} \frac{\mathrm{d} \theta_{m}}{4 \pi} \longrightarrow \int_{0}^{\infty} \frac{\mathrm{d}^{m-1} u}{(4 \pi)^{m-1}} \int_{-\infty}^{\infty} \frac{\mathrm{d} \Lambda}{4 \pi}
$$

For later reference we also display the inverse transformation

$$
\begin{aligned}
& \theta_{j}=u_{j}+\ldots+u_{m-1}+u_{m}+\Lambda, \quad j=1, \ldots, m, \\
& \text { where } \quad u_{m}:=\frac{1}{2} \ln \left(\frac{1+\sum_{j=1}^{m-1} e^{-u_{j}-\ldots-u_{m-1}}}{1+\sum_{j=1}^{m-1} e^{u_{j}+\ldots+u_{m-1}}}\right) .
\end{aligned}
$$

\subsection{The two point function}

The spectral function (2.17) appearing in the representation of the two point function can be written as a sum of contributions of fixed particle number $m$

$$
\rho(\mu)=\sum_{0<m \text { odd }} \rho^{(m)}(\mu)
$$

where only odd numbers of particles contribute due to our assumption that the fields $\Phi^{a}$ are parity odd. We normalize the fields $\Phi^{a}$ by

$$
\left\langle 0\left|\Phi^{a}(0)\right| b, \alpha\right\rangle=\delta_{b}^{a},
$$

rendering the 1-particle contribution to the spectral density simply

$$
\rho^{(1)}(\mu)=\delta(\mu-M)
$$

The $m \geq 3$-particle contribution to the spin spectral function (2.17) is given by

$$
\rho^{(m)}(\mu)=\int_{0}^{\infty} \frac{\mathrm{d}^{m-1} u}{(4 \pi)^{m-1}} \delta\left(\mu-M^{(m)}(u)\right) I_{m}(u),
$$

with

$$
I_{m}(u):=\frac{1}{n} \sum_{a} \sum_{a_{1}, \ldots, a_{m}}\left|\mathcal{F}_{a_{1} \ldots a_{m}}^{a}\left(\theta_{1}, \ldots, \theta_{m}\right)\right|^{2}
$$


which equals $I_{m}^{11}(\theta)$ under the integral. The function $\mathcal{F}^{a}$ featuring here corresponds to the matrix element of $\Phi^{a}$ between vacuum and an $m$-particle in-state as in (2.9)

$$
\mathcal{F}_{a_{1} \ldots a_{m}}^{a}\left(\theta_{1}, \ldots, \theta_{m}\right)=\left\langle 0\left|\Phi^{a}(0)\right| a_{1}, \theta_{1} ; \ldots ; a_{m}, \theta_{m}\right\rangle^{\text {in }}, \quad \theta_{m}<\ldots<\theta_{1}
$$

The inverse 2-point function has a low momentum expansion of the form

$$
G(k)^{-1}=Z_{\mathrm{R}}^{-1}\left[M_{\mathrm{R}}^{2}+k^{2}+O\left(k^{4}\right)\right]
$$

with

$$
M_{\mathrm{R}}^{2}=M^{2} \frac{\gamma_{2}}{\delta_{2}}, \quad Z_{\mathrm{R}}=\frac{\gamma_{2}^{2}}{\delta_{2}},
$$

where $\gamma_{2}, \delta_{2}$ are spectral moments:

$$
\gamma_{2}=M^{2} \int_{0}^{\infty} \frac{\mathrm{d} \mu}{\mu^{2}} \rho(\mu), \quad \delta_{2}=M^{4} \int_{0}^{\infty} \frac{\mathrm{d} \mu}{\mu^{4}} \rho(\mu)
$$

\subsection{The intrinsic coupling revisited}

In (2.15) we left open the choice of the normalization constant $\mathcal{N}$ because for different models different choices are convenient. In analytical and numerical lattice computations (at fixed cutoff) it is often easier to compute the second moment mass $M_{\mathrm{R}}$ instead of the (exponential) spectral mass $M$ (in lattice units). For ease of comparison with these techniques we thus choose $\mathcal{N}=M_{\mathrm{R}}^{2} / M^{2}$, i.e. we define the intrinsic coupling by

$$
g_{\mathrm{R}}=-\frac{M_{\mathrm{R}}^{2}}{G(0)^{2}} \frac{1}{n^{2}} \sum_{a, b} G^{a a b b}(0,0,0,0) .
$$

Using $\mathrm{O}(n)$ symmetry it follows

$$
G^{a_{1} a_{2} a_{3} a_{4}}(0,0,0,0)=M^{-6} \gamma_{4}\left(\delta^{a_{1} a_{2}} \delta^{a_{3} a_{4}}+\delta^{a_{1} a_{3}} \delta^{a_{2} a_{4}}+\delta^{a_{1} a_{4}} \delta^{a_{2} a_{3}}\right)
$$

and hence we can write $(2.40)$ as

$$
g_{\mathrm{R}}=-\frac{n+2}{n} \frac{\gamma_{4}}{\gamma_{2} \delta_{2}}
$$


These spectral moments have, corresponding to the decomposition (2.31), an expansion in contributions arising from states with a fixed (odd) number of particles

$$
\gamma_{2}=1+\sum_{3 \geq m \text { odd }} \gamma_{2 ; m}, \quad \delta_{2}=1+\sum_{3 \geq m \text { odd }} \delta_{2 ; m}
$$

Similarly, corresponding to the sum in (2.21) we have

$$
\gamma_{4}=\sum_{k, l>0, m} \gamma_{4 ; k l m}, \quad \gamma_{4 ; k l m}=\gamma_{4 ; m l k}
$$

where the sum goes over odd integers $k, m$ and positive even integers $l$. To avoid writing many $\mathrm{O}(n)$ indices we will use

$$
\gamma_{4 ; k l m}=\frac{1}{3} \operatorname{Lim}_{\kappa_{j} \rightarrow 0} \sum_{s \in \mathcal{S}_{4}} v_{k l m}\left(\kappa_{s 1}, \kappa_{s 2}, \kappa_{s 3}, \kappa_{s 4}\right)
$$

where

$$
v_{k l m}\left(\kappa_{1}, \kappa_{2}, \kappa_{3}, \kappa_{4}\right)=\left.V_{k l m}^{1111}\left(k_{1}, k_{2}, k_{3}, k_{4}\right)\right|_{k_{j}=\left(M \operatorname{sh} \kappa_{j}, 0\right)},
$$

and the symbol Lim above means taking the limit $\kappa_{j} \rightarrow 0$ with the $\kappa_{j}$ satisfying $\sum_{j} \operatorname{sh} \kappa_{j}=$ 0 and the constraints in Eq. (2.23). 


\section{The nonlinear $\mathrm{O}(n)$ sigma-models}

As outlined in the introduction the form factor bootstrap (FFB) construction of an integrable quantum field theory starts from postulates of the on shell properties of the theory. By integrable here it is meant that the theory has an infinite set of conserved charges which entail that there is no particle production. This property usually is characteristic of non-relativistic quantum mechanics, remarkably here it holds for relativistic quantum field theories (QFTs) (assuming that the FFB approach does indeed define a QFT). In 4-dimensions absence of particle production implies that the theory is free but in two dimensions this is not so. In addition to the absence of particle production, one postulates the spectrum of stable particle states and their 2-particle S-matrix which has to satisfy the so-called Yang-Baxter (or factorization) equation (A.5).

In principle one could proceed without reference to a Lagrangian, but often contact to a Lagrangian description is desirable. Thus typically postulates of specific S-matrices are motivated by studies of associated Lagrangian QFTs. Unfortunately in most cases one cannot solve the QFTs to the extent necessary to really derive the candidate S-matrix, rather one has patches of partial information. This is in particular the case for the $\mathrm{O}(n)$ nonlinear sigma models formally described by a set of spin fields $\sigma^{a}, \quad a=1 \ldots n \geq 2$, with the constraint $\sigma^{2}=1$ and Lagrangian density $\propto\left(\partial_{\mu} \sigma\right)^{2}$. There is a wealth of information on these models which will be recalled when we study the various cases in the following sections, and for an overview we refer the reader to our previous paper [9]. In particular the spectrum of stable particles is thought to consist of an $\mathrm{O}(n)$ vector multiplet of mass $M$ without further bound states (i.e of the form of the spectrum considered in subsection 2.3). The S-matrix element (for $n \geq 2$ ) has the decomposition

$$
S_{a b ; c d}(\theta)=\sigma_{1}(\theta) \delta_{a b} \delta_{c d}+\sigma_{2}(\theta) \delta_{a c} \delta_{b d}+\sigma_{3}(\theta) \delta_{a d} \delta_{b c}
$$

where the center of mass energy is given by $\sqrt{s}=2 M \operatorname{ch} \theta / 2$.

Classically the theories have an infinite set of local and non-local conserved charges. One can argue that there are no anomalies which obstruct the existence of such charges in the quantum theory. In the case of the non-local charges for $n \geq 3$ the construction of Lüscher [17] is closely connected to the usual perturbative renormalizability and the (perturbative) asymptotic freedom of the model. Knowledge of the action of the non-local charges on the asymptotic states then restricts the S-matrix to the form postulated by 
Zamolodchikov and Zamolodchikov [3] for $n \geq 3$

$$
\begin{aligned}
& \sigma_{1}(\theta)=\frac{-2 \pi i \theta}{(i \pi-\theta)} \cdot \frac{s_{2}(\theta)}{(n-2) \theta-2 \pi i}, \\
& \sigma_{2}(\theta)=(n-2) \theta \cdot \frac{s_{2}(\theta)}{(n-2) \theta-2 \pi i}, \\
& \sigma_{3}(\theta)=-2 \pi i \cdot \frac{s_{2}(\theta)}{(n-2) \theta-2 \pi i},
\end{aligned}
$$

i.e. the invariant amplitudes are all given in terms of one amplitude which we have chosen here to be the invariant amplitude $s_{2}(\theta)$ in the symmetric traceless ("isospin 2") channel. The amplitude $s_{2}(\theta)$ is off-hand determined only up to so called CDD factors, which were initially [3] fixed by selecting the solution with the minimal number of poles and zeros in the physical strip. This solution for $s_{2}(\theta)$ is given by

$$
s_{2}(\theta)=-\exp \left\{2 i \int_{0}^{\infty} \frac{d \omega}{\omega} \sin (\theta \omega) \tilde{K}_{n}(\omega)\right\}
$$

with

$$
\tilde{K}_{n}(\omega)=\frac{e^{-\pi \omega}+e^{-2 \pi \frac{\omega}{n-2}}}{1+e^{-\pi \omega}} .
$$

The proposed identification of (3.2) - (3.4) with the S-matrix of the $\mathrm{O}(n)$ sigma-model passes several non trivial tests. First, the leading terms of its large $n$-expansion coincide with those obtained in leading orders of a field theoretical large $n$ computation. Second, in the determination of the exact $M / \Lambda$ ratio a consistency condition arises when matching the results of a perturbative computation against that obtained via the thermodynamic Bethe ansatz [40]. This consistency condition is also sensitive to the CDD factor; the minimal bootstrap solution (3.2) - (3.4) passes the test.

We note that the above formulae have a smooth $n \rightarrow 2$ limit. A study of the possible relation of the so defined $\mathrm{FFB} \mathrm{O}(2)$ model to the continuum limit of the lattice XY model (from the massive phase) will be the topic of a future publication [14.

Further we remark that the S-matrix for the case $n=1$ (Ising model) can also be written in the form (3.1) by setting

$$
\sigma_{1}(\theta)=\sigma_{2}(\theta)=0, \quad \sigma_{3}(\theta)=-1, \quad n=1 .
$$

The representation (3.1) is of course redundant in this case, but it does allow us in the following to discuss all $n \geq 1$ simultaneously. For example in all cases we have an 
expansion at low energies of the form

$$
S_{a b ; c d}(\theta)=-\delta_{a d} \delta_{b c}+i \theta D_{a b ; c d}+\mathcal{O}\left(\theta^{2}\right)
$$

in sharp contrast to a weak perturbation of a free field theory.

Having all the on-shell information covers all the physical information on the theory one observes from scattering of the stable particles, but off shell information is being explored if the system is probed by external sources weakly coupled to local operators with given quantum numbers.

\subsection{Derivation of the leading term in the FF expansion of $g_{\mathrm{R}}$}

For the leading 1-2-1 particle contribution to $g_{\mathrm{R}}$ a general model-independent expression can be given in terms of the derivative the S-matrix and the 3-particle form factor. For notational reasons we restrict attention here to the $\mathrm{O}(n)$ models considered later in more detail. The extension to a general integrable QFT without bound states is described in appendix A. For the $\mathrm{O}(n)$ models the formula reads

$$
\gamma_{4 ; 121}=\left.4 i \sum_{j=1}^{3} \frac{\mathrm{d} \sigma_{j}(\theta)}{\mathrm{d} \theta}\right|_{\theta=0}+\frac{1}{8 \pi} \int_{0}^{\infty} \mathrm{d} u\left\{\frac{1}{\operatorname{ch}^{2} u} f_{c}(u) f_{c}(-u)-\frac{64}{u^{2}}\right\} .
$$

Here $f_{c}(\theta)$ is a particular version of the 3 -particle form factor $\mathcal{F}_{b c d}^{a}\left(\theta_{1}, \theta_{2}, \theta_{3}\right)$ of the local field $\Phi^{a}$, supposed to correspond to the renormalized spin field $\sigma_{\mathrm{R}}^{a}$ in a Lagrangian construction. Explicitly

$$
f_{c}(\theta):=\mathcal{F}_{1 c c}^{1}(i \pi, \theta,-\theta)
$$

In order to derive (3.7) consider first more generally the $(1, l, 1)$ contribution in (2.21) with $l \geq 2$. Using (2.8) and switching to the explicit notation introduced in subsection 2.3 one can perform the integrals over the rapidities of the ' 1 ' particles. Then one decomposes the rapidity measure for the intermediate ' $\underline{l}$ ' particle contribution according to (2.26). Using (2.29) the $\Lambda$ integration can be performed and by means of (2.30) one arrives at

$$
\begin{aligned}
v_{1 l 1}\left(\kappa_{1}, \kappa_{2}, \kappa_{3}, \kappa_{4}\right) & =\frac{\pi}{2} \frac{1}{\operatorname{ch}^{2} \kappa_{1} \operatorname{ch}^{2} \kappa_{4}} \frac{1}{l !} \int \frac{\mathrm{d}^{l} \theta}{(4 \pi)^{l}} \frac{\delta\left(\theta_{1}, \ldots, \theta_{l}, \kappa_{1}, \kappa_{2}\right)}{\sum_{j=1}^{l} \operatorname{ch} \theta_{j}} I_{1 l 1}\left(-\kappa_{1}|\theta| \kappa_{4}\right) \\
& =\frac{1}{8 \operatorname{ch}^{2} \kappa_{1} \operatorname{ch}^{2} \kappa_{4}} \int \frac{\mathrm{d}^{l-1} u}{(4 \pi)^{l-1}} \frac{1}{\operatorname{ch}^{2} \Lambda_{*}} \frac{M^{2}}{M^{(l)}(u)^{2}} I_{1 l 1}\left(-\kappa_{1}|\theta| \kappa_{4}\right),
\end{aligned}
$$


where $I_{1 l 1}\left(-\kappa_{1}|\theta| \kappa_{4}\right):=I_{1 l 1}^{1111}\left(-\kappa_{1}|\theta| \kappa_{4}\right)$ is a product of generalized form factors as in (2.10), (2.11). Explicitly the correspondence to the matrix elements is

$$
\begin{aligned}
I_{1 l 1}\left(-\kappa_{1}|\theta| \kappa_{4}\right)=\sum_{b_{1}, \ldots, b_{l}}\langle & \left.1,-\kappa_{1}\left|\Phi^{1}(0)\right| b_{1}, \theta_{1} ; \ldots ; b_{l}, \theta_{l}\right\rangle^{\text {in }} \\
& \times{ }^{\text {in }}\left\langle b_{1}, \theta_{1} ; \ldots ; b_{l}, \theta_{l}\left|\Phi^{1}(0)\right| 1, \kappa_{4}\right\rangle .
\end{aligned}
$$

In the first expression we introduced the notation

$$
\delta\left(\theta_{1}, \ldots, \theta_{l}\right)=\delta\left(\sum_{j=1}^{l} \operatorname{sh} \theta_{j}\right)
$$

in the second one $\Lambda_{*}$ is defined by

$$
\operatorname{sh} \Lambda_{*}=-\frac{M}{M^{(l)}(u)}\left(\operatorname{sh} \kappa_{1}+\operatorname{sh} \kappa_{2}\right) .
$$

As remarked before the generalized form factors can be expressed in terms of form factors of the same operator and delta distributions by an explicit combinatorial formula. We shall usually just display the specific version needed. A discussion of the general formula can be found in the appendix of [16]. For the generalized form factor entering (3.10) the formula reads

$$
\begin{aligned}
& \left.\left\langle a_{1},-\kappa_{1}\left|\Phi^{a_{2}}(0)\right| b_{1}, \theta_{1} ; b_{2}, \theta_{2}\right\rangle\right\rangle\left.^{\text {in }}\right|_{\theta_{1}>\theta_{2}}=\mathcal{F}_{a_{1} b_{1} b_{2}}^{a_{2}}\left(-\kappa_{1}+i \pi-i \epsilon, \theta_{1}, \theta_{2}\right) \\
& +4 \pi \delta_{a_{1} b_{1}} \delta_{a_{2} b_{2}} \delta\left(\kappa_{1}+\theta_{1}\right)+4 \pi S_{b_{1} b_{2} ; a_{2} a_{1}}\left(\theta_{1}-\theta_{2}\right) \delta\left(\kappa_{1}+\theta_{2}\right) .
\end{aligned}
$$

Substituting this in Eq. (3.9) we obtain

$$
\begin{aligned}
& v_{121}=\frac{1}{64 \pi \operatorname{ch}^{2} \kappa_{1} \operatorname{ch}^{2} \kappa_{4}} \cdot\left\{\int_{-\infty}^{\infty} \mathrm{d} \alpha_{1} \frac{1}{\operatorname{ch} \bar{\alpha}_{2}\left(\operatorname{ch} \alpha_{1}+\operatorname{ch} \bar{\alpha}_{2}\right)}\right. \\
& \times \mathcal{F}_{1 x y}^{1}\left(i \pi-\kappa_{1}-i \epsilon, \alpha_{1}, \bar{\alpha}_{2}\right) \mathcal{F}_{1 x y}^{1}\left(i \pi-\kappa_{4}-i \epsilon,-\alpha_{1},-\bar{\alpha}_{2}\right) \\
& +\frac{8 \pi}{\operatorname{ch} \kappa_{3}\left(\operatorname{ch} \kappa_{3}+\operatorname{ch} \kappa_{4}\right)} \mathcal{F}_{111}^{1}\left(i \pi-\kappa_{1}-i \epsilon, \kappa_{4}, \kappa_{3}\right) \\
& \left.+\frac{8 \pi}{\operatorname{ch} \kappa_{2}\left(\operatorname{ch} \kappa_{1}+\operatorname{ch} \kappa_{2}\right)} \mathcal{F}_{111}^{1}\left(i \pi-\kappa_{4}-i \epsilon, \kappa_{1}, \kappa_{2}\right)\right\}
\end{aligned}
$$

Here we used the simplifications discussed above and the real analyticity property (3.16e) below. Moreover, we changed the integration variable from the difference of the two rapidities to one of the rapidities $\left(\alpha_{1}\right)$. The other rapidity $\left(\bar{\alpha}_{2}\right)$ is then the solution of the transcendental equation

$$
\operatorname{sh} \alpha_{1}+\operatorname{sh} \bar{\alpha}_{2}+\operatorname{sh} \kappa_{1}+\operatorname{sh} \kappa_{2}=0
$$


and is an analytic function of $\alpha_{1}$. There are no contributions from terms involving deltafunctions such as $\delta\left(\kappa_{1}+\kappa_{4}\right)$ appearing in $I_{121}$ since we are taking the limit (2.22) where these delta-functions vanish. (These terms are however crucial to cancel corresponding singularities in the $\Omega$ term; c.f. appendix A).

The form factors appearing in (3.14) obey a system of functional equations which allow one to further simplify the expression. Let us recall these equations in the form relevant to the three-particle form factor $\mathcal{F}_{a b c}^{d}(\alpha, \beta, \gamma)$ in the $\mathrm{O}(n)$ model.

$$
\begin{aligned}
\mathcal{F}_{a b c}^{d}(\alpha, \beta, \gamma) & =S_{b c ; y x}(\beta-\gamma) \mathcal{F}_{a x y}^{d}(\alpha, \gamma, \beta), \\
\mathcal{F}_{a b c}^{d}(\alpha, \beta, \gamma) & =\mathcal{F}_{c a b}^{d}(\gamma+2 \pi i, \alpha, \beta), \\
\mathcal{F}_{a b c}^{d}(\alpha, \beta, \gamma) & =\mathcal{F}_{a b c}^{d}(\alpha+\lambda, \beta+\lambda, \gamma+\lambda), \\
\mathcal{F}_{a b c}^{d}(\alpha, \beta, \gamma) & =\mathcal{F}_{c b a}^{d}(-\gamma,-\beta,-\alpha), \\
{\left[\mathcal{F}_{a b c}^{d}(\alpha, \beta, \gamma)\right]^{*} } & =\mathcal{F}_{a b c}^{d}\left(-\alpha^{*},-\beta^{*},-\gamma^{*}\right) .
\end{aligned}
$$

Here the S-matrix appearing in the exchange axiom (3.16a) is the $\mathrm{O}(n)$ S-matrix (3.1). (3.16d) and (3.16e) express the parity invariance and real analyticity property of the form factors, respectively. The homogeneous axioms (3.16) are supplemented by the inhomogeneous residue equation

$$
\lim _{\alpha \rightarrow \beta+i \pi}(\alpha-\beta-i \pi) \mathcal{F}_{a b c}^{d}(\alpha, \beta, \gamma)=2 i\left\{\delta_{a b} \delta_{c d}-S_{b c ; a d}(\beta-\gamma)\right\}
$$

We now take advantage of the analytic properties of the form factors and change the integration contour in (3.14) from the real axis to a curve $\mathcal{C}$ which is arbitrary except that it has to stay within the 'physical strip' $0<\operatorname{Im} \alpha_{1}<\pi / 2$. Along this contour we can put $\epsilon=0$ and also the limit $\kappa_{i} \rightarrow 0$ can safely be taken. The integrated part of (3.14) then simplifies to

$$
v_{121}^{(I I)}=\frac{1}{128 \pi} \int_{\mathcal{C}} \frac{\mathrm{d} \alpha}{\operatorname{ch}^{2} \alpha} f_{b}(\alpha) f_{b}(-\alpha),
$$

where we introduced the shorthands

$$
\begin{aligned}
& f_{a b c}^{d}(\alpha):=\mathcal{F}_{a b c}^{d}(i \pi, \alpha,-\alpha), \\
& f_{1 b c}^{1}(\alpha)=: \quad \delta_{b c} f_{b}(\alpha) \quad(\text { no sum) } .
\end{aligned}
$$


Of course, one has to take into account the contribution of those singular points of the integrand that get crossed when deforming the contour of integration. There are two such singular points:

$$
\alpha_{1}=\kappa_{4}+i \epsilon \quad \text { and } \quad \bar{\alpha}_{2}=-\kappa_{1}-i \epsilon
$$

which never coincide if (2.23) holds.

Applying Cauchy's theorem one can evaluate the contribution from the first singular point using the residue axiom (3.17). This gives

$$
-\frac{1}{16 \operatorname{ch}^{2} \kappa_{1} \operatorname{ch}^{2} \kappa_{4} \operatorname{ch} \bar{\alpha}_{2}\left(\operatorname{ch} \alpha_{1}+\operatorname{ch} \bar{\alpha}_{2}\right)}\left\{\mathcal{F}_{111}^{1}\left(i \pi-\kappa_{1}-i \epsilon, \alpha_{1}, \bar{\alpha}_{2}\right)-\mathcal{F}_{111}^{1}\left(i \pi-\kappa_{1}-i \epsilon, \bar{\alpha}_{2}, \alpha_{1}\right)\right\},
$$

where in the second term we also used the exchange axiom (3.16a). After taking the limit $\epsilon \rightarrow 0$, which is possible if (2.23) holds, the contribution of the first singular point becomes

$$
\frac{1}{16 \operatorname{ch}^{2} \kappa_{1} \operatorname{ch}^{2} \kappa_{4} \operatorname{ch} \kappa_{3}\left(\operatorname{ch} \kappa_{3}+\operatorname{ch} \kappa_{4}\right)}\left\{\mathcal{F}_{111}^{1}\left(i \pi-\kappa_{1}, \kappa_{3}, \kappa_{4}\right)-\mathcal{F}_{111}^{1}\left(i \pi-\kappa_{1}, \kappa_{4}, \kappa_{3}\right)\right\} .
$$

The contribution of the second singularity is similar:

$$
\frac{1}{16 \operatorname{ch}^{2} \kappa_{1} \operatorname{ch}^{2} \kappa_{4} \operatorname{ch} \kappa_{2}\left(\operatorname{ch} \kappa_{1}+\operatorname{ch} \kappa_{2}\right)}\left\{\mathcal{F}_{111}^{1}\left(i \pi-\kappa_{4}, \kappa_{2}, \kappa_{1}\right)-\mathcal{F}_{111}^{1}\left(i \pi-\kappa_{4}, \kappa_{1}, \kappa_{2}\right)\right\} .
$$

Putting together the contribution of the singular points and the last two terms of (3.14) the non-integrated contribution can be written as

$$
v_{121}^{(I)} \doteq \frac{1}{8 \operatorname{ch}^{2} \kappa_{1} \operatorname{ch}^{2} \kappa_{4} \operatorname{ch} \kappa_{3}\left(\operatorname{ch} \kappa_{3}+\operatorname{ch} \kappa_{4}\right)}\left\{\mathcal{F}_{111}^{1}\left(i \pi-\kappa_{1}, \kappa_{3}, \kappa_{4}\right)+\mathcal{F}_{111}^{1}\left(i \pi-\kappa_{1}, \kappa_{4}, \kappa_{3}\right)\right\}
$$

where $\doteq$ indicates equality after the symmetrization over the elements of the permutation group $\mathcal{S}_{4}$ has been carried out.

We now use the Smirnov axioms (3.16) and (3.17) to simplify the non-integrated part in the (symmetrized) $\kappa_{i} \rightarrow 0$ limit. It is convenient to first introduce the reduced form factor $\mathcal{G}_{a b c}^{d}(\alpha, \beta, \gamma)$ by

$$
\mathcal{F}_{a b c}^{d}(\alpha, \beta, \gamma)=T_{3}(\alpha, \beta, \gamma) \mathcal{G}_{a b c}^{d}(\alpha, \beta, \gamma)
$$

Here and in the following we set

$$
T_{N}\left(\theta_{1}, \ldots, \theta_{N}\right):=\prod_{1 \leq i<j \leq N} T\left(\theta_{i}-\theta_{j}\right)
$$


where $T$ is basically the tanh-function $T(\theta):=\tanh \theta / 2$. Note $T(\theta)$ has a simple pole at $\theta=i \pi, T(i \pi-\theta)=-T(i \pi+\theta)=-2 / \theta+\mathcal{O}(\theta)$, and a simple zero at $\theta=0$. The advantage of the representation (3.21) is that the singularities are carried by the tanh factors and the reduced form factor $\mathcal{G}_{a b c}^{d}$ is analytic everywhere in the physical strip. In particular, for small $\alpha, \beta$ and $\gamma$ it can be expanded as

$$
\mathcal{G}_{a b c}^{d}(i \pi+\alpha, \beta, \gamma)=J_{a b c}^{d}+(\alpha-\gamma) K_{a b c}^{d}+(\beta-\gamma) L_{a b c}^{d}+\ldots
$$

where the dots stand for terms higher order in $\alpha, \beta$ and $\gamma$. We can compute the constant tensors appearing in the expansion (3.23) using the form factors equations. From the residue axiom (3.17) we can immediately fix

$$
J_{a b c}^{d}=i\left(\delta_{a b} \delta_{c d}+\delta_{a c} \delta_{b d}\right), \quad K_{a b c}^{d}+L_{a b c}^{d}=D_{b c ; a d}
$$

To determine the expansion coefficients individually we employ the exchange relation (3.16a) and find

$$
K_{a b c}^{d}=D_{b c ; a d}-D_{b c ; d a}, \quad L_{a b c}^{d}=D_{b c ; d a} .
$$

Using the expansion (3.23), for small $\kappa$ the non-integrated contribution becomes

$$
v_{121}^{(I)} \doteq \frac{1}{4} \frac{\kappa_{3}-\kappa_{4}}{\left(\kappa_{1}+\kappa_{3}\right)\left(\kappa_{1}+\kappa_{4}\right)}\left\{2 i+\left(\kappa_{3}-\kappa_{4}\right) D+O\left(\kappa^{2}\right)\right\}
$$

where

$$
D=D_{11 ; 11}=-\left.i \sum_{j=1}^{3} \frac{\mathrm{d} \sigma_{j}(\theta)}{\mathrm{d} \theta}\right|_{\theta=0} .
$$

This can be simplified by noting that upon averaging over the permutations

$$
\frac{1}{\kappa_{1}+\kappa_{4}} \doteq \frac{1}{\kappa_{1}+\kappa_{3}} \doteq 0
$$

and similarly

$$
\frac{\kappa_{4}}{\kappa_{1}+\kappa_{4}} \doteq \frac{\kappa_{3}}{\kappa_{1}+\kappa_{3}} \doteq-\frac{\kappa_{3}}{\kappa_{1}+\kappa_{4}} \doteq-\frac{\kappa_{4}}{\kappa_{1}+\kappa_{3}} \doteq \frac{1}{2} .
$$

After this simplification we have for the non-integrated contribution

$$
v_{121}^{(I)} \doteq-\frac{1}{2} D
$$


and hence the non-integrated part of the leading contribution to the four-point coupling eventually becomes

$$
\gamma_{4 ; 121}^{(I)}=-4 D
$$

For the Ising model the S-matrix is constant and therefore $\gamma_{4 ; 121}^{(I)}=0$ for $n=1$. For $n \geq 2$ we use (3.3) and find

$$
\gamma_{4 ; 121}^{(I)}=-\frac{4}{\pi}+8 \int_{0}^{\infty} \mathrm{d} \omega \tilde{K}_{n}(\omega)
$$

The integrated part (3.18) (which is in this form rather useful for numerical evaluation) can be written in an alternative form using the the residue axiom which implies

$$
f_{a b c}^{d}(\theta)=-\frac{4}{\theta} J_{a b c}^{d}+\mathcal{O}(1)=-\frac{4 i}{\theta}\left(\delta_{a b} \delta_{c d}+\delta_{a c} \delta_{b d}\right)+O(1)
$$

Using this we can explicitly subtract the singular part in (3.18) and shift the contour back to the real axis. Noting also that the integrand is an even function of $\alpha$ we arrive at

$$
\gamma_{4 ; 121}^{(I I)}=\frac{1}{8 \pi} \int_{0}^{\infty} \mathrm{d} u\left\{\frac{1}{\operatorname{ch}^{2} u} f_{b}(u) f_{b}(-u)-\frac{64}{u^{2}}\right\} .
$$

The extension of the formula (3.7) to general integrable models without bound states is described in appendix A.

\subsection{The three particle form factor}

Only the special three-particle form factor $f_{a b c}^{d}(\theta)$ in (3.19) is necessary to compute the leading contribution (3.7) to $g_{\mathrm{R}}$. It turns out to obey an autonomous system of functional equations (in a single variable) that derives from the form factor equations satisfied by $\mathcal{F}_{a b c}^{d}(\alpha, \beta, \gamma)$. Solving it allows one to compute $f_{b}(\theta)$ - and hence to evaluate (3.7) - in situations where the general form factors are not known.

We begin by noting that the functions $f_{a b c}^{d}(\theta)$ are real analytic, i.e. $\left[f_{a b c}^{d}(\theta)\right]^{*}=f_{a b c}^{d}\left(-\theta^{*}\right)$, in the physical strip $0 \leq \operatorname{Im} \theta \leq \pi$, with simple poles at $\theta=0$ and $\theta=\frac{i \pi}{2}$. Moreover, using (3.16) b,d) one can easily deduce that it is symmetric in its last two indices,

$$
f_{a b c}^{d}(\theta)=f_{a c b}^{d}(\theta)
$$

Using (3.16a) one obtains

$$
f_{a b c}^{d}(\theta)=S_{b c ; y x}(2 \theta) f_{a x y}^{d}(-\theta)
$$


and finally combining (3.16a-d) results in

$$
f_{a b c}^{d}(i \pi-\theta)=S_{c a ; y x}(\theta) S_{y b ; z l}(2 \theta) S_{l x ; v w}(\theta) f_{w v z}^{d}(i \pi+\theta)
$$

These are the consequences of the homogeneous form factor axioms; they are supplemented by the residue equations

$$
\begin{aligned}
\operatorname{Res} f_{a b c}^{d}(0) & =-4 i\left(\delta_{a b} \delta_{c d}+\delta_{a c} \delta_{b d}\right), \\
\operatorname{Res} f_{a b c}^{d}\left(\frac{i \pi}{2}\right) & =i\left\{\delta_{b c} \delta_{a d}-S_{c a ; b d}\left(\frac{i \pi}{2}\right)\right\} .
\end{aligned}
$$

In view of Eq. (3.35) we can parameterize $f$ as

$$
f_{a b c}^{d}(\theta)=k(\theta) \delta_{a d} \delta_{b c}+l(\theta)\left[\delta_{a c} \delta_{b d}+\delta_{a b} \delta_{c d}\right]
$$

Then the contribution $\gamma_{4 ; 121}^{(I I)}$ is given by

$$
\gamma_{4 ; 121}^{(I I)}=\frac{1}{8 \pi} \int_{0}^{\infty} \mathrm{d} u\left\{\frac{n k(u) k(-u)+2 k(u) l(-u)+2 k(-u) l(u)+4 l(u) l(-u)}{\operatorname{ch}^{2} u}-\frac{64}{u^{2}}\right\} .
$$

In terms of the two functions $k(\theta)$ and $l(\theta)$ Eq. (3.36) can be written as

$$
\begin{aligned}
k(\theta) & =\left[s_{2}(2 \theta)+n \sigma_{1}(2 \theta)\right] k(-\theta)+2 \sigma_{1}(2 \theta) l(-\theta), \\
l(\theta) & =s_{2}(2 \theta) l(-\theta),
\end{aligned}
$$

while (3.37) becomes

$$
\begin{aligned}
k(i \pi-\theta) & =\left[A_{11}(\theta) k(i \pi+\theta)+A_{12}(\theta) l(i \pi+\theta)\right] a(\theta) s_{2}(\theta)^{2} s_{2}(2 \theta) \\
l(i \pi-\theta) & =\left[A_{21}(\theta) k(i \pi+\theta)+A_{22}(\theta) l(i \pi+\theta)\right] a(\theta) s_{2}(\theta)^{2} s_{2}(2 \theta) .
\end{aligned}
$$

Here

$$
a(\theta)=\frac{(n-2) \theta+2 i \pi}{(i \pi-\theta)(i \pi-2 \theta)[(n-2) \theta-i \pi][(n-2) \theta-2 i \pi]^{2}},
$$

and

$$
\begin{aligned}
& A_{11}(\theta)=(\theta-i \pi)\left[2(n-2)^{2} \theta^{3}+(n-2)(n-4) \theta^{2} i \pi+(n+2) \theta \pi^{2}-2 i \pi^{3}\right] \\
& A_{12}(\theta)=-4(n-2) i \pi \theta(\theta-i \pi)(\theta+i \pi) \\
& A_{21}(\theta)=-2(n-4) i \pi^{3} \theta \\
& A_{22}(\theta)=A_{11}(-\theta)
\end{aligned}
$$


The matrix $A(\theta)$ satisfies

$$
A(\theta)^{-1}=A(-\theta) a(\theta) a(-\theta), \quad \operatorname{det} A(\theta)=\frac{1}{a(\theta) a(-\theta)}
$$

The functional equations (3.42), (3.43) still contain the transcendental function $s_{2}(\theta)$. It can be eliminated by the following standard procedure. We introduce the function $u(\theta)$ as the unique solution of

$$
\begin{aligned}
u(\theta) & =s_{2}(\theta) u(-\theta), \\
u(i \pi-\theta) & =-u(i \pi+\theta),
\end{aligned}
$$

subject to the normalization condition

$$
u(i \pi-\theta)=\frac{1}{\theta}+\mathcal{O}(\theta)
$$

Using the results of Appendix D one can immediately write down the solution

$$
u(\theta)=-\frac{1}{2} T(\theta) e^{\Delta(\theta)},
$$

where in (D.3) of course the kernel $\tilde{K}_{n}(\omega)$ defined in (3.4) has to be used. Introducing

$$
Y(\theta)=\frac{2 i}{u_{0}} u(i \pi-\theta) u(i \pi+\theta) u(2 \theta), \quad u_{0}=u^{\prime}(0)=-\frac{1}{4} e^{\Delta(0)} .
$$

we parameterize $k, l$ as

$$
k(\theta)=Y(\theta) K(\theta) \quad \text { and } \quad l(\theta)=Y(\theta) L(\theta) .
$$

Rewriting then the functional equations (3.42), (3.43) in terms of $K$ and $L$, the new system involves only rational coefficient functions. Explicitly they read

$$
\begin{aligned}
K(\theta) & =\frac{-1}{[(n-2) \theta-i \pi](i \pi-2 \theta)}\{[(n-2) \theta+i \pi](i \pi+2 \theta) K(-\theta)+4 i \pi \theta L(-\theta)\} \\
L(\theta) & =L(-\theta)
\end{aligned}
$$

and

$$
\begin{aligned}
K(i \pi-\theta) & =\left[A_{11}(\theta) K(i \pi+\theta)+A_{12}(\theta) L(i \pi+\theta)\right] a(\theta) \\
L(i \pi-\theta) & =\left[A_{21}(\theta) K(i \pi+\theta)+A_{22}(\theta) L(i \pi+\theta)\right] a(\theta)
\end{aligned}
$$


respectively. The first equation of (3.53) can be used to eliminate $L(\theta)$ in favor of $K(\theta)$ via

$$
L(\theta)=\frac{1}{4 i \pi \theta}\{[i \pi-(n-2) \theta](i \pi-2 \theta) K(\theta)-[i \pi+(n-2) \theta](i \pi+2 \theta) K(-\theta)\},
$$

and (3.55) also solves the second equation of (3.53). Inserting (3.55) into (3.54) results in a single linear functional equation for $K(\theta)$. The normalization of the solution is fixed by the residue equations (3.39).

We expect that this procedure can be used to compute $f_{b}(\theta)$ and hence the leading contribution to the coupling for all $\mathrm{O}(n)$ models. For the $\mathrm{O}(2)$ model we demonstrate this in section 6 .

\subsection{Sub-leading contributions}

In order to achieve higher accuracy and to obtain some clue on the rate of convergence of the series (2.44) we will compute some of the sub-leading terms as well. It turns out that the 1-2-1 term indeed gives the numerically most important contribution to the coupling. But based on the computation of the sub-leading terms the numerical result can also be endowed with an intrinsic error estimate. Our results indicate that the next important contributions to the coupling are $(1,2,3)+(3,2,1)$ and $(1,4,1)$. Its explicit evaluation is deferred to appendices $\mathrm{C}$ and $\mathrm{B}$. The difficulty in the evaluation lies in the rapidly varying nature of the integrands, which have in the multidimensional phase space many zeros and (integrable) singularities. To deal with these we have either decomposed the integrand into appropriate parts or avoided the singularities by shifting some contours of integration into the complex plane.

The $(1,4,1)$ contribution is the $l=4$ case of Eq. (3.9) and will be evaluated in appendix B. Here we prepare the ground for the evaluation of the $(1,2,3)+(3,2,1)$ terms. More generally let us examine the $(1,2, m)+(m, 2,1)$ contribution and to this end return to (2.8). Performing the internal rapidity integrations one obtains

$$
\begin{array}{r}
v_{12 m}\left(\kappa_{1}, \kappa_{2}, \kappa_{3}, \kappa_{4}\right)=\frac{\pi^{2}}{\operatorname{ch}^{2} \kappa_{1}} \frac{1}{m !} \int \frac{\mathrm{d} \xi_{1} \mathrm{~d} \xi_{2}}{(4 \pi)^{2}} \frac{\delta\left(\xi_{1}, \xi_{2}, \kappa_{1}, \kappa_{2}\right)}{\operatorname{ch} \xi_{1}+\operatorname{ch} \xi_{2}} \\
\times \int \frac{\mathrm{d}^{m} \theta}{(4 \pi)^{m}} \frac{\delta\left(\theta_{1}, \ldots, \theta_{m},-\kappa_{4}\right)}{\sum_{j=1}^{m} \operatorname{ch} \theta_{j}} I_{12 m}\left(-\kappa_{1}\left|\xi_{2}, \xi_{1}\right| \theta\right) .
\end{array}
$$

Next one spells out $I_{12 m}:=I_{12 m}^{1111}$ by inserting the formula expressing the generalized form factors in terms of ordinary form factors. Taking advantage of the S-matrix exchange 
relations many of terms contribute equally upon integration and one ends up with four terms

$$
v_{12 m}=v_{12 m}^{(I)}+v_{12 m}^{(I I)}+v_{12 m}^{(I I I)}+v_{12 m}^{(I V)}
$$

with

$$
\begin{aligned}
& v_{12 m}^{(I)}\left(\kappa_{1}, \kappa_{2}, \kappa_{3}, \kappa_{4}\right) \sim \frac{1}{16(4 \pi)^{m} m !} \sum_{b_{1}, b_{2}} \sum_{a_{1}, \ldots, a_{m}} \int \mathrm{d}^{2} \beta \frac{\delta\left(\beta_{1}, \beta_{2}, \kappa_{1}, \kappa_{2}\right)}{\operatorname{ch} \beta_{1}+\operatorname{ch} \beta_{2}} \\
& \int \mathrm{d}^{m} \alpha \frac{\delta\left(\alpha_{1}, \ldots, \alpha_{m},-\kappa_{4}\right)}{\sum_{i=1}^{k} \operatorname{ch} \alpha_{i}} \mathcal{F}_{1 b_{1} b_{2}}^{1}\left(-\kappa_{1}+i \pi_{-}, \beta_{1}, \beta_{2}\right) \\
& \mathcal{F}_{b_{2} b_{1} a_{1} a_{2} \ldots a_{m}}^{1}\left(\beta_{2}+i \pi_{-}, \beta_{1}+i \pi_{-}, \alpha_{1}, \ldots, \alpha_{m}\right) \mathcal{F}_{a_{1} a_{2} \ldots a_{m}}^{1}\left(\alpha_{1}, \ldots, \alpha_{m}\right)^{*}, \\
& v_{12 m}^{(I I)}\left(\kappa_{1}, \kappa_{2}, \kappa_{3}, \kappa_{4}\right) \sim \frac{1}{8(4 \pi)^{m-1}(m-1) !} \sum_{b_{1}, b_{2}} \sum_{a_{2}, \ldots, a_{m}} \int \mathrm{d}^{2} \beta \frac{\delta\left(\beta_{1}, \beta_{2}, \kappa_{1}, \kappa_{2}\right)}{\operatorname{ch} \beta_{1}+\operatorname{ch} \beta_{2}} \\
& \int \mathrm{d}^{m-1} \alpha \frac{\delta\left(\beta_{1}, \alpha_{2} \ldots, \alpha_{m},-\kappa_{4}\right)}{\operatorname{ch} \beta_{1}+\sum_{i=2}^{m} \operatorname{ch} \alpha_{i}} \mathcal{F}_{1 b_{1} b_{2}}^{1}\left(-\kappa_{1}+i \pi_{-}, \beta_{1}, \beta_{2}\right) \\
& \mathcal{F}_{b_{2} a_{2} \ldots a_{m}}^{1}\left(\beta_{2}+i \pi_{-}, \alpha_{2}, \ldots, \alpha_{m}\right) \mathcal{F}_{b_{1} a_{2} \ldots a_{m}}^{1}\left(\beta_{1}, \alpha_{2} \ldots, \alpha_{m}\right)^{*}, \\
& v_{12 m}^{(I I I)}\left(\kappa_{1}, \kappa_{2}, \kappa_{3}, \kappa_{4}\right) \sim \frac{1}{16(4 \pi)^{m-2}(m-2) !} \sum_{b_{1}, b_{2}} \sum_{a_{3}, \ldots, a_{m}} \int \mathrm{d}^{2} \beta \frac{\delta\left(\beta_{1}, \beta_{2}, \kappa_{1}, \kappa_{2}\right)}{\operatorname{ch} \beta_{1}+\operatorname{ch} \beta_{2}} \\
& \int \mathrm{d}^{m-2} \alpha \frac{\delta\left(\alpha_{3}, \ldots, \alpha_{m}, \kappa_{3}\right)}{\operatorname{ch} \beta_{1}+\operatorname{ch} \beta_{2}+\sum_{i=3}^{m} \operatorname{ch} \alpha_{i}} \mathcal{F}_{1 b_{1} b_{2}}^{1}\left(-\kappa_{1}+i \pi_{-}, \beta_{1}, \beta_{2}\right) \\
& \mathcal{F}_{a_{3} \ldots a_{m}}^{1}\left(\alpha_{3}, \ldots, \alpha_{m}\right) \mathcal{F}_{b_{1} b_{2} a_{3} \ldots a_{m}}^{1}\left(\beta_{1}, \beta_{2}, \alpha_{3}, \ldots, \alpha_{m}\right)^{*}, \\
& v_{12 m}^{(I V)}\left(\kappa_{1}, \kappa_{2}, \kappa_{3}, \kappa_{4}\right) \sim \frac{1}{16(4 \pi)^{m-1} m !} \sum_{a_{1}, \ldots, a_{m}} \int \mathrm{d}^{m} \alpha \frac{\delta\left(\alpha_{1}, \ldots, \alpha_{m},-\kappa_{4}\right)}{\sum_{i=1}^{m} \operatorname{ch} \alpha_{i}} \\
& \mathcal{F}_{11 a_{1} a_{2} \ldots a_{m}}^{1}\left(-\kappa_{2}+i \pi_{-},-\kappa_{1}+i \pi_{-}, \alpha_{1}, \ldots, \alpha_{m}\right) \mathcal{F}_{a_{1} a_{2} \ldots a_{m}}^{1}\left(\alpha_{1}, \ldots, \alpha_{m}\right)^{*},
\end{aligned}
$$

where $\pi_{-}$stands for $\pi-\epsilon$. All integrals range from $-\infty$ to $+\infty$.

Further details of the computation of the 1-2-3 contribution are given in Appendix C. Note that for the numerical evaluation of the $k+l+m=6$ contributions we need the analytic expressions for the 5-particle form factor of the spin operator. Unfortunately these are at present only known for $n=1$ and $n=3$. For the Ising model all the form factors are explicitly known and in this case we have also computed the $k+l+m=8$ contributions. After a preparatory next section where we discuss the definition and measurement of the intrinsic coupling in the lattice regularization, we will discuss the cases $n=1,2,3$ in turn. 


\section{Lattice computations of $g_{\mathrm{R}}$}

In the subsequent sections we will compare the results of the form factor bootstrap coupling $g_{\mathrm{R}}$ with those obtained from the lattice theory. As noted earlier, in the framework of the lattice regularization there are two methods to compute $g_{\mathrm{R}}$ in the $\mathrm{O}(n)$ models: high temperature (= strong coupling) expansions and Monte Carlo simulations. Both approaches usually take the standard lattice action on a square lattice

$$
S=-\beta \sum_{x, \mu} \sigma(x) \cdot \sigma(x+\hat{\mu}),
$$

as the starting point, where $\sigma(x) \cdot \sigma(x)=\sum_{a} \sigma^{a}(x) \sigma^{a}(x)=1$.

The lattice definition of $g_{\mathrm{R}}(\beta)$ is as in Eq. (2.40)

$$
g_{\mathrm{R}}(\beta)=-\frac{1}{\xi_{2}^{2} G_{2}(0)^{2}} \frac{1}{n^{2}} \sum_{a, b} G_{4}^{a a b b},
$$

where all quantities are defined analogously to the continuum theory

$$
\begin{aligned}
G_{2}(k)= & \frac{1}{n} \sum_{a} \sum_{x} e^{i k x}\left\langle\sigma^{a}(x) \sigma^{a}(0)\right\rangle, \\
G_{4}^{a_{1} a_{2} a_{3} a_{4}}= & \sum_{x_{1}, x_{2}, x_{3}}\left\{\left\langle\sigma^{a_{1}}\left(x_{1}\right) \sigma^{a_{2}}\left(x_{2}\right) \sigma^{a_{3}}\left(x_{3}\right) \sigma^{a_{4}}(0)\right\rangle\right. \\
& \left.-\left[\left\langle\sigma^{a_{1}}\left(x_{1}\right) \sigma^{a_{2}}\left(x_{2}\right)\right\rangle\left\langle\sigma^{a_{3}}\left(x_{3}\right) \sigma^{a_{4}}(0)\right\rangle+2 \text { perms }\right]\right\},
\end{aligned}
$$

and $\xi_{2}$ is the second moment correlation length

$$
\xi_{2}^{2}=\frac{\mu_{2}}{4 G_{2}(0)}, \quad \mu_{2}=\frac{1}{n} \sum_{a} \sum_{x} x^{2}\left\langle\sigma^{a}(x) \sigma^{a}(0)\right\rangle .
$$

The coupling from the lattice regularization is defined as the continuum limit

$$
g_{\mathrm{R}}=\lim _{\beta \rightarrow \beta_{\mathrm{c}}} g_{\mathrm{R}}(\beta),
$$

where $\beta_{\mathrm{c}}$ is a critical point where the correlation length diverges (in lattice units).

Butera and Comi [12] have produced long high temperature series for $G_{2}(0), \mu_{2}$, and $G_{4}$ in the $\mathrm{O}(n)$ model with standard action, and Pelissetto and Vicari [13] have reanalyzed these 
series to compute estimates for the intrinsic coupling $g_{\mathrm{R}}$ for $n \leq 4$. Similar computations have been performed previously by Campostrini et al [11].

Our Monte Carlo simulations were of course done on a finite lattice, more precisely a square lattice of size $L$ (points) in each direction and periodic boundary conditions, both with the standard action (4.1) and the fixed point action of ref. [18]. The infinite volume lattice coupling $g_{\mathrm{R}}(\beta)$ is then obtained as the limit

$$
g_{\mathrm{R}}(\beta)=\lim _{L \rightarrow \infty} g_{\mathrm{R}}(\beta, L)
$$

of a finite volume coupling $g_{\mathrm{R}}(\beta, L)$ which is proportional to Binder's cumulant $u_{L}$ :

$$
\begin{aligned}
g_{\mathrm{R}}(\beta, L) & =\left(\frac{L}{\xi^{\mathrm{eff}}(\beta, L)}\right)^{2} u_{L}, \\
u_{L} & =1+\frac{2}{n}-\frac{\left\langle\left(\Sigma^{2}\right)^{2}\right\rangle}{\left\langle\Sigma^{2}\right\rangle^{2}},
\end{aligned}
$$

where $\Sigma^{a}=\sum_{x} \sigma^{a}(x)$. In this definition $\xi^{\text {eff }}(\beta, L)$ is an effective correlation length which converges to the second moment correlation length $\xi_{2}$ in the limit $L \rightarrow \infty$. In our computations we used the particular definition (as e.g. in ref. [7]):

$$
\xi^{\mathrm{eff}}(\beta, L)=\frac{1}{2 \sin (\pi / L)} \sqrt{\frac{G_{2}(0)}{G_{2}\left(k_{0}\right)}-1},
$$

where $k_{0}=(2 \pi / L, 0)$.

In our analysis of the Monte Carlo data we shall make the working assumption that one is allowed to replace the limiting procedure $\lim _{\beta \rightarrow \beta_{\mathrm{c}}} \lim _{L \rightarrow \infty}$ by

$$
\begin{aligned}
g_{\mathrm{R}} & =\lim _{z \rightarrow \infty} \hat{g}_{\mathrm{R}}(z), \quad z:=L / \xi^{\mathrm{eff}}(\beta, L), \\
\hat{g}_{\mathrm{R}}(z) & :=\lim _{\beta \rightarrow \beta_{\mathrm{c}}, z \text { fixed }} g_{\mathrm{R}}(\beta, L) .
\end{aligned}
$$

That is we attempt to first take the continuum limit at fixed physical volume and afterwards take the physical volume to infinity. The $z \rightarrow \infty$ limit of $\hat{g}_{\mathrm{R}}(z)$ is expected to be reached exponentially; for example in the leading order $1 / n$ expansion [10

$$
\hat{g}_{\mathrm{R}}(z)=\hat{g}_{\mathrm{R}}(\infty)(1-c \sqrt{z} \exp (-z)+\ldots)
$$

The situation may however be slightly more complicated due to our particular definition of $\xi^{\text {eff }}$. Indeed in the continuum limit at fixed physical volume we expect $G_{2}(0) / G_{2}\left(k_{0}\right) \rightarrow$ 
$G(0) / G(k)$ where $k \sim K_{0}=\left(2 \pi M_{\mathrm{R}} / z, 0\right)$ and the continuum expressions are in finite physical volume. On the other hand for the continuum two point function defined in infinite volume

$$
\frac{1}{K_{0}^{2}}\left[G(0) / G\left(K_{0}\right)-1\right] \sim \frac{1}{M_{\mathrm{R}}^{2}}\left[1-\left(\frac{2 \pi}{z}\right)^{2}\left(\gamma_{2}-1\right)\right] .
$$

In our simulations the values of $2 \pi / z$ are $\sim 1$ i.e. not so small; nevertheless at such values the correction factor on the rhs of (4.12) only deviates from 1 by the order $10^{-3}$. This deviation is much smaller than the statistical accuracy of our simulations, and hence we ignore these additional effects in our analyses of the lattice data.

\section{The Ising model}

The particular field theory we are considering in this section is that obtained from the Ising model in zero external field for $0<T-T_{\mathrm{c}} \rightarrow 0$. The spin-spin correlation functions in the scaling limit are known exactly from the work of $\mathrm{Wu}$ et al [19, and from this knowledge Sato, Miwa and Jimbo [20] found that the S-matrix operator was given by

$$
\mathbf{S}=(-1)^{\mathbf{N}(\mathbf{N}-1) / 2}
$$

where $\mathbf{N}$ is the particle number operator. An energy independent phase is not observable in a scattering experiment; the non-trivial S-matrix (5.1) reflects the fact that the off-shell spin-spin correlation functions are not that of a free field. The continuum limit of the Ising model is also described by a free Majorana field, but this is non-local with respect to the spin field; for a more detailed discussion we refer the reader to the lectures of McCoy 21].

* One can obtain an infinite number of field theories from the Ising model in the presence of an external field $H$ by taking the limit $H \rightarrow 0, T \rightarrow T_{\mathrm{c}}$ with $h=H /\left|T-T_{\mathrm{c}}\right|^{15 / 8}$ fixed. 


\subsection{Form factor determination}

The generalized form factors are given by [22]

$$
\begin{aligned}
& { }^{\text {out }}\left\langle\theta_{1}, \ldots, \theta_{m}|\sigma(0)| \theta_{m+1}, \ldots, \theta_{N}\right\rangle^{\text {in }}= \\
& (2 i)^{(N-1) / 2} \prod_{1 \leq i<j \leq m} T\left(\left|\theta_{i}-\theta_{j}\right|\right) \prod_{1 \leq r \leq m<s \leq N} \frac{\mathcal{P}}{T\left(\theta_{r}-\theta_{s}\right)} \prod_{m<k<l \leq N} T\left(\left|\theta_{k}-\theta_{l}\right|\right),
\end{aligned}
$$

with $N$ an odd (positive) integer. We evaluate the dominant contribution to the coupling using Eq. (3.7). The non-integrated part (3.31) vanishes. For the integral (3.34) we need $f_{1}(\theta)$, which is readily obtained from $(5.2)$,

$$
f_{1}(\theta)=-2 i T(2 \theta) / T^{2}(\theta)
$$

Thus the dominant contribution to $\gamma_{4}$ is

$$
\gamma_{4 ; 121}=\frac{1}{2 \pi} \int_{0}^{\infty} \mathrm{d} u\left[\frac{T^{2}(2 u)}{T^{4}(u) \operatorname{ch}^{2} u}-\frac{16}{u^{2}}\right]=-\frac{5}{2}-\frac{47}{6 \pi}
$$

Numerically this gives $\gamma_{4 ; 121}=-4.993427441(1)$ or $g_{\mathrm{R}} \approx 14.98$ in the leading approximation.

The simplicity of the form factors (5.2) also makes the Ising model a good testing ground for the computation of the sub-leading contributions, to which we turn now. The evaluation of the spectral moments (2.39), (2.43) is straightforward. For $m=3,5,7$ the results are given in Table 1 .

\begin{tabular}{r|r|r}
\hline$m$ & \multicolumn{1}{|c}{$\gamma_{2 ; m}$} & $\delta_{2 ; m}$ \\
\hline \hline 3 & $8.1446256566(1) \times 10^{-4}$ & $1.094(1) \times 10^{-5}$ \\
5 & $7.96(1) \times 10^{-7}$ & $2.22(1) \times 10^{-10}$ \\
7 & $7.8(1) \times 10^{-10}$ & $4.6(1) \times 10^{-15}$ \\
\hline
\end{tabular}

Table 1: $m$-particle contributions to $\gamma_{2}, \delta_{2}$ in the Ising model

Table 1 suggests that the series (2.43) converge extremely rapidly and we would estimate

$$
\gamma_{2}=1+8.15259(1) \times 10^{-4}, \quad \delta_{2}=1+1.094(1) \times 10^{-5}
$$

where the estimated errors come both from the numerical integration and from estimating the contributions of the higher particle terms. To get some check on this we may consider 
the ratio $\delta_{2} / \gamma_{2}$ for which from the leading terms (5.5) we get $\delta_{2} / \gamma_{2}=0.999196336(11)$. This is in excellent agreement with the result $\delta_{2} / \gamma_{2}=0.99919633$ of Campostrini et al. [23], which they obtained by numerical evaluation of the exact formula for the 2-point function if of $\mathrm{Wu}$ et al. [19].

The evaluation of $\gamma_{4}$ is more involved. In order to gain insight into the rate of decay of the higher particle contributions as well as their sign pattern we pushed the computation up to $k+l+m \leq 8$. The $k+l+m=8$ contributions in particular turned out to be a formidable computation despite the deceptive simplicity of the form factors. The computation is based on the formulae (3.9), (3.56) and similar ones for $(m, 2, m)$, with $m$ odd, and for $(1, l, 3)$, with $l$ even. To give the reader a chance to follow the computations we have collected some intermediate results in Appendices B, C. The final results for the contributions of the $k$-l-m intermediate states with $k+l+m \leq 8$ to $\gamma_{4}$ are summarized in Table 2 .

\begin{tabular}{c|c}
\hline$k, l, m$ & $\gamma_{4 ; k l m}$ \\
\hline \hline $1,2,1$ & $-4.993427441(1)$ \\
\hline $1,2,3$ & $0.046310(1)$ \\
$1,4,1$ & $-0.002653(1)$ \\
\hline $3,2,3$ & $0.0002884(3)$ \\
$1,4,3$ & $-0.0000420(5)$ \\
$1,2,5$ & $0.00002562(2)$ \\
$1,6,1$ & $-0.0000040(1)$ \\
\hline
\end{tabular}

Table 2: $k$-l-m-particles contributions to $\gamma_{4}$ in the Ising model

The rapid decay of the terms is manifest. Increasing $k+l+m$ by 2 gives a contribution roughly two orders of magnitude smaller than the previous one. The sign pattern appears to follow the rule: $\operatorname{Sign}\left(\gamma_{4 ; k l m}\right)=\operatorname{Sign}(k+m-l-1)$. Further terms with larger differences $|k-l|,|l-m|$ are suppressed as compared to those with smaller ones. In view of Table 2 we would thus (conservatively) estimate the $k+l+m \geq 10$ particle contributions to be $\leq 10 \%$ of the sum of the $k+l+m=8$ contributions. This gives

$$
\gamma_{4}=-4.90321(3)
$$

\footnotetext{
†this famous Fredholm determinant (solving the Painlevé III equation) is basically the summed up FF series; see e.g. 24.
} 
Inserting into (2.42) with $\gamma_{2}, \delta_{2}$ taken from (5.5) then yields our final result

$$
g_{\mathrm{R}}=14.6975(1)
$$

This amounts to a determination of $g_{\mathrm{R}}$ to within $<0.001 \%$. For comparison we collected the results of some previous determinations in Table 3 below.

\begin{tabular}{c|c}
\hline Method & Value for $g_{\mathrm{R}}$ \\
\hline \hline High temperature & $14.6943(17)$ [13], 14.67(5) [25] \\
Borel summation & $15.5(8)$ 26] \\
Monte Carlo & $14.3(1.0)$ [27 \\
\hline
\end{tabular}

Table 3: Previous determinations of $g_{\mathrm{R}}$ in the Ising model

Finally we would like to mention that an analogous 4-point coupling $h_{\mathrm{R}}$ can be defined at criticality $T=T_{\mathrm{c}}$ by sending the magnetic field $H$ to zero. Of course in this case the definition of Binder's cumulant has to be modified appropriately to take into account the fact that the field has non-vanishing vacuum expectation value. Remarkably $h_{\mathrm{R}}$ can be computed exactly by taking advantage of the fact that the small $H$ behavior of the partition function is known exactly 28 . The final result is $h_{\mathrm{R}}=-\frac{609 \pi}{4}=-478.307$.

\subsection{Recent Monte Carlo simulation of the Ising model}

Our Monte Carlo investigation of $g_{\mathrm{R}}$ was performed on several IBM RISC 6000 workstations at the Werner-Heisenberg-Institut.

In this subsection $\xi^{\text {eff }}$ is denoted simply by $\xi$. We studied the dependence on the lattice spacing by running at $\beta=.418(\xi=10.839936), \beta=.4276(\xi=18.924790)$ and $\beta=$ $.433345(\xi=33.873923)$ on lattices of size $L=80, L=140$ and $L=250$, respectively. These values were chosen in such a way that they have almost exactly the same value of $z=L / \xi \approx 7.4$. Figure 1 shows that there is no significant dependence on the lattice spacing (i.e. $\xi$ ). Therefore we decided to use all the data together to study the finite size effects.

We studied the finite size dependence by measuring in addition $g_{\mathrm{R}}$ on lattices of size $L=40,60,80,140$ at $\beta=.418,(\xi=10.839936)$. Finite size scaling works very well, i.e. 


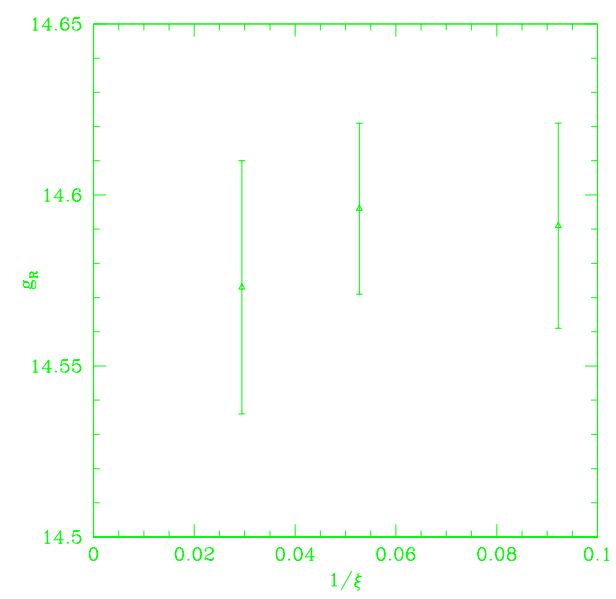

Figure 1: The data for $g_{\mathrm{R}}$ at $z \approx 7.4$ for different lattice spacings

the results only depend on $z=L / \xi$. The dependence on $z$ is still quite well described by Eq. (4.11). This can be seen in figure 2. A least square fit produces

$$
c=3.91(3), \quad \hat{g}_{\mathrm{R}}(\infty)=14.69(2) .
$$

The fit quality is not fantastic $\left(\chi^{2}=2.4\right.$ per d.o.f. $)$ but acceptable. So our final Monte Carlo estimate for $g_{\mathrm{R}}$ is

$$
g_{\mathrm{R}}=14.69(2)
$$

We report our numbers in Table 4 . In this table we also indicate the number of measurements. These were performed using the cluster algorithm as follows: one run consisted of 100,000 clusters used for thermalization, followed by 20,000 sweeps of the lattice used for measurements. Each run was repeated after changing the initial configuration. One such run was considered as one independent measurement. The error was computed out of this sample by using the jack-knife method.

Our estimated value for $g_{\mathrm{R}}$ in Eq. (5.9) is in very good agreement with the values from the analysis of the high temperature expansion given in Table 3; it is also consistent with the value Eq. (5.7) obtained from the form factor construction. 


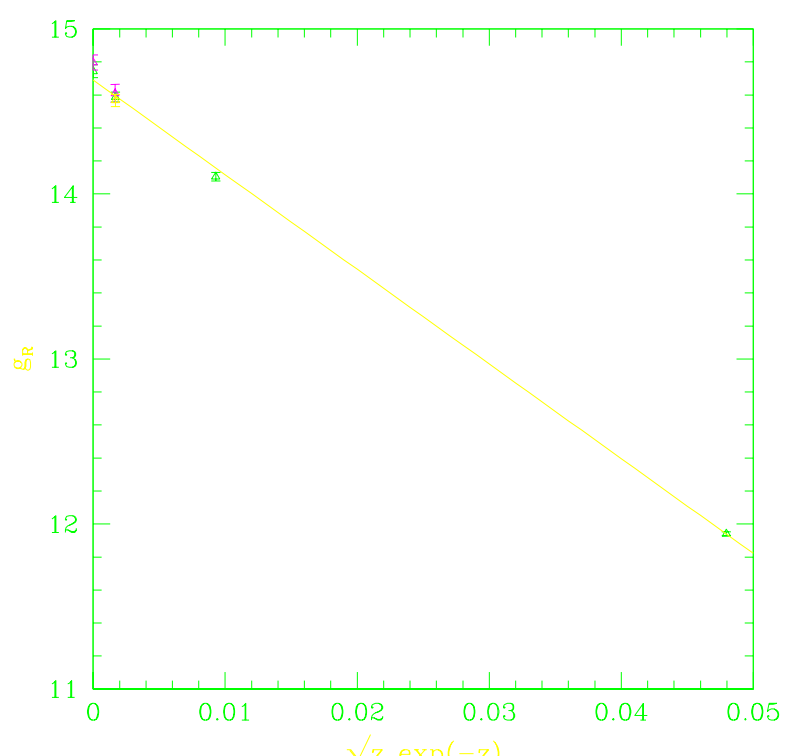

Figure 2: The data for $g_{\mathrm{R}}$ vs $\sqrt{z} \exp (-z)$ together with the fit

\section{The XY-model}

In this section we compute the leading contribution to the four-point coupling in the two-dimensional $\mathrm{O}(2)$ nonlinear $\sigma$-model better known as the XY-model. Starting from the lattice formulation, after a chain of mappings consisting of several steps the model is transformed to a system equivalent to the two-dimensional Coulomb gas. The continuum limit of the Coulomb gas model (corresponding to the Kosterlitz-Thouless critical point [29]) is thought to have a dual description in terms of a Sine-Gordon model at the (extremal) Sine-Gordon coupling $\beta^{2}=8 \pi$. For a review of the XY-model, see [30].

In the following we will start by discussing the XY-model S-matrix. The next step is to solve the Smirnov equations for the three-particle form factors, which enter the formula for the leading term. A general method for finding the Sine-Gordon form factors is given in [31]. This extends the results of Smirnov [6], where the form factors for an even number of particles were found. The spin three-particle form factor we are interested in is probably similar to the three-particle form factor of the fermion operator (corresponding to the equivalent massive Thirring-model description), explicitly given in [31]. Here however we need the three-particle form factor only for special rapidities and we found it simpler to obtain this special version by going back to the functional equations. It is then used to numerically evaluate the leading contribution to $g_{\mathrm{R}}$. 


\begin{tabular}{l|c|c|c|c|c}
\multicolumn{1}{c|}{$\beta$} & $L$ & $\#$ of runs & $\xi^{\text {eff }}$ & $\chi$ & $g_{\mathrm{R}}$ \\
\hline \hline .418 & 40 & 200 & 10.839936 & $163.54(13)$ & $11.941(11)$ \\
\hline .418 & 60 & 200 & 10.839936 & $172.81(11)$ & $14.104(26)$ \\
\hline .418 & 80 & 321 & 10.839936 & $173.94(6)$ & $14.587(30)$ \\
\hline .418 & 140 & 300 & 10.839936 & $174.08(4)$ & $14.743(39)$ \\
\hline .4276 & 140 & 100 & 18.924790 & $455.34(35)$ & $14.610(54)$ \\
\hline .4276 & 250 & 225 & 18.924790 & $455.90(12)$ & $14.796(46)$ \\
\hline .433345 & 250 & 202 & 33.873923 & $1254.48(72)$ & $14.567(37)$ \\
\hline
\end{tabular}

Table 4: Ising data for $\chi=G_{2}(0)$ and $g_{\mathrm{R}}(\beta, L)$

\subsection{The XY-model S-matrix}

We will regard the XY-model as the $n=2$ member of the family of $\mathrm{O}(n) \sigma$-models. Recall that the formulae (3.2), (3.4) have a smooth $n \rightarrow 2$ limit; this has been noted and commented on previously by Woo [32]. In this limit

$$
\sigma_{1}(\theta)=\frac{\theta}{(i \pi-\theta)} s_{2}(\theta), \quad \sigma_{2}(\theta)=0, \quad \sigma_{3}(\theta)=s_{2}(\theta)
$$

and

$$
s_{2}(\theta)=-\exp \left\{2 i \int_{0}^{\infty} \frac{d \omega}{\omega} \sin (\theta \omega) \tilde{K}_{2}(\omega)\right\}, \quad \tilde{K}_{2}(\omega)=\frac{e^{-\frac{\pi \omega}{2}}}{2 \operatorname{ch} \frac{\pi \omega}{2}}
$$

In this paper we will assume that the spectrum of the XY-model in the (massive) continuum limit consists of an $\mathrm{O}(2)$ doublet of massive particles whose S-matrix is given by (6.1) with (6.2). Of course, taking the formal $n \rightarrow 2$ limit of the bootstrap results valid for $n \geq 3$ would not be convincing in itself, but (6.1,6.2) actually coincide with the $\beta^{2} \rightarrow 8 \pi$ limit of the Sine-Gordon S-matrix, the prediction of the Kosterlitz-Thouless theory! The S-matrix (6.1) and the corresponding scattering states as a consequence have a $\mathcal{U}_{q=-1}(\mathrm{su}(2))$ Hopf algebra symmetry, which as a Lie algebra is isomorphic to $\mathrm{su}(2)$. The latter is an explicit symmetry in the alternative chiral Gross-Neveu formulation of the model [30].

\subsection{The three particle form factor}

Next we calculate the three-particle form factor at the special rapidities necessary to compute the leading contribution (3.34). For this purpose we note that the equations for 
the functions $k, l$ given in subsection 3.2 can relatively easily be solved in this particular case $n=2$. We first note that Eq. (3.54) simplifies

$$
\begin{aligned}
K(i \pi-\theta) & =K(i \pi+\theta), \\
K(i \pi+\theta) & =\frac{1}{2 i \pi \theta}\{(i \pi-\theta)(i \pi-2 \theta) L(i \pi-\theta)-(i \pi+\theta)(i \pi+2 \theta) L(i \pi+\theta)\} .
\end{aligned}
$$

Inserting (3.55) yields

$$
K(-i \pi-\theta)=\frac{3 i \pi-2 \theta}{3 i \pi+2 \theta} \cdot \frac{i \pi-2 \theta}{i \pi+2 \theta} K(-i \pi+\theta) .
$$

Luckily a term proportional to $K(i \pi-\theta)$ drops out here and one is left with the simple form (6.4). This can easily be converted into the form (D.1) and solved as

$$
K(i \pi-\theta)=(2 \theta-5 \pi i)(2 \theta-7 \pi i) e^{D\left(\frac{\theta}{2}\right)} \phi\left(i \operatorname{ch} \frac{\theta}{2}\right) .
$$

Here

$$
D(\theta)=\Delta_{\frac{1}{4}}(\theta)+\Delta_{\frac{3}{4}}(\theta)
$$

in the notation of Appendix D and $\phi(z)$ is a polynomial function to be determined later. Since $Y(\theta)$ already has the right singularity structure the functions $K(\theta)$ and $L(\theta)$ are analytic in the physical strip. The residue axioms determine their value at $\theta=0$ and $\theta=\frac{i \pi}{2}$ as

$$
\begin{aligned}
K(0) & =0, & L(0) & =1, \\
K\left(\frac{i \pi}{2}\right) & =\frac{u_{0}}{u^{2}\left(\frac{i \pi}{2}\right)}, & L\left(\frac{i \pi}{2}\right) & =-s_{2}\left(\frac{i \pi}{2}\right) K\left(\frac{i \pi}{2}\right) .
\end{aligned}
$$

So far we have established that the solution can be expressed in terms of

$$
Y(\theta)=-2 i \frac{\operatorname{ch}^{3} \frac{\theta}{2}}{\operatorname{sh} \frac{\theta}{2} \operatorname{ch} \theta} e^{2 \Delta(i \pi+\theta)+\Delta(2 \theta)-\Delta(0)}
$$

and

$$
K(\theta)=(2 \theta+3 \pi i)(2 \theta+5 \pi i) e^{D\left(\frac{i \pi-\theta}{2}\right)} \phi\left(\operatorname{sh} \frac{\theta}{2}\right) .
$$

The polynomial $\phi(z)$ can be determined using the residue constraints (6.7), which we can rewrite as

$$
\begin{aligned}
K(0) & =0, & K^{\prime}(0) & =\frac{2}{i \pi} \\
K\left(\frac{i \pi}{2}\right) & =e^{\Delta(0)-2 \Delta\left(\frac{i \pi}{2}\right)}, & K\left(-\frac{i \pi}{2}\right) & =-e^{\Delta(0)-\Delta\left(\frac{i \pi}{2}\right)-\Delta\left(\frac{-i \pi}{2}\right)} .
\end{aligned}
$$


Using (6.8) and (D.4) one sees that for real $\theta \rightarrow \infty$

$$
|Y(\theta)| \sim e^{-\theta} \theta^{\frac{3}{4}} .
$$

This can be used to infer that the polynomial $\phi(z)$ can be at most second order, otherwise the integral contribution to the leading term would diverge. Taking into account that $K(0)=0$ and the requirement of real analyticity one must have

$$
\phi(z)=i \phi_{1} z+\phi_{2} z^{2}
$$

for real constants $\phi_{1}$ and $\phi_{2}$. Now it is easy to see that (6.7) determines $\phi_{1}$ as

$$
\phi_{1}=\frac{4}{15 \pi^{3}} e^{-D\left(\frac{i \pi}{2}\right)} .
$$

In order to determine $\phi_{2}$ we employ the following identities [33]

$$
\begin{aligned}
& p(\alpha):=\exp \left\{\int_{0}^{\infty} \mathrm{d} \omega \frac{\operatorname{ch}(\alpha \pi \omega)-1}{\operatorname{sh}(\pi \omega)} e^{-\pi \omega}\right\}=\frac{\alpha \pi}{2} \frac{1}{\sin \left(\frac{\alpha \pi}{2}\right)}, \\
& q(\alpha):=\exp \left\{\int_{0}^{\infty} \mathrm{d} \omega \frac{\operatorname{ch}(\alpha \pi \omega)-1}{\operatorname{sh}(\pi \omega)} e^{-2 \pi \omega}\right\}=\frac{1-\alpha^{2}}{\cos \left(\frac{\alpha \pi}{2}\right)},
\end{aligned}
$$

to obtain

$$
\exp \left\{\Delta(0)-2 \Delta\left(\frac{i \pi}{2}\right)+D\left(\frac{i \pi}{2}\right)-D\left(\frac{i \pi}{4}\right)\right\}=p(1) \frac{q^{2}(1)}{q\left(\frac{3}{2}\right)}=\frac{16 \sqrt{2}}{5 \pi}
$$

This can be used to show that (6.10) is satisfied for the choice $\phi_{2}=0$. Thus $\phi\left(\operatorname{sh} \frac{\theta}{2}\right)=$ $i \phi_{1} \operatorname{sh} \frac{\theta}{2}$, and since $4 \theta L(\theta)=(i \pi-2 \theta) K(\theta)-(i \pi+2 \theta) K(-\theta)$, both $k(\theta)=Y(\theta) K(\theta)$ and $l(\theta)=Y(\theta) L(\theta)$ are known explicitly for the XY model.

\subsection{Calculation of the leading contribution}

Having all the ingredients at our disposal we can compute the leading term (3.7) of the intrinsic coupling. Firstly from (3.32) we have for $n=2$

$$
\gamma_{4 ; 121}^{(I)}=\frac{4}{\pi}(\ln 4-1)
$$


Further substituting the explicit results for the functions $k, l$ obtained above into Eq. (3.41) and evaluating the resulting expression numerically we obtain

$$
\gamma_{4 ; 121}^{(I I)}=-5.14902(1)
$$

and hence

$$
\gamma_{4 ; 121}=\gamma_{4 ; 121}^{(I)}+\gamma_{4 ; 121}^{(I I)}=-4.65718
$$

Thus the leading contribution to the XY-model four-point coupling is

$$
g_{\mathrm{R}}=-2 \frac{\gamma_{4}}{\gamma_{2} \delta_{2}} \approx-2 \gamma_{4 ; 121}=9.314
$$

Since $\gamma_{2} \delta_{2}>1$ and since the next leading contributions to $\gamma_{4}$ are probably positive (as they are in the Ising and $\mathrm{O}(3)$ models), we expect that the true value of $g_{\mathrm{R}}$ will be less than that given in 6.20 ) (probably by $2-4 \%$ ).

\subsection{Comparison with lattice results}

For the XY model with standard action Kim [8] gives the value

$$
g_{\mathrm{R}}=8.89(20)
$$

for $\beta=1 / 0.98$. We are in the process of producing higher precision Monte Carlo data for this model; so far we can only give a preliminary result, obtained on a lattice of size $L=500$ at $\beta=1.0174$ :

$$
g_{\mathrm{R}}=9.14(12),
$$

We will return to this issue in a separate publication, where we intend to analyze the finite size corrections as well as the lattice artifacts.

We also wish to mention the results from the high temperature expansion: Butera and Comi [12] obtain

$$
g_{\mathrm{R}}=9.15(10)
$$

whereas Pelissetto and Vicari [13] give

$$
g_{\mathrm{R}}=9.01(5)
$$

So there is an overall rough agreement between the lattice and the form factor results, but the precision is not comparable to that obtained for the Ising model. 


\section{The $\mathrm{O}(3)$ nonlinear sigma-model}

The $\mathrm{O}(3)$ nonlinear sigma model is an important testing ground for quantum field theoretical scenarios in nonabelian gauge theories. The form factor technique has been particularly fruitful in studying its possible off-shell dynamics and can be confronted with what can be achieved by perturbation theory or numerical simulations [34]. The intrinsic coupling has been computed before by a number of different techniques; we compare the results with ours at the end of this section. The present form factor determination takes as usual the Zamolodchikov two-particle S-matrix [3] as its starting point; it is given by Eqs. 3.1, 3.2 with $n=3$ and

$$
s_{2}(\theta)=\frac{\theta-\pi i}{\theta+\pi i}
$$

The corresponding kernel (3.4) is simply given by

$$
\tilde{K}_{3}(\omega)=e^{-\pi \omega}
$$

\subsection{Form factor determination of $g_{\mathrm{R}}$}

Following the by now routine procedure we first collect the ingredients for the evaluation of the dominant $(1,2,1)$ contribution to the intrinsic coupling. From (3.32) one readily finds for $n=3$

$$
\gamma_{4 ; 121}^{(I)}=\frac{4}{\pi}
$$

The $\mathrm{O}(3)$ form factors have been computed in [6, 34, 35]. In particular the reduced 3 -particle form factor $\mathcal{G}$ in Eq. (3.21) is given by

$\mathcal{G}_{a_{1} a_{2} a_{3}}^{a}\left(\theta_{1}, \theta_{2}, \theta_{3}\right)=\tau_{3}\left(\theta_{1}, \theta_{2}, \theta_{3}\right)\left\{\delta_{a_{1}}^{a} \delta_{a_{2} a_{3}}\left(\theta_{3}-\theta_{2}\right)+\delta_{a_{2}}^{a} \delta_{a_{1} a_{3}}\left(\theta_{1}-\theta_{3}-2 \pi i\right)+\delta_{a_{3}}^{a} \delta_{a_{1} a_{2}}\left(\theta_{2}-\theta_{1}\right)\right\}$,

where

$$
\begin{aligned}
\tau_{N}\left(\theta_{1}, \ldots, \theta_{N}\right) & =\prod_{1 \leq i<j \leq N} \tau\left(\theta_{i}-\theta_{j}\right), \\
\tau(\theta) & =\frac{\pi(\theta-i \pi)}{\theta(2 \pi i-\theta)} \tanh \frac{\theta}{2} .
\end{aligned}
$$


Correspondingly the functions $k, l$ parametrizing $f_{b}$ via Eq. (3.40) are for $n=3$ explicitly given by

$$
k(\theta)=\frac{2 \theta}{\pi i-\theta} l(\theta), \quad l(\theta)=\frac{\pi^{3} T^{2}(2 \theta) \theta(2 \theta-\pi i)}{4 T^{4}(\theta)\left(\pi^{2}+\theta^{2}\right)^{2}} .
$$

Plugging this into the general formula Eq. (3.41) yields

$$
\gamma_{4 ; 121}^{(I I)}=\frac{1}{8 \pi} \int_{0}^{\infty} \mathrm{d} u\left\{\frac{\pi^{6} u^{2}\left(4 u^{2}+\pi^{2}\right)\left(2 u^{2}+\pi^{2}\right)}{4\left(u^{2}+\pi^{2}\right)^{5}} \frac{T^{4}(2 u)}{T^{8}(u) \operatorname{ch}^{2} u}-\frac{64}{u^{2}}\right\} .
$$

Numerically we then obtain $\gamma_{4 ; 121}=-4.16835492(1)$, so that as a first approximation $g_{\mathrm{R}} \approx-\frac{5}{3} \gamma_{4 ; 121}=6.9472$. This is already in rough agreement with other determinations in the continuum theory: the $1 / n$, the $\epsilon$ - and the $g$-expansions [13, 7]. The leading order $1 / n$ computations have been performed in [36]. For the spectral integrals the result is

$$
\gamma_{2}=1+0.00671941 \frac{1}{n}+O\left(\frac{1}{n^{2}}\right), \quad \delta_{2}=1+0.00026836 \frac{1}{n}+O\left(\frac{1}{n^{2}}\right)
$$

and for the coupling [10]

$$
g_{\mathrm{R}}=\frac{8 \pi}{n}\left[1-0.602033 \frac{1}{n}+O\left(\frac{1}{n^{2}}\right)\right] .
$$

which gives the approximation $g_{\mathrm{R}} \approx 6.70$ for the case $n=3$. The results from the other methods are given in Table 7. Considering the rather short series in each case it is amazing how well the estimates by the various methods agree.

For a more precise determination we now return to the form factor approach and examine the sub-leading contributions. Using the exact form factors [34] the results for the 3- and 5-particle contributions to $\gamma_{2}$ and $\delta_{2}$ are readily evaluated and are listed in Table 5 .

\begin{tabular}{r|r|r}
\hline$m$ & \multicolumn{1}{|c}{$\gamma_{2 ; m}$} & \multicolumn{1}{c}{$\delta_{2 ; m}$} \\
\hline \hline 3 & $1.67995(1) \times 10^{-3}$ & $3.46494(1) \times 10^{-5}$ \\
5 & $6.622(1) \times 10^{-6}$ & $7.114(1) \times 10^{-9}$ \\
\hline
\end{tabular}

Table 5: $m$-particle contribution to $\gamma_{2}, \delta_{2}$ in the $\mathrm{O}(3)$ model

The size of the higher particle contributions to $\gamma_{2}$ and $\delta_{2}$ can roughly be estimated by an off hand extrapolation of Table 5; essentially they are negligible to the desired accuracy. 
The latter could also be justified by referring to a more refined extrapolation scheme, based on the scaling hypothesis of ref. [34. In upshot we obtain

$$
\gamma_{2}=1.001687(1), \quad \delta_{2}=1.000034657(1)
$$

The computation of the sub-leading terms to $\gamma_{4}$ is much more involved. The starting point is again the formulae (3.10) in subsection 3.1. Due to the complexity of the form factors however the computation is feasible only computer aided. The essential steps are given in appendices B,C. The computation has been performed independently by subsets of the authors using slightly different techniques. The final results for the contributions of the $k-l-m$ intermediate states with $k+l+m \leq 6$ to $\gamma_{4}$ are listed in Table 6 .

\begin{tabular}{c|c}
\hline$k, l, m$ & $\gamma_{4 ; k l m}$ \\
\hline \hline $1,2,1$ & $-4.16835492(1)$ \\
$1,2,3$ & $0.051748(1)$ \\
$1,4,1$ & $-0.004065(1)$ \\
\hline
\end{tabular}

Table 6: $k$-l-m-particles contribution to $\gamma_{4}$ in the $\mathrm{O}(3)$ model

The leading 1-2-1 contribution is a factor $\sim 42$ greater in magnitude than the sum of $k-l$ $m$ contributions with $k+l+m=6$. It is difficult to bound the rest of the contributions, especially since the signs appear to be alternating. The computation of the states with $l+m+n=8$ would be quite an undertaking. But assuming that the pattern in Table 6 continues, as it seems to be the case in the Ising model (see Table 2), then we consider the assumption that the sum of the remaining contributions $k+l+m \geq 8$ is $\leq 10 \%$ of the sum of the $k+l+m=6$ contributions to be reasonable and we then obtain

$$
\gamma_{4}=-4.069(10)
$$

and hence our final result

$$
g_{\mathrm{R}}=6.770(17) .
$$

This amounts to a determination of $g_{\mathrm{R}}$ to within $0.3 \%$. For comparison we give some results of other already published determinations in Table 7 . The first two are continuum methods while the last one is based on the lattice regularization. We describe the two lattice techniques in somewhat more detail in the next subsection, including in particular our own recent Monte Carlo results. 


\begin{tabular}{c|c}
\hline Method & Value for $g_{\mathrm{R}}$ \\
\hline \hline$g$-expansion & $6.66(6)$ [7] \\
-expansion & $6.55(8)$ 13 \\
High temperature & $6.56(4)$ 10, 6.6(1) [11 \\
\hline
\end{tabular}

Table 7: Other determinations of $g_{\mathrm{R}}$ in the $\mathrm{O}(3)$ model

\subsection{Lattice computations of $g_{\mathrm{R}}$}

\section{High temperature expansion:}

The analyses of the high temperature expansion for the spectral moments give $\gamma_{2}=$ 1.0013(2) [36] and $\delta_{2}=1.000029(5)$ [37]. The agreement with the FFB values Eq. (7.10) is acceptable; note that these are smaller than that anticipated from the leading order of the $1 / n$ approximation, Eqs. (7.8).

The various Padé approximations show the coupling falling rapidly as $\beta$ increases in the region of small $\beta$, then a region of rather flat behavior after which these approximations show diverse behavior; some analyses indicate that in fact there is a shallow minimum and that the continuum limit is actually approached from below (see e.g. refs. [23, [13]). In ref. [11] Campostrini et al. quote for the case $n=3$ the result $g_{\mathrm{R}}=6.6(1)$, and in a more recent publication Pelissetto and Vicari cite 6.56(4) [13]. Butera and Comi on the other hand are rather cautious, and did not quote a value for the case $n=3$ in ref. [12]; if pressed they would at present cite $g_{\mathrm{R}}=6.6(2)$ [38.

\section{Numerical simulations:}

Monte Carlo computations of $g_{\mathrm{R}}$ have a long history, see e.g. refs. [7, 8]. In order to attempt to match the apparent precision attained in the FFB approach, we recently performed new high-precision measurements. These were performed on several IBM RISC 6000 workstations at the Werner-Heisenberg-Institut. In addition we made use of the SGI 2000 machine of the University of Arizona, especially for the very time consuming simulations on large lattices.

Based on the fixed point action [18] we have measured $g_{\mathrm{R}}$ at three different values of $\beta$ : $0.70,0.85$ and 1.00 , corresponding to correlation length $\xi \approx 3.2,6.0$ and 12.2 , at the values of $z=L / \xi$ in the range $5.4 \ldots 8.2$. The data and their analysis can be found in [9], the final result is $g_{\mathrm{R}}^{\mathrm{FP}}=6.77(2)$.

Monte Carlo measurements with the standard action were performed using a method 


\begin{tabular}{c|r|c|c|c|c|c}
$\beta$ & $L$ & $\#$ of runs & $\xi$ & $\chi$ & $g_{\mathrm{R}}(\xi, L)$ & $g_{\mathrm{R}}(\xi, \infty)$ \\
\hline \hline 1.5 & 80 & 344 & $11.030(7)$ & $175.95(11)$ & $6.553(16)$ & $6.616(16)$ \\
\hline 1.6 & 140 & 370 & $18.950(14)$ & $447.13(34)$ & $6.612(15)$ & $6.668(15)$ \\
\hline 1.7 & 250 & 367 & $34.500(15)$ & $1267.20(57)$ & $6.665(14)$ & $6.730(14)$ \\
\hline 1.8 & 500 & 382 & $64.790(26)$ & $3838.76(1.50)$ & $6.691(15)$ & $6.733(15)$ \\
\hline 1.9 & 910 & 127 & $122.330(74)$ & $11883.0(6.4)$ & $6.737(21)$ & $6.792(21)$ \\
\hline 1.95 & 1230 & 68 & $167.71(17)$ & $20901.4(19.0)$ & $6.792(40)$ & $6.853(40)$ \\
\hline
\end{tabular}

Table 8: $\mathrm{O}(3)$ data for $\xi, \chi=3 G_{2}(0)$ and $g_{\mathrm{R}}$

similar to the cluster estimator of [39]. We have reported the analysis of such simulations already in our earlier paper [9]. But in the meantime we produced more data and we take the opportunity to report them here.

The present status of the results of our simulations are given in Table 8. In this table we also indicate the number of measurements. These were performed using the cluster algorithm as follows: one run consisted of 100,000 clusters used for thermalization, followed by 20,000 sweeps of the lattice used for measurements. Each run was repeated after changing the initial configuration. One such run was considered as one independent measurement. The error was computed out of this sample by using the jack-knife method.

Our measurements were taken at 6 different correlation lengths ranging from about 11 to about 168 on lattices satisfying $L / \xi \approx 7$. To study the finite volume effects, we took in addition data at $\xi \approx 11$ for lattices of sizes $L$ with $L / \xi \approx 5.5,9$ and 13 . As discussed in [9], the finite size effects are well described by the formula (4.11), even at finite (large) correlation lengths. In the $\mathrm{O}(3)$ model the $n=\infty$ value $c=\sqrt{8 \pi}$ fits very well.

But unlike the Ising model, the lattice artifacts are by no means negligible. To study them, we first use Eq. (4.11) to extrapolate our data to $z=\infty$. In this extrapolation we use the effective correlation length $\xi_{\text {eff }}$ and neglect the fact that this is not exactly equal to the exponential correlation length. In Fig. 3 we plot those extrapolated values of $g_{\mathrm{R}}$ against $1 / \xi$ which we identify with $1 / \xi^{\text {eff }}$.

Unfortunately there is no rigorous result concerning the nature of the approach to the continuum limit. At the time of our last analysis [9] the data point at the largest value of $\xi \sim$ 168 was not available. In that paper we fitted the data in the entire range from $\xi \sim 11$ to $\xi \sim 122$ using a Symanzik type ansatz of the form $g_{\mathrm{R}}(\xi)=g_{\mathrm{R}}(\infty)\left[1+b_{1} \xi^{-2} \log \xi+b_{2} \xi^{-2}\right]$, and thereby obtained the result $g_{\mathrm{R}}=6.77(2)$. When we now repeat the same fit for the new data, which in particular includes the new point at $\xi \sim 168$, the result is only slightly 


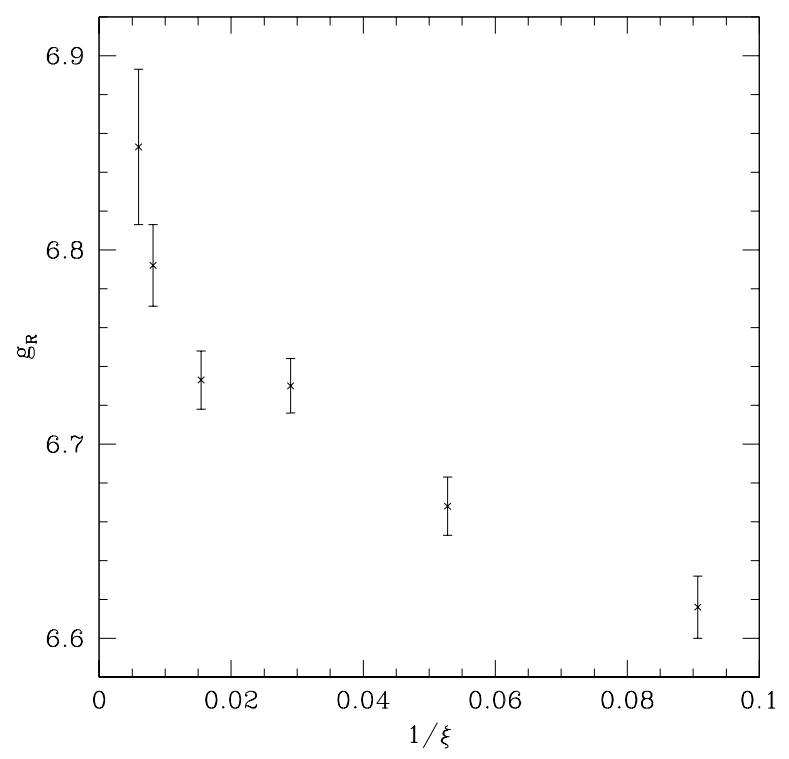

Figure 3: The extrapolated values of $g_{\mathrm{R}}(\xi, \infty)$

changed to $g_{\mathrm{R}}=6.78(2)$ but the quality of the fit becomes poorer. The fact that the two data points closest to the continuum limit lie above 6.78 is in this scenario interpreted as a statistical fluctuation.

On the other hand the present rather large central value at $\xi \sim 168$ could be interpreted as an indication that the continuum limit is approached much slower than conventionally assumed, perhaps as slow as $1 / \ln \xi$ (which may be expected in the $\mathrm{O}(2)$ model [41])! If we adopt this viewpoint it is clear that, although qualitative fits can be made, without further analytic information, our data are not sufficient to make a reliable quantitative extrapolation to the continuum limit. However, independent of the assumed form of the approach to the continuum limit, if the large value at $\xi \sim 168$ is confirmed by more extensive studies it would practically establish a discrepancy between the form factor and the lattice constructions of the $\mathrm{O}(3)$ sigma-model. This point, which needs complete control over all systematic effects, albeit extremely difficult on such large lattices, is certainly worthy of further investigations.

\section{Conclusions}

A new technique to compute the intrinsic 4-point coupling in a large class of two-dimensional 
QFTs has been developed and tested. Starting from the form factor resolution of the 4-point function the termwise zero momentum limit turned out to exist, providing a decomposition of the coupling into terms with a definite number $(k, l, m)$ of intermediate particles. Based on the exactly known form factors these terms can be computed practically exactly and in the models mainly considered (Ising and $\mathrm{O}(3)$ ) were found to be rapidly decaying with increasing particle numbers. There is every reason to expect that this trend continues, which allowed us to equip the results with an intrinsic error estimate. The final results are

$$
\begin{array}{ll}
\text { Ising model: } & g_{\mathrm{R}}=14.6975(1), \\
\mathrm{O}(3) \text { model: } & g_{\mathrm{R}}=6.770(17) .
\end{array}
$$

They amount to a determination of $g_{\mathrm{R}}$ to within $<0.001 \%$ and $0.3 \%$, respectively. In addition we obtained the universal, model-independent formula (A.3) for the dominant contribution to the coupling, which typically seems to account for about $98 \%$ of the full answer. We illustrated its use in testing our proposed bootstrap description of the XYmodel. It would surely also be interesting to apply it e.g. to supersymmetric theories, where alternative techniques are hardly available.

The comparison with the lattice determinations of $g_{\mathrm{R}}$ is quite impressive in the case of the Ising model, where there is also very good agreement between the high temperature and the Monte Carlo determinations. For $\mathrm{O}(2)$ we are so far lacking both precise Monte Carlo and form factor data, but at this preliminary stage there is rough agreement. We intend to return to this model in a separate publication.

The situation in $\mathrm{O}(3)$ is not completely clear: There is a less than perfect agreement between the high temperature result and the new high precision Monte Carlo data, and there is also room for doubt about the agreement between Monte Carlo and form factor. We cannot resolve this question at the moment, mainly because even with our enormous amount of Monte Carlo data it is at the moment not clear what the correct extrapolation to the continuum is.

Acknowledgements: This investigation was supported in part by the Hungarian National Science Fund OTKA (under T030099), and also by the Schweizerische Nationalfonds. The work of M.N. was supported by NSF grant 97-22097. 


\section{A. General formula for the dominant term}

Here we describe the generalization of the formula (3.7) for the dominant 1-2-1 particle contribution to $g_{\mathrm{R}}$ to general integrable QFTs without bound states and operators other than the 'fundamental' field. The latter is particularly natural in the form factor approach because 'fundamental' and 'composite' operators are treated on an equal footing. Thus let $\mathcal{O}_{l}$ be possibly distinct, possibly non-scalar but parity odd operators $\mathcal{O}_{l}$ and write $o_{l}$ for the quantum numbers labeling them. Parallel to (2.13) we define the Green functions by

$$
\tilde{S}_{c}^{o_{1} \ldots o_{L}}\left(k_{1}, \ldots, k_{L}\right)=(2 \pi)^{2} \delta^{(2)}\left(k_{1}+\ldots+k_{L}\right) G^{o_{1} \ldots o_{L}}\left(k_{1}, \ldots, k_{L}\right),
$$

where $\tilde{S}_{c}^{o_{1} \ldots 0_{L}}\left(k_{1}, \ldots, k_{L}\right)$ is the Fourier transform of the connected part of the Euclidean correlation function $\left\langle\mathcal{O}_{1}\left(x_{1}\right) \ldots \mathcal{O}_{L}\left(x_{L}\right)\right\rangle$. The obvious generalization of the intrinsic coupling is

$$
g_{\mathrm{R}}=-\mathcal{N} M^{2} \frac{G^{o_{1} o_{2} o_{3} o_{4}}(0,0,0,0)}{\sum_{j<k} G^{o_{j} o_{k}}(0,0)^{2}} .
$$

Here $M$ is again the mass gap and the constant $\mathcal{N}$ is conveniently adjusted to normalize the 1-particle contribution to the denominator to unity. If $\mathcal{F}_{a}^{o}$ are the constant 1-particle form factors of $\mathcal{O}$, the leading 1-particle contribution to $G^{o_{1} o_{2}}(0,0)$ is just $Z^{o_{1} o_{2}}:=M^{-2} \mathcal{F}_{a}^{o_{1}} C^{a b} \mathcal{F}_{b}^{o_{2}}$, where $C^{a b}$ is the charge conjugation matrix associated with the given S-matrix (c.f. below). Thus we take $\mathcal{N}=\sum_{j<k}\left(Z^{o_{j} o_{k}}\right)^{2}$. With these normalizations the dominant 1-2-1 particle contribution to the coupling (A.2) is

$$
\begin{aligned}
& \left.g_{\mathrm{R}}\right|_{1-2-1}=-\frac{1}{2} \sum_{s \in \mathcal{S}_{4}} D^{o_{s 1} o_{s 2} ; o_{s 3} o_{s 4}}+\int_{0}^{\infty} \frac{\mathrm{d} u}{4 \pi} \sum_{s \in \mathcal{S}_{4}}\left[-\frac{4}{u^{2}} Z^{o_{s 1} o_{s 2}} Z^{o_{s 3} o_{s 4}}\right. \\
& \left.\quad+\frac{1}{16 \mathrm{ch}^{2} u} \mathcal{F}_{a}^{o_{s 1}} \mathcal{F}_{b}^{o_{s 4}} C^{a a_{3}} C^{b b_{3}} \mathcal{F}_{a_{3} a_{2} a_{1}}^{o_{s 2}}(i \pi,-u, u) C^{a_{2} b_{2}} C^{a_{1} b_{1}} \mathcal{F}_{b_{3} b_{2} b_{1}}^{o_{s 3}}(i \pi,-u, u)^{*}\right] .
\end{aligned}
$$

Here the symmetrization is over all elements of the permutation group $\mathcal{S}_{4}$. $D$ is defined in terms of the given bootstrap S-matrix $S_{a b}^{c d}(\theta)$ by

$$
D^{o_{1} o_{2} ; o_{3} o_{4}}=-\left.i \frac{d}{d \theta} S_{c d}^{a b}(\theta)\right|_{\theta=0} \mathcal{F}_{a}^{o_{1}} \mathcal{F}_{b}^{o_{2}} C^{c c^{\prime}} C^{d d^{\prime}} \mathcal{F}_{c^{\prime}}^{o_{3}} \mathcal{F}_{d^{\prime}}^{o_{4}}
$$

and $\mathcal{F}_{a b c}^{o}\left(\theta_{1}, \theta_{2}, \theta_{3}\right)$ is the 3 -particle form factor of $\mathcal{O}$. Taking the results for the $\mathrm{O}(n)$ models as a guideline one would expect that (A.3) typically yields about $98 \%$ of the full answer for the coupling.

In the following we describe the derivation of (A.3). In contrast to that of (3.7) we keep track here of the distributional terms like (2.19) and show explicitly that they cancel out 
in the final answer. In particular this illustrates that the use of the simplifying limit procedure (2.23) is justified.

To fix conventions we first recall the defining relations of a generic bootstrap S-matrix. A matrix-valued meromorphic function $S_{a b}^{d c}(\theta), \theta \in \mathbb{C}$, is called a two particle $S$-matrix if it satisfies the following set of equations. First the Yang-Baxter equation

$$
S_{a b}^{n m}\left(\theta_{12}\right) S_{n c}^{k p}\left(\theta_{13}\right) S_{m p}^{j i}\left(\theta_{23}\right)=S_{b c}^{n m}\left(\theta_{23}\right) S_{a m}^{p i}\left(\theta_{13}\right) S_{p n}^{k j}\left(\theta_{12}\right)
$$

where $\theta_{12}=\theta_{1}-\theta_{2}$ etc. Second unitarity (A.6a,b) and crossing invariance (A.6c)

$$
\begin{aligned}
& S_{a b}^{m n}(\theta) S_{n m}^{c d}(-\theta)=\delta_{a}^{d} \delta_{b}^{c} \\
& S_{a n}^{m c}(\theta) S_{b m}^{n d}(2 \pi i-\theta)=\delta_{a}^{d} \delta_{b}^{c} \\
& S_{a b}^{d c}(\theta)=C_{a a^{\prime}} C^{d d^{\prime}} S_{b d^{\prime}}^{c a^{\prime}}(i \pi-\theta),
\end{aligned}
$$

where (A.6c) together with one of the unitarity conditions (A.6a), (A.6b) implies the other. Further real analyticity and Bose symmetry

$$
\left[S_{a b}^{d c}(\theta)\right]^{*}=S_{a b}^{d c}\left(-\theta^{*}\right), \quad S_{a b}^{d c}(\theta)=S_{b a}^{c d}(\theta)
$$

Finally the normalization condition

$$
S_{a b}^{d c}(0)=-\delta_{a}^{c} \delta_{b}^{d}
$$

The indices $a, b, \ldots$ refer to a basis in a finite dimensional vector space $V$. Indices can be raised and lowered by means of the (constant, symmetric, positive definite) 'charge conjugation matrix' $C_{a b}$ and its inverse $C^{a b}$, satisfying $C_{a d} C^{d b}=\delta_{a}^{b}$. The S-matrix is a meromorphic function of $\theta$. Bound state poles, if any, are situated on the imaginary axis in the so-called physical strip $0 \leq \operatorname{Im} \theta<\pi$. From crossing invariance and the normalization (A.8) one infers that $S_{a b}^{d c}(i \pi)=-C_{a b} C^{d c}$ is always regular, in contrast to $S_{a b}^{d c}(-i \pi)$ which may be singular.

Next we prepare the counterparts of Eqs (2.10), (2.11).

$$
\begin{aligned}
& \left\langle 0\left|\mathcal{O}_{1}\right| \underline{m}\right\rangle\left\langle\underline{m}\left|\mathcal{O}_{2}\right| 0\right\rangle \quad \longleftrightarrow \quad I_{m}^{o_{1} o_{2}}(\theta) \\
& \left\langle 0\left|\mathcal{O}_{1}\right| \underline{k}\right\rangle\left\langle\underline{k}\left|\mathcal{O}_{2}\right| \underline{l}\right\rangle\left\langle\underline{l}\left|\mathcal{O}_{3}\right| \underline{m}\right\rangle\left\langle\underline{m}\left|\mathcal{O}_{4}\right| 0\right\rangle \quad \longleftrightarrow \quad I_{k l m}^{o_{1} o_{2} o_{3} o_{4}}(\omega|\xi| \theta),
\end{aligned}
$$


where

$$
\begin{aligned}
& I_{m}^{o_{1} o_{2}}(\theta)=\mathcal{F}_{A}^{o_{1}}(\theta) C^{A B} \mathcal{F}_{B^{T}}^{o_{2}}\left(\theta^{T}\right) \\
& I_{k l m}^{o_{1} o_{2} o_{3} o_{4}}(\omega|\xi| \theta)=\mathcal{F}_{A}^{o_{1}}(\omega) C^{A B} \mathcal{F}_{B^{T} C}^{o_{2}}\left(\omega^{T} \mid \xi\right) C^{C D} \mathcal{F}_{D^{T} E}^{o_{3}}\left(\xi^{T} \mid \theta\right) C^{E K} \mathcal{F}_{K^{T}}^{o_{4}}\left(\theta^{T}\right) .
\end{aligned}
$$

From the S-matrix exchange relations it follows that $I_{m}^{o_{1} o_{2}}(\theta)$ is a completely symmetric function in $\theta=\left(\theta_{1}, \ldots, \theta_{m}\right)$. Similarly $I_{k l m}^{o_{1} o_{2} o_{3} o_{4}}(\omega|\xi| \theta)$ is symmetric in each of the sets of variables $\omega=\left(\omega_{1}, \ldots, \omega_{k}\right), \xi=\left(\xi_{1}, \ldots, \xi_{l}\right)$ and $\theta=\left(\theta_{1}, \ldots, \theta_{m}\right)$. As before we denote by $V_{m}\left(k_{1}, k_{2}\right)$ and $V_{k l m}\left(k_{1}, k_{2}, k_{3}, k_{4}\right)$ the quantities (2.8) with the integrations over the rapidities performed, where the measure is inherited from (2.25). For simplicity we drop the operator labels $o_{j}$ in the notation. When evaluated at $k_{j}=\left(M \operatorname{sh} \kappa_{j}, 0\right)$ we write $v_{k l m}\left(\kappa_{1}, \kappa_{2}, \kappa_{3}, \kappa_{4}\right)$, etc.

In the next step one inserts the expressions for the generalized form factors in terms of the ordinary form factors; see [16] for an account in the present conventions. For $m=2$ one obtains explicitly

$$
\begin{aligned}
& v_{121}\left(\kappa_{1}, \kappa_{2}, \kappa_{3}, \kappa_{4}\right)=\frac{\mathcal{F}_{a}^{o_{1}} C^{a b} C^{c d} \mathcal{F}_{d}^{o_{4}}}{8 \operatorname{ch}^{2} \kappa_{1} \operatorname{ch}^{2} \kappa_{4}}\left\{\frac{4 \pi \mathcal{F}_{m}^{o_{2}} C^{m n} \mathcal{F}_{n}^{o_{3}} C_{b c}}{\operatorname{ch} \kappa_{2}\left(\operatorname{ch} \kappa_{1}+\operatorname{ch} \kappa_{2}\right)} \delta\left(\kappa_{1}+\kappa_{4}\right)\right. \\
& +\frac{4 \pi \mathcal{F}_{m}^{o_{2}} \mathcal{F}_{n}^{o_{3}}}{\operatorname{ch} \kappa_{2}\left(\operatorname{ch} \kappa_{1}+\operatorname{ch} \kappa_{2}\right)} S_{c b}^{m n}\left(i \pi-\kappa_{1}+\kappa_{2}\right) \delta\left(\kappa_{2}+\kappa_{4}\right) \\
& +\frac{1}{\operatorname{ch} \kappa_{2}\left(\operatorname{ch} \kappa_{1}+\operatorname{ch} \kappa_{2}\right)} \mathcal{F}_{m}^{o_{2}} C^{m n} \mathcal{F}_{c b n}^{o_{3}}\left(\kappa_{4}+i \pi-i \epsilon,-\kappa_{1},-\kappa_{2}\right)^{*} \\
& +\frac{1}{\operatorname{ch} \kappa_{3}\left(\operatorname{ch} \kappa_{3}+\operatorname{ch} \kappa_{4}\right)} \mathcal{F}_{m}^{o_{3}} C^{m n} \mathcal{F}_{b c n}^{o_{2}}\left(-\kappa_{1}+i \pi-i \epsilon, \kappa_{4}, \kappa_{3}\right) \\
& +\int_{0}^{\infty} \frac{\mathrm{d} u}{4 \pi} \frac{1}{4 \operatorname{ch}^{2} \frac{u}{2}+\left(\operatorname{sh} \kappa_{1}+\operatorname{sh} \kappa_{2}\right)^{2}} C^{m k} C^{n l} \\
& \left.\quad \times \mathcal{F}_{b m n}^{o_{2}}\left(-\kappa_{1}+i \pi-i \epsilon, \Lambda_{*}-\frac{u}{2}, \Lambda_{*}+\frac{u}{2}\right) \mathcal{F}_{c k l}^{o 3}\left(\kappa_{4}+i \pi-i \epsilon, \Lambda_{*}-\frac{u}{2}, \Lambda_{*}+\frac{u}{2}\right)^{*}\right\} .
\end{aligned}
$$

The $\kappa_{i} \rightarrow 0$ limit of this expression can be evaluated on general grounds. The key observation is that a three particle form factor has the following universal 'small rapidity' expansion

$$
\begin{aligned}
& \mathcal{F}_{a_{1} a_{2} a_{3}}^{o}\left(\theta_{1}+i \pi-i \epsilon, \theta_{2}, \theta_{3}\right)=\left[\frac{1}{\theta_{12}-i \epsilon}-\frac{1}{\theta_{13}-i \epsilon}\right]\left[2 i\left(C_{a_{1} a_{2}} \mathcal{F}_{a_{3}}^{o}+C_{a_{1} a_{3}} F_{a_{2}}^{o}\right)\right. \\
& \left.+2 C_{a_{1} c}\left(\theta_{13} D_{a_{2} a_{3}}^{c d}-\theta_{12} D_{a_{2} a_{3}}^{d c}\right) \mathcal{F}_{d}^{o}\right], \quad \text { where } D_{a b}^{c d}=-\left.i \frac{d}{d \theta} S_{a b}^{c d}(\theta)\right|_{\theta=0} .
\end{aligned}
$$

This expression is uniquely determined by the following properties: (i) The numerator is linear and boost invariant in the rapidities. (ii) It obeys the (linearized) S-matrix 
exchange relations in $\theta_{2}$ and $\theta_{3}$. (iii) It has simple poles at $\theta_{21}+i \epsilon$ and $\theta_{31}+i \epsilon$ with residues dictated by the form factor 'residue equation' (see e.g. [16] for an account in the present conventions). Using (A.12) in (A.11) one can compute the small $\kappa_{i}$ behavior of $v_{121}$. We denote by $v_{121}^{(I)}$ the contribution from the non-integrated part and by $v_{121}^{(I I)}$ that from the integrated part. One finds

$$
v_{121}^{(I)} \doteq \frac{\pi}{4} Z^{o_{1} o_{3}} Z^{o_{2} o_{4}}\left[\delta\left(\kappa_{1}+\kappa_{3}\right)-\delta\left(\kappa_{1}+\kappa_{4}\right)\right]-\frac{1}{2} D^{o_{1} o_{2} ; o_{3} o_{4}}
$$

where '=' again indicates that both sides give the same result for the symmetrized $\kappa_{i} \rightarrow 0$ limit and $D^{o_{1} o_{2} ; o_{3} o_{4}}=D^{o_{2} o_{1} ; o_{4} o_{3}}=D^{o_{4} o_{3} ; o_{2} o_{1}}$ is given by (A.4).

Analyzing the small $\kappa_{i}$ behavior of the integral in (A.11) (by splitting it according to $\left.\int_{0}^{\infty} \mathrm{d} u=\int_{0}^{\epsilon} \mathrm{d} u+\int_{\epsilon}^{\infty} \mathrm{d} u, \epsilon \rightarrow 0^{+}\right)$one finds a distributional term and a regular one. The result is

$$
\begin{gathered}
v_{121}^{(I I)}=\frac{\pi}{4}\left[Z^{o_{1} o_{4}} Z^{o_{2} o_{3}}+Z^{o_{1} o_{3}} Z^{o_{2} o_{4}}\right] \delta\left(\kappa_{1}+\kappa_{4}\right) \\
+\int_{0}^{\infty} \frac{\mathrm{d} u}{4 \pi}\left[\frac{1}{16 \mathrm{ch}^{2} u} \mathcal{F}_{a}^{o_{1}} \mathcal{F}_{b}^{o_{4}} C^{a a_{3}} C^{b b_{3}} \mathcal{F}_{a_{3} a_{2} a_{1}}^{o_{2}}(i \pi,-u, u) C^{a_{2} b_{2}} C^{a_{1} b_{1}} \mathcal{F}_{b_{3} b_{2} b_{1}}^{o_{3}}(i \pi,-u, u)^{*}\right. \\
\left.-\frac{2}{u^{2}}\left(Z^{o_{1} o_{4}} Z^{o_{2} o_{3}}+Z^{o_{1} o_{3}} Z^{o_{2} o_{4}}\right)\right]
\end{gathered}
$$

where the integrand is regular for $u \rightarrow 0$.

For the generalization of the $\Omega$ term $(2.19)$ one obtains in the 1-particle approximation and in the $\kappa_{i} \rightarrow 0$ limit

$$
\left.\Omega^{o_{1} O_{2} O_{3} O_{4}}\left(k_{1}, k_{2}, k_{3}, k_{4}\right)\right|_{k_{j}=\left(M \operatorname{sh} \kappa_{j}, 0\right)}=-\frac{\pi}{2} \delta\left(\kappa_{1}+\kappa_{2}\right) Z^{o_{1} o_{2}} Z^{o_{3} O_{4}}
$$

Finally, combining (A.13), A.14 and (A.15) according to (2.21) one sees that, - as promised in section 2.2 - all distributional terms drop out when computing the right hand side of (2.21). The final result thus does not depend on any prescription how to take the $\kappa_{i} \rightarrow 0$ limit and is given by (A.3), as asserted. 


\section{B. Computation of the $1-4-1$ contribution}

We start from the general formula Eq. (3.9) with $l=4$. For $\beta_{1} \geq \beta_{2} \geq \beta_{3} \geq \beta_{4}$ we have for one of the factors occurring in (3.10)

$$
\begin{aligned}
\langle a, \alpha| & S^{c}(0)\left|b_{1}, \beta_{1} ; b_{2}, \beta_{2} ; b_{3}, \beta_{3} ; b_{4}, \beta_{4}\right\rangle^{\text {in }}= \\
& \mathcal{F}_{a b_{1} b_{2} b_{3} b_{4}}^{c}\left(i \pi+\alpha-i \epsilon, \beta_{1}, \beta_{2}, \beta_{3}, \beta_{4}\right) \\
& +4 \pi\left\{\delta_{a b_{1}} \delta\left(\alpha-\beta_{1}\right) \mathcal{F}_{b_{2} b_{3} b_{4}}^{c}\left(\beta_{2}, \beta_{3}, \beta_{4}\right)\right. \\
& +\delta\left(\alpha-\beta_{2}\right) \mathcal{F}_{d b_{3} b_{4}}^{c}\left(\beta_{1}, \beta_{3}, \beta_{4}\right) S_{b_{1} b_{2}, d a}\left(\beta_{1}-\beta_{2}\right) \\
& +\delta\left(\alpha-\beta_{3}\right) \mathcal{F}_{d e b_{4}}^{c}\left(\beta_{1}, \beta_{2}, \beta_{4}\right) S_{b_{2} b_{3}, e f}\left(\beta_{2}-\beta_{3}\right) S_{b_{1} f, d a}\left(\beta_{1}-\beta_{3}\right) \\
& +\delta\left(\alpha-\beta_{4}\right) \mathcal{F}_{d e f}^{c}\left(\beta_{1}, \beta_{2}, \beta_{3}\right) S_{b_{3} b_{4}, f g}\left(\beta_{3}-\beta_{4}\right) \\
& \left.\times S_{b_{2} g, e h}\left(\beta_{2}-\beta_{4}\right) S_{b_{1} h, d a}\left(\beta_{1}-\beta_{4}\right)\right\} .
\end{aligned}
$$

For the 5-particle form factor we introduce the reduced form factor through

$$
\mathcal{F}_{a_{1} a_{2} a_{3} a_{4} a_{5}}^{a}\left(\theta_{1}, \theta_{2}, \theta_{3}, \theta_{4}, \theta_{5}\right)=T_{5}\left(\theta_{1}, \ldots, \theta_{5}\right) \mathcal{G}_{a_{1} a_{2} a_{3} a_{4} a_{5}}^{a}\left(\theta_{1}, \theta_{2}, \theta_{3}, \theta_{4}, \theta_{5}\right)
$$

Multiplying out we obtain

$$
I_{141}=\mathcal{K}^{(I)}+\mathcal{K}^{(I I)}+\mathcal{K}^{(I I I)}
$$

where we momentarily omit the arguments $\left(\kappa_{4},-\kappa_{1}, \beta_{1}, \beta_{2}, \beta_{3}, \beta_{4}\right)$. The shorthands are:

$$
\begin{aligned}
\mathcal{K}^{(I)}= & \sum_{b_{1}, b_{2}, b_{3}, b_{4}} \mathcal{F}_{1 b_{1} b_{2} b_{3} b_{4}}^{1}\left(i \pi-\kappa_{1}-i \epsilon, \beta_{1}, \beta_{2}, \beta_{3}, \beta_{4}\right) \\
& \quad \times \mathcal{F}_{1 b_{1} b_{2} b_{3} b_{4}}^{1}\left(i \pi+\kappa_{4}-i \epsilon, \beta_{1}, \beta_{2}, \beta_{3}, \beta_{4}\right)^{*} \\
\mathcal{K}^{(I I)}= & 4 \pi\left\{\delta\left(\beta_{4}+\kappa_{1}\right) \overline{\mathcal{K}}^{(I I)}\left(\kappa_{4},-\kappa_{1}, \beta_{1}, \beta_{2}, \beta_{3}\right)\right. \\
& \left.+\delta\left(\beta_{4}-\kappa_{4}\right) \overline{\mathcal{K}}^{(I I)}\left(-\kappa_{1}, \kappa_{4}, \beta_{1}, \beta_{2}, \beta_{3}\right)^{*}\right\} \\
& +\left(\beta_{4} \leftrightarrow \beta_{3}\right)+\left(\beta_{4} \rightarrow \beta_{2}, \beta_{2} \rightarrow \beta_{3}, \beta_{3} \rightarrow \beta_{4}\right) \\
& +\left(\beta_{4} \rightarrow \beta_{1}, \beta_{1} \rightarrow \beta_{2}, \beta_{2} \rightarrow \beta_{3}, \beta_{3} \rightarrow \beta_{4}\right) \\
\mathcal{K}^{(I I I)}= & (4 \pi)^{2} \overline{\mathcal{K}}^{(I I I)}\left(\kappa_{4},-\kappa_{1}, \beta_{3}, \beta_{4}\right)\left[\delta\left(\beta_{1}+\kappa_{1}\right) \delta\left(\beta_{2}-\kappa_{4}\right)+\left(\beta_{1} \leftrightarrow \beta_{2}\right)\right] \\
& +\left(\beta_{2} \leftrightarrow \beta_{3}\right)+\left(\beta_{2} \rightarrow \beta_{4}, \beta_{4} \rightarrow \beta_{3}, \beta_{3} \rightarrow \beta_{2}\right) \\
& +\left(\beta_{1} \rightarrow \beta_{2}, \beta_{2} \rightarrow \beta_{3}, \beta_{3} \rightarrow \beta_{1}\right)+\left(\beta_{1} \rightarrow \beta_{2}, \beta_{2} \rightarrow \beta_{4}, \beta_{4} \rightarrow \beta_{3}, \beta_{3} \rightarrow \beta_{1}\right) \\
& +\left(\beta_{1} \rightarrow \beta_{3}, \beta_{3} \rightarrow \beta_{2}, \beta_{2} \rightarrow \beta_{4}, \beta_{4} \rightarrow \beta_{1}\right)
\end{aligned}
$$


where

$$
\begin{aligned}
& \overline{\mathcal{K}}^{(I I)}\left(\alpha, \gamma, \beta_{1}, \beta_{2}, \beta_{3}\right) \underset{b_{1}, b_{2}, b_{3}}{\Rightarrow} \mathcal{F}_{b_{1} b_{2} b_{3}}^{1}\left(\beta_{1}, \beta_{2}, \beta_{3}\right) \mathcal{F}_{11 b_{1} b_{2} b_{3}}^{1}\left(i \pi+\alpha-i \epsilon, \gamma, \beta_{1}, \beta_{2}, \beta_{3}\right)^{*} \\
& \overline{\mathcal{K}}^{(I I I)}\left(\kappa_{4},-\kappa_{1}, \beta_{3}, \beta_{4}\right)=\sum_{b_{1} b_{2}, b_{3}, b_{4}} \mathcal{F}_{b_{1} b_{3} b_{4}}^{1}\left(\kappa_{4}, \beta_{3}, \beta_{4}\right) \mathcal{F}_{b_{2} b_{3} b_{4}}^{1}\left(-\kappa_{1}, \beta_{3}, \beta_{4}\right)^{*} S_{1 b_{2}, b_{1} 1}\left(\kappa_{1}+\kappa_{4}\right)(
\end{aligned}
$$

Because of the symmetry in the $\beta_{i}$ arguments of $\mathcal{K}^{(I)}, \overline{\mathcal{K}}^{(I I)}, \overline{\mathcal{K}}^{(I I I)}$ one has

$$
\begin{aligned}
& v_{141}^{(I)}\left(\kappa_{1}, \kappa_{2}, \kappa_{3}, \kappa_{4}\right)= \frac{1}{12288 \pi^{3}} \int_{-\infty}^{\infty} \mathrm{d} \beta_{1} \int_{-\infty}^{\infty} \mathrm{d} \beta_{2} \int_{-\infty}^{\infty} \mathrm{d} \beta_{3} \int_{-\infty}^{\infty} \mathrm{d} \beta_{4} \\
& \times \frac{\delta\left(\beta_{1}, \beta_{2}, \beta_{3}, \beta_{4}, \kappa_{1}, \kappa_{2}\right)}{\sum_{k=1}^{4} \operatorname{ch} \beta_{k}} \mathcal{K}^{(I)}\left(\kappa_{4},-\kappa_{1}, \beta_{1}, \beta_{2}, \beta_{3}, \beta_{4}\right), \\
& v_{141}^{(I I)}\left(\kappa_{1}, \kappa_{2}, \kappa_{3}, \kappa_{4}\right)= \frac{1}{768 \pi^{2}} \int_{-\infty}^{\infty} \mathrm{d} \beta_{1} \int_{-\infty}^{\infty} \mathrm{d} \beta_{2} \int_{-\infty}^{\infty} \mathrm{d} \beta_{3} \frac{1}{1+\sum_{k=1}^{3} \operatorname{ch} \beta_{k}} \\
&\left\{\delta\left(\beta_{1}, \beta_{2}, \beta_{3}, \kappa_{2}\right) \overline{\mathcal{K}}^{(I I)}\left(\kappa_{4},-\kappa_{1}, \beta_{1}, \beta_{2}, \beta_{3}\right)+\delta\left(\beta_{1}, \beta_{2}, \beta_{3},-\kappa_{3}\right) \overline{\mathcal{K}}^{(I I)}\left(-\kappa_{1}, \kappa_{4}, \beta_{1}, \beta_{2}, \beta_{3}\right)^{*}\right\}, \\
& v_{141}^{(I I I)}\left(\kappa_{1}, \kappa_{2}, \kappa_{3}, \kappa_{4}\right)= \frac{1}{64 \pi} \int_{-\infty}^{\infty} \mathrm{d} \beta_{1} \int_{-\infty}^{\infty} \mathrm{d} \beta_{2} \frac{\delta\left(\beta_{1}, \beta_{2}, \kappa_{2}, \kappa_{4}\right)}{2+\sum_{k=1}^{2} \operatorname{ch} \beta_{k}} \overline{\mathcal{K}}^{(I I I)}\left(\kappa_{4},-\kappa_{1}, \beta_{1}, \beta_{2}\right) .
\end{aligned}
$$

The contribution $(I I I)$ is very simple; we can set the $\kappa_{i}$ to zero to obtain

$$
v_{141}^{(I I I)}\left(\kappa_{1}, \kappa_{2}, \kappa_{3}, \kappa_{4}\right)=\frac{1}{128 \pi} \int_{-\infty}^{\infty} \mathrm{d} \beta \frac{1}{\operatorname{ch} \beta(1+\operatorname{ch} \beta)} T^{2}(2 \beta) T^{4}(\beta) k^{(I I I)}(\beta),
$$

where we decomposed

$$
\overline{\mathcal{K}}^{(I I I)}(0,0, \beta,-\beta)=T^{2}(2 \beta) T^{4}(\beta) k^{(I I I)}(\beta)
$$

Writing similarly

$$
\begin{aligned}
& \overline{\mathcal{K}}^{(I I)}\left(\alpha, \gamma, \beta_{1}, \beta_{2}, \beta_{3}\right)=k^{(I I)}\left(\alpha, \gamma, \beta_{1}, \beta_{2}, \beta_{3}\right) \\
& \quad \times \frac{1}{T(\alpha-\gamma)} \prod_{1 \leq i<j \leq 3} T^{2}\left(\beta_{i}-\beta_{j}\right) \prod_{k=1}^{3} \frac{T\left(\beta_{k}-\gamma\right)}{T\left(\beta_{k}-\alpha-i \epsilon\right)},
\end{aligned}
$$

one has

$$
v_{141}^{(I I)}=\sum_{j=1}^{2} v_{141}^{(I I, j)}
$$


with

$$
\begin{aligned}
& v_{141}^{(I I, 1)}\left(\kappa_{1}, \kappa_{2}, \kappa_{3}, \kappa_{4}\right)=\frac{1}{768 \pi^{2}} \frac{1}{T\left(\kappa_{1}+\kappa_{4}\right)} \int_{-\infty}^{\infty} \mathrm{d} \beta_{1} \int_{-\infty}^{\infty} \mathrm{d} \beta_{2} \int_{-\infty}^{\infty} \mathrm{d} \beta_{3} \\
& \quad \times \frac{1}{1+\sum_{k=1}^{3} \operatorname{ch} \beta_{k}} \prod_{1 \leq i<j \leq 3} T^{2}\left(\beta_{i}-\beta_{j}\right) \\
& \quad \times\left\{\delta\left(\beta_{1}, \beta_{2}, \beta_{3}, \kappa_{2}\right) k^{(I I)}\left(\kappa_{4},-\kappa_{1}, \beta_{1}, \beta_{2}, \beta_{3}\right) \prod_{k=1}^{3} T\left(\beta_{k}+\kappa_{1}\right) \frac{\mathcal{P}}{T\left(\beta_{k}-\kappa_{4}\right)}\right. \\
& \left.-\delta\left(\beta_{1}, \beta_{2}, \beta_{3},-\kappa_{3}\right) k^{(I I)}\left(-\kappa_{1}, \kappa_{4}, \beta_{1}, \beta_{2}, \beta_{3}\right)^{*} \prod_{k=1}^{3} T\left(\beta_{k}-\kappa_{4}\right) \frac{\mathcal{P}}{T\left(\beta_{k}+\kappa_{1}\right)}\right\}, \\
& v_{141}^{(I I, 2)}\left(\kappa_{1}, \kappa_{2}, \kappa_{3}, \kappa_{4}\right)=\frac{i}{128 \pi} \int_{-\infty}^{\infty} \mathrm{d} \beta_{1} \int_{-\infty}^{\infty} \mathrm{d} \beta_{2} \frac{\delta\left(\beta_{1}, \beta_{2}, \kappa_{2}, \kappa_{4}\right)}{2+\sum_{k=1}^{2} \mathrm{ch} \beta_{k}} \\
& \quad \times T^{2}\left(\beta_{1}-\beta_{2}\right) \prod_{k=1}^{2} T\left(\beta_{k}-\kappa_{4}\right) T\left(\beta_{k}+\kappa_{1}\right) \\
& \quad \times\left\{k^{(I I)}\left(\kappa_{4},-\kappa_{1}, \beta_{1}, \beta_{2}, \kappa_{4}\right)-k^{(I I)}\left(-\kappa_{1}, \kappa_{4}, \beta_{1}, \beta_{2},-\kappa_{1}\right)^{*}\right\} .
\end{aligned}
$$

In the latter term we can set the $\kappa_{i}$ to zero to obtain

$$
v_{141}^{(I I, 2)}(0,0,0,0)=\frac{-1}{128 \pi} \int_{-\infty}^{\infty} \mathrm{d} \beta \frac{1}{\operatorname{ch} \beta(1+\operatorname{ch} \beta)} T^{2}(2 \beta) T^{4}(\beta) \operatorname{Im}\left[k^{(I I)}(0,0, \beta,-\beta, 0)\right] .
$$

Lastly we turn to the $(I)$ contribution. There are many ways to manipulate the integral into a form more amenable to numerical evaluation. Here we proceed as follows: Writing

$$
\begin{aligned}
\mathcal{K}^{(I)}= & k^{(I)}\left(\kappa_{4},-\kappa_{1}, \beta_{1}, \beta_{2}, \beta_{3}, \beta_{4}\right) \prod_{1 \leq i<j \leq 4} T^{2}\left(\beta_{i}-\beta_{j}\right) \\
& \times \prod_{k=1}^{4} \frac{1}{T\left(\beta_{k}+\kappa_{1}+i \epsilon\right) T\left(\beta_{k}-\kappa_{4}-i \epsilon\right)}
\end{aligned}
$$

we replace the $1 /(x \pm i \epsilon)$ distributions by a sum of products of principal parts and delta functions, thereby obtaining

$$
v_{141}^{(I)}=\sum_{j=1}^{3} v_{141}^{(I, j)} .
$$

The three terms are:

$$
\begin{aligned}
& v_{141}^{(I, 1)}\left(\kappa_{1}, \kappa_{2}, \kappa_{3}, \kappa_{4}\right)=\frac{1}{12288 \pi^{3}} \\
& \quad \int_{-\infty}^{\infty} \mathrm{d} \beta_{1} \int_{-\infty}^{\infty} \mathrm{d} \beta_{2} \int_{-\infty}^{\infty} \mathrm{d} \beta_{3} \int_{-\infty}^{\infty} \mathrm{d} \beta_{4} \frac{\delta\left(\beta_{1}, \beta_{2}, \beta_{3}, \beta_{4}, \kappa_{1}, \kappa_{2}\right)}{\sum_{k=1}^{4} \operatorname{ch} \beta_{k}}
\end{aligned}
$$




$$
\begin{aligned}
& k^{(I)}\left(\kappa_{4},-\kappa_{1}, \beta_{1}, \beta_{2}, \beta_{3}, \beta_{4}\right) \prod_{1 \leq i<j \leq 4} T^{2}\left(\beta_{i}-\beta_{j}\right) \prod_{k=1}^{4} \frac{\mathcal{P}}{T\left(\beta_{k}+\kappa_{1}\right)} \frac{\mathcal{P}}{T\left(\beta_{k}-\kappa_{4}\right)} \\
& v_{141}^{(I, 2)}\left(\kappa_{1}, \kappa_{2}, \kappa_{3}, \kappa_{4}\right)=\frac{i}{1536 \pi^{2}} \frac{1}{T\left(\kappa_{1}+\kappa_{4}\right)} \\
& \int_{-\infty}^{\infty} \mathrm{d} \beta_{1} \int_{-\infty}^{\infty} \mathrm{d} \beta_{2} \int_{-\infty}^{\infty} \mathrm{d} \beta_{3} \frac{1}{1+\sum_{k=1}^{3} \operatorname{ch} \beta_{k}} \prod_{1 \leq i<j \leq 3} T^{2}\left(\beta_{i}-\beta_{j}\right) \\
& \quad \times\left\{\delta\left(\beta_{1}, \beta_{2}, \beta_{3}, \kappa_{2}\right) k^{(I)}\left(\kappa_{4},-\kappa_{1}, \beta_{1}, \beta_{2}, \beta_{3},-\kappa_{1}\right) \prod_{k=1}^{3} T\left(\beta_{k}+\kappa_{1}\right) \frac{\mathcal{P}}{T\left(\beta_{k}-\kappa_{4}\right)}\right. \\
& \left.+\delta\left(\beta_{1}, \beta_{2}, \beta_{3},-\kappa_{3}\right) k^{(I)}\left(\kappa_{4},-\kappa_{1}, \beta_{1}, \beta_{2}, \beta_{3}, \kappa_{4}\right) \prod_{k=1}^{3} T\left(\beta_{k}-\kappa_{4}\right) \frac{\mathcal{P}}{T\left(\beta_{k}+\kappa_{1}\right)}\right\} \\
& v_{141}^{(I, 3)}\left(\kappa_{1}, \kappa_{2}, \kappa_{3}, \kappa_{4}\right)=\frac{-1}{256 \pi} \int_{-\infty}^{\infty} \mathrm{d} \beta_{1} \int_{-\infty}^{\infty} \mathrm{d} \beta_{2} \frac{\delta\left(\beta_{1}, \beta_{2}, \kappa_{2}, \kappa_{4}\right)}{2+\sum_{k=1}^{2} \operatorname{ch} \beta_{k}} \\
& k^{(I)}\left(\kappa_{4},-\kappa_{1}, \beta_{1}, \beta_{2},-\kappa_{1}, \kappa_{4}\right) T^{2}\left(\beta_{1}-\beta_{2}\right) \prod_{k=1}^{2} T\left(\beta_{k}-\kappa_{4}\right) T\left(\beta_{k}+\kappa_{1}\right) .
\end{aligned}
$$

In the latter expression we can set the $\kappa_{i}$ to zero to obtain

$$
v_{141}^{(I, 3)}(0,0,0,0)=\frac{-1}{512 \pi} \int_{-\infty}^{\infty} \mathrm{d} \beta \frac{1}{\operatorname{ch} \beta(1+\operatorname{ch} \beta)} T^{2}(2 \beta) T^{4}(\beta) k^{(I)}(0,0, \beta,-\beta, 0,0) .
$$

For $W^{(1)}:=v_{141}^{(I, 1)}$ we now invoke the identity

$$
\frac{\prod_{1 \leq i<j \leq 4} \operatorname{sh}\left(y_{i}-y_{j}\right)}{\prod_{k=1}^{4} \operatorname{sh}\left(y_{k}+x\right)}=\frac{1}{\operatorname{ch}(x)} \sum_{k=1}^{4} \frac{(-1)^{k} \operatorname{ch}\left(y_{k}\right)}{\operatorname{sh}\left(y_{k}+x\right)} \prod_{1 \leq i<j \leq 4, i \neq k \neq j} \operatorname{sh}\left(y_{i}-y_{j}\right)
$$

to get

$$
W^{(1)}=\lim _{\alpha_{c} \rightarrow \infty}\left[W^{(1)}[A]\left(\alpha_{c}\right)+W^{(1)}[B]\left(\alpha_{c}\right)\right]
$$

with the notation

$$
W^{(1)}[X]\left(\alpha_{c}\right)=\frac{1}{192 \pi^{3}} \int_{-\alpha_{c}}^{\alpha_{c}} \mathrm{~d} \alpha_{1} G_{X}\left(\alpha_{1}\right), \quad X=A, B
$$

Here

$$
G_{A}\left(\alpha_{1}\right)=\frac{\mathcal{P}}{T\left(\alpha_{1}\right)} \int_{-\infty}^{\infty} \mathrm{d} \alpha_{2} \frac{\mathcal{P}}{T\left(\alpha_{2}\right)} \int_{-\infty}^{\infty} \mathrm{d} \alpha_{3} \mathcal{F}_{A}\left(\alpha_{1}, \alpha_{2}, \alpha_{3}\right)
$$




$$
\begin{aligned}
= & \frac{\mathcal{P}}{T\left(\alpha_{1}\right)} \int_{0}^{\infty} \mathrm{d} \alpha_{2} \frac{\mathcal{P}}{T\left(\alpha_{2}\right)} \int_{0}^{\infty} \mathrm{d} \alpha_{3}\left\{\mathcal{F}_{A}\left(\alpha_{1}, \alpha_{2}, \alpha_{3}\right)\right. \\
& \left.-\mathcal{F}_{A}\left(\alpha_{1},-\alpha_{2}, \alpha_{3}\right)-\mathcal{F}_{A}\left(-\alpha_{1}, \alpha_{2}, \alpha_{3}\right)+\mathcal{F}_{A}\left(-\alpha_{1},-\alpha_{2}, \alpha_{3}\right) \cdot\right\}, \\
G_{B}\left(\alpha_{1}\right)= & \frac{G\left(\alpha_{1}\right)}{\operatorname{sh}^{2} \frac{\alpha_{1}}{2}}-\frac{4 G(0)}{\alpha_{1}^{2}},
\end{aligned}
$$

where

$$
G\left(\alpha_{1}\right)=\operatorname{ch}^{2} \frac{\alpha_{1}}{2} \int_{-\infty}^{\infty} \mathrm{d} \alpha_{2} \int_{-\infty}^{\infty} \mathrm{d} \alpha_{3} \mathcal{F}_{B}\left(\alpha_{1}, \alpha_{2}, \alpha_{3}\right) .
$$

In these formulae

$$
\mathcal{F}_{X}\left(\alpha_{1}, \alpha_{2}, \alpha_{3}\right)=\frac{1}{16}\left[\frac{k^{(I)}\left(0,0, \alpha_{1}, \alpha_{2}, \alpha_{3}, \alpha_{4}\right) f_{X}\left(\alpha_{1}, \alpha_{2}, \alpha_{3}, \alpha_{4}\right) \prod_{k=1}^{4} \operatorname{ch}^{2} \frac{\alpha_{k}}{2}}{\operatorname{ch} \alpha_{4}\left(\sum_{m=1}^{4} \operatorname{ch} \alpha_{m}\right) \prod_{i<j} \operatorname{ch}^{2} \frac{\alpha_{i}-\alpha_{j}}{2}}\right]_{\alpha_{4}=\gamma}
$$

where $\gamma$ is given through $\operatorname{sh} \gamma=-\sum_{k=1}^{3} \operatorname{sh} \alpha_{k}$ and

$$
\begin{aligned}
f_{A}\left(\alpha_{1}, \alpha_{2}, \alpha_{3}, \alpha_{4}\right)=- & 3 \operatorname{sh} \frac{\alpha_{1}-\alpha_{3}}{2} \operatorname{sh} \frac{\alpha_{1}-\alpha_{4}}{2} \\
& \times \operatorname{sh} \frac{\alpha_{2}-\alpha_{3}}{2} \operatorname{sh}^{\frac{\alpha_{2}-\alpha_{4}}{2}} \operatorname{sh}^{2} \frac{\alpha_{3}-\alpha_{4}}{2}, \\
f_{B}\left(\alpha_{1}, \alpha_{2}, \alpha_{3}, \alpha_{4}\right)= & \operatorname{sh}^{2} \frac{\alpha_{2}-\alpha_{3}}{2} \operatorname{sh}^{2} \frac{\alpha_{2}-\alpha_{4}}{2} \operatorname{sh}^{2} \frac{\alpha_{3}-\alpha_{4}}{2} .
\end{aligned}
$$

For the $[B]$ contribution it is numerically convenient to decompose

$$
W^{(1)}[B]\left(\alpha_{c}\right) \sim h G(0)+W^{(1)}[B 0]+W^{(1)}[B 1]\left(\alpha_{c}\right),
$$

where

$$
\begin{aligned}
W^{(1)}[B 0] & =\frac{1}{96 \pi^{3}} \int_{0}^{1} \mathrm{~d} \alpha_{1}\left(\frac{G\left(\alpha_{1}\right)-G(0)}{\operatorname{sh}^{2} \frac{\alpha_{1}}{2}}\right), \\
W^{(1)}[B 1]\left(\alpha_{c}\right) & =\frac{1}{96 \pi^{3}} \int_{1}^{\alpha_{c u t}} \mathrm{~d} \alpha_{1} \frac{G\left(\alpha_{1}\right)}{\operatorname{sh}^{2} \frac{\alpha_{1}}{2}},
\end{aligned}
$$

and

$$
h=-\frac{1}{96 \pi^{3}}\left\{4-\int_{0}^{1} \mathrm{~d} \alpha_{1}\left[\frac{1}{\operatorname{sh}^{2} \frac{\alpha_{1}}{2}}-\frac{4}{\alpha_{1}^{2}}\right]\right\}=-0.0014539754 .
$$

Finally we recombine

$$
v_{141}\left(\kappa_{1}, \kappa_{2}, \kappa_{3}, \kappa_{4}\right)=\sum_{j=1}^{3} W^{(j)}\left(\kappa_{1}, \kappa_{2}, \kappa_{3}, \kappa_{4}\right),
$$


with

$$
\begin{aligned}
& W^{(1)}=v_{141}^{(I, 1)} \\
& W^{(2)}=v_{141}^{(I, 2)}+v_{141}^{(I I, 1)} \\
& W^{(3)}=v_{141}^{(I, 3)}+v_{141}^{(I I, 2)}+v_{141}^{(I I I)} .
\end{aligned}
$$

\section{Case $n=1$ :}

Here we simply have (recall the 2-particle S-matrix $=-1$ )

$$
k^{(I)}=16, \quad k^{(I I)}=-8 i, \quad k^{(I I I)}=-4,
$$

from which one sees

$$
\begin{aligned}
& v_{141}^{(I, 2)}(0,0,0,0)=-v_{141}^{(I I, 1)}(0,0,0,0) \\
& v_{141}^{(I, 3)}(0,0,0,0)=v_{141}^{(I I I)}(0,0,0,0)=-\frac{1}{2} v_{141}^{(I I, 2)}(0,0,0,0) .
\end{aligned}
$$

Thus

$$
W^{(2)}=0=W^{(3)},
$$

so that for the Ising case we simply get $v_{141}=W^{(1)}$, with $W^{(1)}$ given by Eq. (B.21) and $k^{(I)}$ by Eq. (B.39). This is, as expected, the same expression as that obtained directly with the form factor written as a product over principal parts as in Eq. (5.2).

\section{Case $n=3$ :}

Firstly for $W^{(1)}$ we obtain

$$
W^{(1)}=-0.0005420(1)
$$

Next for $W^{(2)}$ one has

$$
\begin{aligned}
& W^{(2)}\left(\kappa_{1}, \kappa_{2}, \kappa_{3}, \kappa_{4}\right)=\frac{1}{768 \pi^{2}} \frac{1}{T\left(\kappa_{1}+\kappa_{4}\right)} \int_{-\infty}^{\infty} \mathrm{d} \beta_{1} \int_{-\infty}^{\infty} \mathrm{d} \beta_{2} \int_{-\infty}^{\infty} \mathrm{d} \beta_{3} J\left(\beta_{1}, \beta_{2}, \beta_{3}\right) \\
& \quad \times\left\{\delta\left(\beta_{1}, \beta_{2}, \beta_{3}, \kappa_{2}\right) w^{(2)}\left(\kappa_{1}, \kappa_{4}, \beta_{1}, \beta_{2}, \beta_{3}\right) \prod_{k=1}^{3} T\left(\beta_{k}+\kappa_{1}\right) \frac{\mathcal{P}}{T\left(\beta_{k}-\kappa_{4}\right)}\right. \\
& \left.\quad+\delta\left(\beta_{1}, \beta_{2}, \beta_{3}, \kappa_{3}\right) \tilde{w}^{(2)}\left(\kappa_{1}, \kappa_{4}, \beta_{1}, \beta_{2}, \beta_{3}\right) \prod_{k=1}^{3} T\left(\beta_{k}+\kappa_{4}\right) \frac{\mathcal{P}}{T\left(\beta_{k}-\kappa_{1}\right)}\right\}
\end{aligned}
$$

where

$$
J\left(\beta_{1}, \beta_{2}, \beta_{3}\right)=\frac{1}{1+\sum_{k=1}^{3} \operatorname{ch} \beta_{k}} \prod_{1 \leq i<j \leq 3} T^{2}\left(\beta_{i}-\beta_{j}\right),
$$


and

$$
\begin{aligned}
& w^{(2)}\left(\kappa_{1}, \kappa_{4}, \beta_{1}, \beta_{2}, \beta_{3}\right)= \\
& \quad k^{(I I)}\left(\kappa_{4},-\kappa_{1}, \beta_{1}, \beta_{2}, \beta_{3}\right)+\frac{i}{2} k^{(I)}\left(\kappa_{4},-\kappa_{1}, \beta_{1}, \beta_{2}, \beta_{3},-\kappa_{1}\right), \\
& \tilde{w}^{(2)}\left(\kappa_{1}, \kappa_{4}, \beta_{1}, \beta_{2}, \beta_{3}\right)= \\
& \quad-k^{(I I)}\left(-\kappa_{1}, \kappa_{4},-\beta_{1},-\beta_{2},-\beta_{3}\right)^{*}+\frac{i}{2} k^{(I)}\left(\kappa_{4},-\kappa_{1},-\beta_{1},-\beta_{2},-\beta_{3}, \kappa_{4}\right) .
\end{aligned}
$$

Explicit calculation reveals the fact

$$
\tilde{w}^{(2)}\left(\kappa_{1}, \kappa_{4}, \beta_{1}, \beta_{2}, \beta_{3}\right)=w^{(2)}\left(\kappa_{4}, \kappa_{1}, \beta_{1}, \beta_{2}, \beta_{3}\right)
$$

Now we expand $w^{(2)}$ for small $\kappa_{i}$ :

$$
\begin{aligned}
& w^{(2)}\left(\kappa_{1}, \kappa_{4}, \beta_{1}, \beta_{2}, \beta_{3}\right)=w_{0}\left(\beta_{1}, \beta_{2}, \beta_{3}\right) \\
& \quad+\left(\kappa_{1}+\kappa_{4}\right) w_{1}\left(\beta_{1}, \beta_{2}, \beta_{3}\right)+\left(\kappa_{1}-\kappa_{4}\right) w_{2}\left(\beta_{1}, \beta_{2}, \beta_{3}\right)+O\left(\kappa_{i}^{2}\right) .
\end{aligned}
$$

In fact we do not require $w_{2}$. Note that the functions $w_{i}$ are real, so that in particular

$$
\operatorname{Im} w^{(2)}\left(0,0, \beta_{1}, \beta_{2}, \beta_{3}\right)=0
$$

which is needed to avoid a singularity in $W^{(2)}$ for $\kappa_{i} \rightarrow 0$. Hence

$$
W^{(2)}=W^{(2)}[A]+W^{(2)}[B]
$$

with

$$
W^{(2)}[A]=\frac{1}{192 \pi^{2}} \int_{-\infty}^{\infty} \mathrm{d} \beta_{1} \int_{-\infty}^{\infty} \mathrm{d} \beta_{2} \frac{1}{\operatorname{ch} \beta_{0}} J\left(\beta_{1}, \beta_{2}, \beta_{0}\right) w_{1}\left(\beta_{1}, \beta_{2}, \beta_{0}\right),
$$

where $\beta_{0}$ is determined through $\operatorname{sh} \beta_{0}=-\operatorname{sh} \beta_{1}-\operatorname{sh} \beta_{2}$, and

$$
\begin{aligned}
W^{(2)}[B]\left(k_{1}, k_{2}, k_{3}, k_{4}\right)=\frac{1}{768 \pi^{2}} \frac{1}{T\left(\kappa_{1}+\kappa_{4}\right)} \int_{-\infty}^{\infty} \mathrm{d} \beta_{1} \int_{-\infty}^{\infty} \mathrm{d} \beta_{2} \int_{-\infty}^{\infty} \mathrm{d} \beta_{3} Z\left(\beta_{1}, \beta_{2}, \beta_{3}\right) \\
\left.\quad \times\left\{\delta\left(\beta_{1}, \beta_{2}, \beta_{3}, \kappa_{2}\right) \prod_{k=1}^{3} T\left(\beta_{k}+\kappa_{1}\right) \frac{\mathcal{P}}{T\left(\beta_{k}-\kappa_{4}\right)}+\left(\kappa_{2} \leftrightarrow \kappa_{3}, \kappa_{1} \leftrightarrow \kappa_{4}\right)\right\}, \quad \text { B. } 52\right)
\end{aligned}
$$

with

$$
Z\left(\beta_{1}, \beta_{2}, \beta_{3}\right)=J\left(\beta_{1}, \beta_{2}, \beta_{3}\right) w_{0}\left(\beta_{1}, \beta_{2}, \beta_{3}\right)
$$


We then see that $W^{(2)}[B]$ is a sum of two parts

$$
W^{(2)}[B]=W^{(2)}[B 1]+W^{(2)}[B 2]
$$

with

$$
\begin{aligned}
& W^{(2)}[B 1]=\frac{1}{64 \pi^{2}} \int_{-\infty}^{\infty} \mathrm{d} \beta_{1} \int_{-\infty}^{\infty} \mathrm{d} \beta_{2} \frac{1}{\operatorname{ch} \beta_{0}} Z\left(\beta_{1}, \beta_{2}, \beta_{0}\right) \frac{\mathcal{P}}{\operatorname{sh} \beta_{1}}, \\
& W^{(2)}[B 2]=\frac{1}{384 \pi^{2}} \int_{-\infty}^{\infty} \mathrm{d} \beta_{1} \int_{-\infty}^{\infty} \mathrm{d} \beta_{2} \frac{1}{\operatorname{ch} \beta_{0}} \frac{\partial}{\partial \beta_{3}}\left(\frac{Z\left(\beta_{1}, \beta_{2}, \beta_{3}\right)}{\operatorname{ch} \beta_{3}}\right)_{\beta_{3}=\beta_{0}} .
\end{aligned}
$$

Numerically this gives

$$
\begin{aligned}
W^{(2)}[A] & =4.41085(1) \times 10^{-4} \\
W^{(2)}[B 1] & =4.9600(1) \times 10^{-5} \\
W^{(2)}[B 2] & =1.1503(1) \times 10^{-5}
\end{aligned}
$$

and hence

$$
W^{(2)}=0.00050219(1)
$$

Finally we turn to the computation of $W^{(3)}$. Due to Eq. (B.49) it follows that

$$
v_{141}^{(I I, 2)}(0,0,0,0)=-2 v_{141}^{(I, 3)}(0,0,0,0)
$$

Now explicit computation yields

$$
\begin{aligned}
k^{(I)}(0,0, \beta,-\beta, 0,0) & =\pi^{-6}\left|\tau_{3}(0, \beta,-\beta)\right|^{2}\left(40 \beta^{2}+32 \pi^{2}\right) \\
k^{(I I I)}(\beta) & =-12 \pi^{-6}\left|\tau_{3}(0, \beta,-\beta)\right|^{2}\left(\beta^{2}+\pi^{2}\right) .
\end{aligned}
$$

So for $W^{(3)}$ we arrive at

$$
\begin{aligned}
W^{(3)} & =-\frac{\pi^{5}}{64} \int_{0}^{\infty} \frac{\operatorname{sh}^{6} \frac{\beta}{2} T^{6}(\beta)}{\operatorname{ch}^{5} \beta} \frac{\left(\beta^{2}+\pi^{2}\right)\left(4 \beta^{2}+\pi^{2}\right)\left(\beta^{2}+2 \pi^{2}\right)}{\beta^{6}\left(\beta^{2}+4 \pi^{2}\right)^{2}} \\
& =-0.0004682756 .
\end{aligned}
$$




\section{Computation of the $1-2-3$ contribution}

We use the results 3.57 3.61 with $m=3$, and begin with contribution $(I V)$ :

$$
\begin{array}{r}
v_{123}^{(I V)}\left(\kappa_{1}, \kappa_{2}, \kappa_{3}, \kappa_{4}\right) \sim \frac{1}{1536 \pi^{2}} \int \mathrm{d}^{3} \alpha \frac{\delta\left(\alpha_{1}, \alpha_{2}, \alpha_{3},-\kappa_{4}\right)}{\sum_{i=1}^{3} \operatorname{ch} \alpha_{i}} \\
\times \mathcal{G}^{(I V)}\left(-\kappa_{2}+i \pi_{-},-\kappa_{1}+i \pi_{-}, \alpha_{1}, \alpha_{2}, \alpha_{3}\right) .
\end{array}
$$

Here

$$
\mathcal{G}^{(I V)}(A)=\sum_{a_{1} a_{2} a_{3}} \mathcal{F}_{11 a_{1} a_{2} a_{3}}^{1}(A) \mathcal{F}_{a_{1} a_{2} a_{3}}^{1}\left(A^{\prime}\right)^{*}=T_{5}(A) T_{3}\left(A^{\prime}\right)^{*} g^{(I V)}(A)
$$

where $A$ stands for $\theta_{1}, \theta_{2}, \theta_{3}, \theta_{4}, \theta_{5}$ and $A^{\prime}$ for $\theta_{3}, \theta_{4}, \theta_{5}$. Note that $g^{(I V)}(A)$ is totally symmetric in the subset $A^{\prime}$.

We decompose the $1 /(x \pm i \epsilon)$ factors to obtain terms involving products of principle parts and delta-functions; only terms having less than three delta-functions contribute in the Lim procedure i.e.

$$
v_{123}^{(I V)}=\sum_{s=1}^{3} v_{123}^{(I V, s)}
$$

The terms are

$$
\begin{aligned}
& v_{123}^{(I V, 1)}\left(\kappa_{1}, \kappa_{2}, \kappa_{3}, \kappa_{4}\right) \sim \frac{1}{1536 \pi^{2}} T\left(\kappa_{1}-\kappa_{2}\right) \int \mathrm{d}^{3} \alpha \frac{\delta\left(\alpha_{1}, \alpha_{2}, \alpha_{3},-\kappa_{4}\right)}{\sum_{i=1}^{3} \operatorname{ch} \alpha_{i}} \\
& \quad \times g^{(I V)}\left(i \pi, i \pi, \alpha_{1}, \alpha_{2}, \alpha_{3}\right) \prod_{i<j} T^{2}\left(\alpha_{i}-\alpha_{j}\right) \prod_{k} \frac{\mathcal{P}}{T\left(\alpha_{k}+\kappa_{1}\right)} \frac{\mathcal{P}}{T\left(\alpha_{k}+\kappa_{2}\right)}, \\
& v_{123}^{(I V, 2)}\left(\kappa_{1}, \kappa_{2}, \kappa_{3}, \kappa_{4}\right) \sim-\frac{i}{128 \pi} \int \mathrm{d}^{2} \alpha \frac{\delta\left(-\kappa_{2}, \alpha_{1}, \alpha_{2},-\kappa_{4}\right)}{1+\sum_{i=1}^{2} \operatorname{ch} \alpha_{i}} \\
& \quad \times g^{(I V)}\left(i \pi, i \pi, 0, \alpha_{1}, \alpha_{2}\right) T^{2}\left(\alpha_{1}-\alpha_{2}\right) \prod_{k} T\left(\alpha_{k}+\kappa_{2}\right) \frac{\mathcal{P}}{T\left(\alpha_{k}+\kappa_{1}\right)}
\end{aligned}
$$

while for the term involving the product of two delta-functions one finds

$$
v_{123}^{(I V, 3)}\left(\kappa_{1}, \kappa_{2}, \kappa_{3}, \kappa_{4}\right)=O\left(\kappa^{3}\right)
$$

The contribution $(I V, 1)$ is antisymmetric in $\kappa_{1} \leftrightarrow \kappa_{2}$ and so it doesn't contribute in the sum over permutations. In the contribution $(I V, 2)$ we can take the $\kappa \rightarrow 0$ limit and obtain

$$
v_{123}^{(I V)}(0,0,0,0) \sim-\frac{i}{128 \pi} \int_{-\infty}^{\infty} \mathrm{d} \alpha \frac{T^{2}(2 \alpha)}{\operatorname{ch} \alpha[1+2 \operatorname{ch} \alpha]} g^{(I V)}(i \pi, i \pi, 0, \alpha,-\alpha) .
$$


Now turn to the $(I I I)$ contribution:

$$
v_{123}^{(I I I)}\left(\kappa_{1}, \kappa_{2}, \kappa_{3}, \kappa_{4}\right) \sim \frac{1}{64 \pi} \int \mathrm{d}^{2} \beta \frac{\delta\left(\beta_{1}, \beta_{2}, \kappa_{1}, \kappa_{2}\right)}{\sum_{i} \operatorname{ch} \beta_{i}\left[1+\sum_{j} \operatorname{ch} \beta_{j}\right]} \mathcal{G}^{(I I I)}\left(-\kappa_{1}+i \pi_{-}, \beta_{1}, \beta_{2}, \kappa_{3}\right)
$$

where

$$
\begin{aligned}
\mathcal{G}^{(I I I)}\left(\theta_{1}, \theta_{2}, \theta_{3}, \theta_{4}\right) & =\sum_{b_{1} b_{2}} \mathcal{F}_{1 b_{1} b_{2}}^{1}\left(\theta_{1}, \theta_{2}, \theta_{3}\right) \mathcal{F}_{b_{1} b_{2} 1}^{1}\left(\theta_{2}, \theta_{3}, \theta_{4}\right)^{*} \\
& =T_{3}\left(\theta_{1}, \theta_{2}, \theta_{3}\right) T_{3}\left(\theta_{2}, \theta_{3}, \theta_{4}\right)^{*} g^{(I I I)}\left(\theta_{1}, \theta_{2}, \theta_{3}, \theta_{4}\right)
\end{aligned}
$$

Here one can set the $\kappa_{i}$ to zero to obtain

$$
v_{123}^{(I I I)}(0,0,0,0) \sim \frac{1}{128 \pi} \int_{-\infty}^{\infty} \mathrm{d} \beta \frac{T^{2}(2 \beta)}{\operatorname{ch}^{2} \beta[1+2 \operatorname{ch} \beta]} g^{(I I I)}(i \pi, \beta,-\beta, 0) .
$$

For the $(I I)$ contribution:

$$
\begin{aligned}
& v_{123}^{(I I)}\left(\kappa_{1}, \kappa_{2}, \kappa_{3}, \kappa_{4}\right) \sim \frac{1}{256 \pi^{2}} \int \mathrm{d}^{2} \beta \frac{\delta\left(\beta_{1}, \beta_{2}, \kappa_{1}, \kappa_{2}\right)}{\operatorname{ch} \beta_{1}+\operatorname{ch} \beta_{2}} \\
& \int \mathrm{d}^{2} \alpha \frac{\delta\left(\beta_{1}, \alpha_{1}, \alpha_{2},-\kappa_{4}\right)}{\operatorname{ch} \beta_{1}+\sum_{i=1}^{2} \operatorname{ch} \alpha_{i}} \mathcal{G}^{(I I)}\left(-\kappa_{1}+i \pi_{-}, \beta_{1}, \beta_{2}, \alpha_{1}, \alpha_{2}\right),
\end{aligned}
$$

where

$$
\begin{aligned}
\mathcal{G}^{(I I)}(A) & =\sum_{b} \sum_{a_{1}, a_{2}} \mathcal{F}_{1 b b}^{1}\left(A^{\prime}\right) \mathcal{F}_{b a_{1} a_{2}}^{1}(B) \mathcal{F}_{b a_{1} a_{2}}^{1}\left(B^{\prime}\right)^{*} \\
& =T_{3}\left(A^{\prime}\right) T_{3}(B) T_{3}\left(B^{\prime}\right)^{*} g^{(I I)}(A)
\end{aligned}
$$

where $A$ stands for $\theta_{1}, \theta_{2}, \theta_{3}, \theta_{4}, \theta_{5} ; A^{\prime}$ stands for $\theta_{1}, \theta_{2}, \theta_{3}, B$ stands for $\theta_{3}+i \pi_{-}, \theta_{4}, \theta_{5}$, and $B^{\prime}$ stands for $\theta_{2}, \theta_{4}, \theta_{5}$. Making the familiar decomposition of the singular distributions one obtains:

$$
v_{123}^{(I I)}=\sum_{s=1}^{3} v_{123}^{(I I, s)}
$$

with

$$
\begin{aligned}
& v_{123}^{(I I, 1)}\left(\kappa_{1}, \kappa_{2}, \kappa_{3}, \kappa_{4}\right) \sim \frac{1}{256 \pi^{2}} \int \mathrm{d}^{2} \beta \frac{\delta\left(\beta_{1}, \beta_{2}, \kappa_{1}, \kappa_{2}\right)}{\operatorname{ch} \beta_{1}+\operatorname{ch} \beta_{2}} \\
& \quad \int \mathrm{d}^{2} \alpha \frac{\delta\left(\beta_{1}, \alpha_{1}, \alpha_{2},-\kappa_{4}\right)}{\operatorname{ch} \beta_{1}+\sum_{i=1}^{2} \operatorname{ch} \alpha_{i}} g^{(I I)}\left(-\kappa_{1}+i \pi, \beta_{1}, \beta_{2}, \alpha_{1}, \alpha_{2}\right) T^{2}\left(\alpha_{1}-\alpha_{2}\right) \\
& T\left(\beta_{1}-\beta_{2}\right) \prod_{i} \frac{\mathcal{P}}{T\left(\kappa_{1}+\beta_{i}\right)} \prod_{j} T\left(\beta_{1}-\alpha_{j}\right) \frac{\mathcal{P}}{T\left(\beta_{2}-\alpha_{j}\right)},
\end{aligned}
$$




$$
\begin{aligned}
& v_{123}^{(I I, 2)}\left(\kappa_{1}, \kappa_{2}, \kappa_{3}, \kappa_{4}\right) \sim \frac{i}{64 \pi} \int \mathrm{d}^{2} \beta \frac{\delta\left(\beta_{1}, \beta_{2}, \kappa_{1}, \kappa_{2}\right)}{\left[\operatorname{ch} \beta_{1}+\operatorname{ch} \beta_{2}\right]\left[1+\operatorname{ch} \beta_{1}+\operatorname{ch} \beta_{2}\right]} \\
& g^{(I I)}\left(-\kappa_{1}+i \pi, \beta_{1}, \beta_{2}, \beta_{2},-\kappa_{3}\right) T^{2}\left(\beta_{1}-\beta_{2}\right) \prod_{j} T\left(\beta_{j}+\kappa_{3}\right) \frac{\mathcal{P}}{T\left(\beta_{j}+\kappa_{1}\right)}, \\
& v_{123}^{(I I, 3)}\left(\kappa_{1}, \kappa_{2}, \kappa_{3}, \kappa_{4}\right) \sim \frac{i}{256 \pi} \int \mathrm{d}^{2} \alpha T^{2}\left(\alpha_{1}-\alpha_{2}\right) \\
& \quad\left\{\frac{\delta\left(-\kappa_{1}, \alpha_{1}, \alpha_{2},-\kappa_{4}\right)}{1+\sum_{i=1}^{2} \operatorname{ch} \alpha_{i}} g^{(I I)}\left(-\kappa_{1}+i \pi,-\kappa_{1},-\kappa_{2}, \alpha_{1}, \alpha_{2}\right) \prod_{j} T\left(\alpha_{j}+\kappa_{1}\right) \frac{\mathcal{P}}{T\left(\alpha_{j}+\kappa_{2}\right)}\right. \\
& \left.\quad+\frac{\delta\left(-\kappa_{2}, \alpha_{1}, \alpha_{2},-\kappa_{4}\right)}{1+\sum_{i=1}^{2} \operatorname{ch} \alpha_{i}} g^{(I I)}\left(-\kappa_{1}+i \pi,-\kappa_{2},-\kappa_{1}, \alpha_{1}, \alpha_{2}\right) \frac{\mathcal{P}}{T\left(\alpha_{j}+\kappa_{1}\right)}\right\},
\end{aligned}
$$

In the contributions $s=2,3$ we can set the $\kappa_{i}$ to zero to obtain

$$
\begin{aligned}
v_{123}^{(I I, 2)}(0,0,0,0) & \sim \frac{i}{128 \pi} \int_{-\infty}^{-\infty} \mathrm{d} \beta \frac{T^{2}(2 \beta)}{\operatorname{ch}^{2} \beta[1+2 \operatorname{ch} \beta]} g^{(I I)}(i \pi, \beta,-\beta,-\beta, 0), \\
v_{123}^{(I I, 3)}(0,0,0,0) & \sim \frac{i}{128 \pi} \int_{-\infty}^{-\infty} \mathrm{d} \alpha \frac{T^{2}(2 \alpha)}{\operatorname{ch} \alpha[1+2 \operatorname{ch} \alpha]} g^{(I I)}(i \pi, 0,0, \alpha,-\alpha),
\end{aligned}
$$

Finally for the $(I)$ contribution

$$
\begin{aligned}
& v_{123}^{(I)}\left(\kappa_{1}, \kappa_{2}, \kappa_{3}, \kappa_{4}\right) \sim \frac{1}{6144 \pi^{3}} \int \mathrm{d}^{2} \beta \frac{\delta\left(\beta_{1}, \beta_{2}, \kappa_{1}, \kappa_{2}\right)}{\operatorname{ch} \beta_{1}+\operatorname{ch} \beta_{2}} \\
& \int \mathrm{d}^{3} \alpha \frac{\delta\left(\alpha_{1}, \alpha_{2}, \alpha_{3},-\kappa_{4}\right)}{\sum_{i=1}^{3} \operatorname{ch} \alpha_{i}} \mathcal{G}^{(I)}\left(-\kappa_{1}+i \pi_{-}, \beta_{1}, \beta_{2}, \alpha_{1}, \alpha_{2}, \alpha_{3}\right),
\end{aligned}
$$

with

$$
\begin{aligned}
\mathcal{G}^{(I)}(A) & =\sum_{b} \sum_{a_{1}, a_{2}, a_{3}} \mathcal{F}_{1 b b}^{1}\left(A^{\prime}\right) \mathcal{F}_{b b a_{1} a_{2} a_{3}}^{1}(B) \mathcal{F}_{a_{1} a_{2} a_{3}}^{1}\left(B^{\prime}\right)^{*} \\
& =T_{3}\left(A^{\prime}\right) T_{5}(B) T_{3}\left(B^{\prime}\right)^{*} g^{(I)}(A)
\end{aligned}
$$

Here $A$ stands for $\theta_{1}, \theta_{2}, \theta_{3}, \theta_{4}, \theta_{5}, \theta_{6} ; A^{\prime}$ stands for $\theta_{1}, \theta_{2}, \theta_{3}, B$ stands for $\theta_{3}+i \pi_{-}, \theta_{2}+$ $i \pi, \theta_{4}, \theta_{5}, \theta_{6}$, and $B^{\prime}$ stands for $\theta_{4}, \theta_{5}, \theta_{6}$.

Then we rearrange to terms where after doing the $\beta_{2}$ integral the singularities in the $\beta_{1}$ integral all have negative imaginary parts

$$
v_{123}^{(I)}=\sum_{s=1}^{4} v_{123}^{(I, s)} .
$$


The terms are:

$$
\begin{aligned}
& v_{123}^{(I, 1)}\left(\kappa_{1}, \kappa_{2}, \kappa_{3}, \kappa_{4}\right) \sim-\frac{1}{6144 \pi^{3}} \int \mathrm{d}^{2} \beta \frac{\delta\left(\beta_{1}, \beta_{2}, \kappa_{1}, \kappa_{2}\right)}{\operatorname{ch} \beta_{1}+\operatorname{ch} \beta_{2}} \\
& \int \mathrm{d}^{3} \alpha \frac{\delta\left(\alpha_{1}, \alpha_{2}, \alpha_{3},-\kappa_{4}\right)}{\sum_{i=1}^{3} \operatorname{ch} \alpha_{i}} g^{(I)}\left(-\kappa_{1}+i \pi, \beta_{1}, \beta_{2}, \alpha_{1}, \alpha_{2}, \alpha_{3}\right) \\
& \quad \times \frac{T^{2}\left(\beta_{1}-\beta_{2}\right)}{T\left(\kappa_{1}+\beta_{1}+i \epsilon\right) T\left(\kappa_{1}+\beta_{2}-i \epsilon\right)} \frac{\prod_{i<j} T^{2}\left(\alpha_{i}-\alpha_{j}\right)}{\prod_{k} T\left(\beta_{1}+i \epsilon-\alpha_{k}\right) T\left(\beta_{2}-i \epsilon-\alpha_{k}\right)}, \\
& v_{123}^{(I, 2)}\left(\kappa_{1}, \kappa_{2}, \kappa_{3}, \kappa_{4}\right) \sim \frac{i}{512 \pi^{2}} \int \mathrm{d}^{2} \beta \frac{\delta\left(\beta_{1}, \beta_{2}, \kappa_{1}, \kappa_{2}\right)}{\operatorname{ch} \beta_{1}+\operatorname{ch} \beta_{2}} \\
& \quad \int \mathrm{d}^{2} \alpha \frac{\delta\left(\beta_{1}, \alpha_{1}, \alpha_{2},-\kappa_{4}\right)}{\operatorname{ch} \beta_{1}+\sum_{i=1}^{2} \operatorname{ch} \alpha_{i}} g^{(I)}\left(-\kappa_{1}+i \pi, \beta_{1}, \beta_{2}, \beta_{1}, \alpha_{1}, \alpha_{2}\right) \\
& \quad \frac{T\left(\beta_{1}-\beta_{2}\right) T^{2}\left(\alpha_{1}-\alpha_{2}\right) \prod_{i} T\left(\beta_{1}-\alpha_{i}\right)}{T\left(\kappa_{1}+\beta_{1}+i \epsilon\right) T\left(\kappa_{1}+\beta_{2}-i \epsilon\right) \prod_{j} T\left(\beta_{2}-i \epsilon-\alpha_{j}\right)}, \\
& \quad \times \frac{T^{2}\left(\alpha_{1}-\alpha_{2}\right) \prod_{i} T\left(\kappa_{2}+\alpha_{i}\right)}{\prod_{j} T\left(\kappa_{1}+i \epsilon+\alpha_{j}\right)} . \\
& v_{123}^{(I, 3)}\left(\kappa_{1}, \kappa_{2}, \kappa_{3}, \kappa_{4}\right) \sim \frac{i}{3072 \pi^{2}} T\left(\kappa_{1}-\kappa_{2}\right) \\
& \quad \int \mathrm{d}^{3} \alpha \frac{\delta\left(\alpha_{1}, \alpha_{2}, \alpha_{3},-\kappa_{4}\right)}{\sum_{i=1}^{3} \operatorname{ch} \alpha_{i}} g^{(I)}\left(-\kappa_{1}+i \pi,-\kappa_{2},-\kappa_{1}, \alpha_{1}, \alpha_{2}, \alpha_{3}\right) \\
& v_{123}^{(I, 4)}\left(\kappa_{1}, \kappa_{2}, \kappa_{3}, \kappa_{4}\right) \sim-\frac{1}{256 \pi} T\left(\alpha_{i}-\alpha_{j}\right) \\
& \prod_{k} T\left(-\kappa_{1}-i \epsilon-\kappa_{k}\right) T\left(-\kappa_{2}+i \epsilon-\alpha_{1}\right)
\end{aligned}
$$

As for the last contribution, it vanishes in the limit $\kappa_{i} \rightarrow 0$

$$
v_{123}^{(I, 4)}(0,0,0,0)=0
$$

For the contribution $(I, 1)$ we now perform the $\beta_{2}$ integral and shift the $\beta_{1}$ integral to larger imaginary part, after which we can send all the $\kappa_{i}$ to zero to obtain

$$
\begin{aligned}
& v_{123}^{(I, 1)}(0,0,0,0) \sim \frac{1}{512 \pi^{3}} \int_{-\infty+i \phi}^{+\infty+i \phi} \mathrm{d} \beta \frac{\operatorname{ch}^{4} \frac{\beta}{2}}{\operatorname{ch}^{4} \beta} \\
& \int_{0}^{\infty} \mathrm{d} u_{1} \int_{0}^{\infty} \mathrm{d} u_{2} T^{2}\left(u_{1}\right) T^{2}\left(u_{2}\right) T^{2}\left(u_{1}+u_{2}\right) M^{(3)}(u)^{-2}
\end{aligned}
$$




$$
\times g^{(I)}\left(i \pi, \beta,-\beta, \alpha_{1}, \alpha_{2}, \alpha_{3}\right) \prod_{k}\left(\frac{\operatorname{ch} \alpha_{k}+\operatorname{ch} \beta}{\operatorname{ch} \alpha_{k}-\operatorname{ch} \beta}\right),
$$

where the $\alpha_{k}$ are determined in terms of the $u$ 's as in Eq. (2.30).

For the $(I, 2)$ term we obtain

$$
v_{123}^{(I, 2)} \sim v_{123}^{(I, 5)}+v_{123}^{(I, 6)}+O(\kappa)
$$

where

$$
\begin{aligned}
& v_{123}^{(I, 5)}\left(\kappa_{1}, \kappa_{2}, \kappa_{3}, \kappa_{4}\right) \sim \frac{i}{512 \pi^{2}} \int \mathrm{d}^{2} \beta \frac{\delta\left(\beta_{1}, \beta_{2}, \kappa_{1}, \kappa_{2}\right)}{\operatorname{ch} \beta_{1}+\operatorname{ch} \beta_{2}} \\
& \quad \int \mathrm{d}^{2} \alpha \frac{\delta\left(\beta_{1}, \alpha_{1}, \alpha_{2},-\kappa_{4}\right)}{\operatorname{ch} \beta_{1}+\sum_{i=1}^{2} \operatorname{ch} \alpha_{i}} g^{(I)}\left(-\kappa_{1}+i \pi, \beta_{1}, \beta_{2}, \beta_{1}, \alpha_{1}, \alpha_{2}\right) \\
& \quad \times T\left(\beta_{1}-\beta_{2}\right) T^{2}\left(\alpha_{1}-\alpha_{2}\right) \prod_{i} \frac{\mathcal{P}}{T\left(\kappa_{1}+\beta_{i}\right)} \prod_{j} T\left(\beta_{1}-\alpha_{j}\right) \frac{\mathcal{P}}{T\left(\beta_{2}-\alpha_{j}\right)} \\
& v_{123}^{(I, 6)}\left(\kappa_{1}, \kappa_{2}, \kappa_{3}, \kappa_{4}\right) \sim-\frac{1}{128 \pi} \int \mathrm{d}^{2} \beta \frac{\delta\left(\beta_{1}, \beta_{2}, \kappa_{1}, \kappa_{2}\right)}{\sum_{i} \operatorname{ch} \beta_{i}\left[1+\sum_{j} \operatorname{ch} \beta_{j}\right]} \\
& g^{(I)}\left(-\kappa_{1}+i \pi, \beta_{1}, \beta_{2}, \beta_{1},-\beta_{2},-\kappa_{3}\right) T^{2}\left(\beta_{1}-\beta_{2}\right) \prod_{i} T\left(\beta_{i}+\kappa_{3}\right) \frac{\mathcal{P}}{T\left(\beta_{i}+\kappa_{1}\right)} .
\end{aligned}
$$

In the latter we can do the $\beta_{2}$ integral and set the $\kappa_{i}$ to zero to obtain

$$
v_{123}^{(I, 6)}(0,0,0,0) \sim-\frac{1}{256 \pi} \int_{-\infty}^{\infty} \mathrm{d} \beta \frac{T^{2}(2 \beta)}{\operatorname{ch}^{2} \beta[1+2 \operatorname{ch} \beta]} g^{(I)}(i \pi, \beta,-\beta, \beta,-\beta, 0) .
$$

Finally

$$
v_{123}^{(I, 3)}(0,0,0,0)=-\frac{1}{256 \pi} \int_{-\infty}^{\infty} \mathrm{d} \alpha \frac{T^{2}(2 \alpha)}{\operatorname{ch} \alpha[1+2 \operatorname{ch} \alpha]} g^{(I)}(i \pi, 0,0, \alpha,-\alpha, 0) .
$$

Summarizing the results we have

$$
v_{123}(0,0,0,0)=\sum_{j=1}^{5} V^{(j)}
$$

where the five terms are as follows:

$$
V^{(1)}=v_{123}^{(I, 1)}(0,0,0,0)
$$


given in Eq. (C.28). Further

$$
\begin{aligned}
V^{(2)} & =v_{123}^{(I I)}(0,0,0,0)+v_{123}^{(I V)}(0,0,0,0) \\
& =\frac{1}{128 \pi} \int_{-\infty}^{\infty} \mathrm{d} \beta \frac{T^{2}(2 \beta)}{\operatorname{ch}^{2} \beta} g^{(2)}(\beta),
\end{aligned}
$$

where

$$
g^{(2)}(\beta)=\frac{g^{(I I I)}(i \pi, \beta,-\beta, 0)-i g^{(I V)}(i \pi, i \pi, 0, \beta,-\beta) \operatorname{ch} \beta}{1+2 \operatorname{ch} \beta} .
$$

Next

$$
\begin{aligned}
V^{(3)}= & v_{123}^{(I, 5)}(0,0,0,0)+v_{123}^{(I I, 1)}(0,0,0,0) \\
= & -\frac{1}{512 \pi^{2}} \int_{-\infty}^{\infty} \mathrm{d} \beta \frac{\operatorname{ch}^{2} \frac{\beta}{2}}{\operatorname{ch}^{3} \beta} \frac{\mathcal{P}}{T(\beta)} \int \mathrm{d}^{2} \alpha \frac{\delta\left(\beta, \alpha_{1}, \alpha_{2}\right)}{\operatorname{ch} \beta+\sum_{i=1}^{2} \operatorname{ch} \alpha_{i}} g^{(3)}\left(\beta, \alpha_{1}, \alpha_{2}\right) \\
& \times T^{2}\left(\alpha_{1}-\alpha_{2}\right) \prod_{j} T\left(\alpha_{j}-\beta\right) \frac{\mathcal{P}}{T\left(\alpha_{j}+\beta\right)},
\end{aligned}
$$

where

$$
g^{(3)}\left(\beta, \alpha_{1}, \alpha_{2}\right)=2 g^{(I I)}\left(i \pi, \beta,-\beta, \alpha_{1}, \alpha_{2}\right)+i g^{(I)}\left(i \pi, \beta,-\beta, \beta, \alpha_{1}, \alpha_{2}\right) .
$$

Further

$$
\begin{aligned}
V^{(4)} & =v_{123}^{(I, 3)}(0,0,0,0)+v_{123}^{(I I, 3)}(0,0,0,0) \\
& =-\frac{1}{256 \pi} \int_{-\infty}^{\infty} \mathrm{d} \alpha \frac{T^{2}(2 \alpha)}{\operatorname{ch} \alpha[1+2 \operatorname{ch} \alpha]} g^{(4)}(\alpha),
\end{aligned}
$$

where

$$
g^{(4)}(\alpha)=-i g^{(3)}(0, \alpha,-\alpha)
$$

Finally

$$
\begin{aligned}
V^{(5)} & =v_{123}^{(I, 6)}(0,0,0,0)+v_{123}^{(I I, 2)}(0,0,0,0), \\
& =-\frac{1}{256 \pi} \int_{-\infty}^{\infty} \mathrm{d} \beta \frac{T^{2}(2 \beta)}{\operatorname{ch}^{2} \beta[1+2 \operatorname{ch} \beta]} g^{(5)}(\beta),
\end{aligned}
$$

where

$$
g^{(5)}(\beta)=-i g^{(3)}(\beta,-\beta, 0) .
$$




\section{Case $n=1$ :}

Here we have simply

$$
\begin{aligned}
g^{(I)}(A) & =-16, & & g^{(I I)}(B)=8 i, \\
g^{(I I I)}(C) & =4, & & g^{(I V)}(D)=8 i,
\end{aligned}
$$

and so

$$
g^{(2)}(\beta)=4, \quad g^{(r)}=0, r=3,4,5 .
$$

Thus

$$
\begin{aligned}
& V^{(2)}=\frac{1}{32 \pi} \int_{-\infty}^{\infty} \mathrm{d} \beta \frac{\operatorname{sh}^{2} \beta}{\operatorname{ch}^{4} \beta}=\frac{1}{48 \pi}, \\
& V^{(1)}=-\frac{1}{128 \pi^{3}} \int_{0}^{\infty} \mathrm{d}^{2} u T^{2}\left(u_{1}\right) T^{2}\left(u_{2}\right) T^{2}\left(u_{1}+u_{2}\right) \frac{S\left(u_{1}, u_{2}\right)}{M^{(3)}(u)^{2}},
\end{aligned}
$$

with

$$
S\left(u_{1}, u_{2}\right)=\int_{-\infty+i \phi}^{+\infty+i \phi} \mathrm{d} \beta \frac{[1+\operatorname{ch} \beta]^{2}}{\operatorname{ch}^{4} \beta} \prod_{k=1}^{3}\left(\frac{\operatorname{ch} \alpha_{k}+\operatorname{ch} \beta}{\operatorname{ch} \alpha_{k}-\operatorname{ch} \beta}\right) .
$$

Numerically we find

$$
V^{(1)}=-0.000842721(1) \text {. }
$$

\section{Case $n=3$ :}

First we have

$$
V^{(1)}=\frac{\pi^{9}}{32768} \int_{0}^{\infty} \mathrm{d}^{2} u\left|\psi\left(u_{1}\right) \psi\left(u_{2}\right) \psi\left(u_{1}+u_{2}\right)\right|^{2} M^{(3)}(u)^{-2} S\left(u_{1}, u_{2}\right),
$$

where

$$
|\psi(u)|^{2}=\frac{u^{2}+\pi^{2}}{u^{2}\left(u^{2}+4 \pi^{2}\right)} T^{4}(u)
$$

and

$$
\begin{aligned}
& S\left(u_{1}, u_{2}\right)=\int_{-\infty+i \phi}^{+\infty+i \phi} \mathrm{d} \beta \frac{(1+\operatorname{ch} \beta)^{4}}{\operatorname{ch}^{6} \beta} h^{(I)}\left(i \pi, \beta,-\beta, \alpha_{1}, \alpha_{2}, \alpha_{3}\right) \\
& \quad \frac{\left(4 \beta^{2}+\pi^{2}\right)}{\left(\beta^{2}+\pi^{2}\right)^{3}} \prod_{k} \frac{\alpha_{k}^{2}-\beta^{2}}{\left(\alpha_{k}^{2}-\beta^{2}\right)^{2}+2 \pi^{2}\left(\alpha_{k}^{2}+\beta^{2}\right)+\pi^{4}}\left(\frac{\operatorname{ch} \alpha_{k}+\operatorname{ch} \beta}{\operatorname{ch} \alpha_{k}-\operatorname{ch} \beta}\right)^{2} .
\end{aligned}
$$

Numerically this gives

$$
V^{(1)}=-0.000844527(1)
$$


Doing the contractions yields

$$
\begin{aligned}
g^{(I I I)}(i \pi, \beta,-\beta, 0) & =\frac{\pi^{6}}{4} \frac{\left(2 \beta^{2}+\pi^{2}\right)}{\left(\beta^{2}+\pi^{2}\right)^{2}} \frac{T^{2}(2 \beta)}{\beta^{2}}, \\
g^{(I V)}(i \pi, i \pi, 0, \beta,-\beta) & =2 i g^{(I I I)}(i \pi, \beta,-\beta, 0) .
\end{aligned}
$$

Thus

$$
g^{(2)}(\beta)=g^{(I I I)}(i \pi, \beta,-\beta, 0),
$$

and

$$
V^{(2)}=\frac{\pi^{5}}{512} \int_{-\infty}^{\infty} \mathrm{d} \beta \frac{\operatorname{sh}^{4} \beta}{\beta^{2} \operatorname{ch}^{6} \beta} \frac{\left(2 \beta^{2}+\pi^{2}\right)\left(4 \beta^{2}+\pi^{2}\right)}{\left(\beta^{2}+\pi^{2}\right)^{2}\left(\beta^{2}+4 \pi^{2}\right)}=0.0074380765 .
$$

Next by explicit computation one verifies

$$
g^{(4)}(\alpha)=0, \quad g^{(5)}(\beta)=-g^{(5)}(-\beta),
$$

and thus'

$$
V^{(4)}=V^{(5)}=0 .
$$

It remains to compute $V^{(3)}$. Shifting the $\alpha_{1}$ integral we obtain the representation

$$
\begin{aligned}
& V^{(3)}=\frac{1}{256 \pi^{2}} \int_{-\infty}^{\infty} \mathrm{d} \beta \int_{-\infty}^{\infty} \mathrm{d} \alpha \frac{\mathcal{P}}{\operatorname{sh}^{\frac{\beta}{2}}} \frac{\operatorname{ch}^{2} \frac{\beta}{2}}{\operatorname{ch}^{3} \beta} \frac{\mathcal{P}}{T(\alpha)} \\
& \quad \frac{g^{(3)}\left(\beta, \alpha_{1}, \alpha_{2}\right) \operatorname{ch} \frac{\alpha_{1}}{2} \operatorname{ch} \frac{\alpha_{2}+\beta}{2}}{\operatorname{ch} \alpha_{2} \operatorname{ch} \frac{\alpha_{1}-\alpha_{2}}{2}\left[\operatorname{ch} \beta+\operatorname{ch} \alpha_{1}+\operatorname{ch} \alpha_{2}\right]} T\left(\alpha_{1}-\alpha_{2}\right) T\left(\alpha_{1}-\beta\right) T\left(\alpha_{2}-\beta\right) \operatorname{ch} \frac{\alpha_{1}}{2},(\mathrm{C} .58)
\end{aligned}
$$

where

$$
\alpha_{1}=\alpha-\beta,
$$

and $\alpha_{2}$ is determined by

$$
\operatorname{sh} \alpha_{2}=-\operatorname{sh} \beta-\operatorname{sh} \alpha_{1} .
$$

Numerically this gives

$$
V^{(3)}=-0.000125112(1) \text {. }
$$

${ }^{*}$ Eq. (C.57) is perhaps true for all $n$ but we have not verified this conjecture 


\section{Building blocks of form factors}

In form factor calculations one often encounters the problem of finding an analytic function $f(\theta)$ satisfying

$$
\begin{aligned}
f(\theta) & =\sigma(\theta) f(-\theta), \\
f(i \pi-\theta) & =f(i \pi+\theta),
\end{aligned}
$$

for given $\sigma(\theta)$. If $\sigma(\theta)$ has the Fourier representation

$$
\sigma(\theta)=e^{i \delta(\theta)}, \quad \delta(\theta)=2 \int_{0}^{\infty} \frac{\mathrm{d} \omega}{\omega} \sin (\theta \omega) \tilde{k}(\omega)
$$

with some kernel function $\tilde{k}(\omega)$ then the 'minimal' solution of (D.1) is given by [4]

$$
f(\theta)=e^{\Delta(\theta)}, \quad \Delta(\theta)=\int_{0}^{\infty} \frac{\mathrm{d} \omega}{\omega} \frac{\operatorname{ch} \omega(\pi+i \theta)-1}{\operatorname{sh} \pi \omega} \tilde{k}(\omega) .
$$

The function $\Delta(\theta)$ has the following properties. If $\tilde{k}(\omega) \sim e^{-z \omega}(z>0)$ for $\omega \rightarrow \infty$ then $\Delta(\theta)$ is analytic for $-z<\operatorname{Im} \theta<2 \pi+z$ and for real $\theta \rightarrow \infty$

$$
\operatorname{Re} \Delta(\theta) \sim \Delta(i \pi+\theta) \sim-\frac{\theta}{2} \tilde{k}(0)-\frac{\ln \theta}{\pi} \tilde{k}^{\prime}(0)+\text { const. }
$$

We encountered in Section 6 the following special case: for some (positive, real) parameter $\alpha$

$$
\sigma_{\alpha}(\theta)=e^{i \delta_{\alpha}(\theta)}=\frac{(1+\alpha) i \pi+\theta}{(1+\alpha) i \pi-\theta},
$$

corresponding to the kernel

$$
\tilde{k}_{\alpha}(\omega)=-e^{-\pi \omega(1+\alpha)} .
$$

We denote the corresponding solution by $\Delta_{\alpha}(\theta)$. 


\section{References}

[1] C. Newman, Comm. Math. Phys 41 (1975) 1.

[2] J. Glimm and A. Jaffe, Quantum Physics, 2nd ed., Springer 1987.

[3] A.B. and Al.B. Zamolodchikov, Ann. Phys. 120 (1979) 253; Nucl. Phys. B133 (1978) 525 .

[4] M. Karowski and P. Weisz, Nucl. Phys. B139 (1978) 455.

[5] M. Karowski, in: Field theoretical methods in particle physics, 1980, ed. W. Rühl, p. 307.

[6] F.A. Smirnov: Form factors in Completely Integrable Models of Quantum Field Theory, World Scientific, 1992.

[7] M. Falcioni, G. Martinelli, M. L. Paciello, G. Parisi and B. Taglienti, Nucl. Phys. B225 (1983) 313.

[8] J. Kim, Phys. Lett. B345 (1995) 469.

[9] J. Balog, M. Niedermaier, F. Niedermayer, A. Patrascioiu, E. Seiler and P. Weisz, Phys. Rev. D60 (1999) 094508.

[10] M. Campostrini, A. Pelissetto, P. Rossi and E. Vicari, Nucl. Phys. B459 (1996) 207; Nucl. Phys. Proc. Suppl. 47 (1996) 751.

[11] M. Campostrini, A. Pelissetto, P. Rossi and E. Vicari, Phys. Phys. D54 (1996) 1782; Nucl. Phys. Proc. Suppl. 47 (1996) 755.

[12] P. Butera and M. Comi, Phys. Rev. B54 (1996) 15828.

[13] A. Pelissetto and E. Vicari, Nucl. Phys. B519 (1998) 626.

[14] J. Balog, M. Niedermaier, F. Niedermayer, A. Patrascioiu, E. Seiler and P. Weisz, in preparation.

[15] M. Niedermaier, Parametric holomorphy? Triviality versus duality in Sinh-Gordon, hep-th/9909170.

[16] M. Niedermaier, Nucl. Phys. B519 (1998) 517. 
[17] M. Lüscher, NPB135 (1978) 1.

[18] P. Hasenfratz and F. Niedermayer, Nucl. Phys. B414 (1994) 785.

[19] T.T. Wu, B. McCoy, C. Tracy and E. Baruch, Phys. Rev. B13 (1976) 316.

[20] M. Sato, T. Miwa and M. Jimbo, Proc. Jpn. Acad. 53A (1997) 6.

[21] B. McCoy, hep-th/9403084.

[22] B. Berg, M. Karowski and P. Weisz, Phys. Rev. D19 (1979) 2477.

[23] M. Campostrini, A. Pelissetto, P. Rossi and E. Vicari, Phys. Rev. B54 (1996) 7301.

[24] O. Babelon and D. Bernard, Phys. Lett. B288 (1992) 113.

[25] G.A. Baker, Phys. Rev. B15 (1977) 1552.

[26] J.C. LeGuillon and J. Zinn-Justin, Phys. Rev. Lett 39 (1977) 95; Phys. Rev. B21 (1980) 3976.

[27] J. Kim and A. Patrascioiu, Phys. Rev D47 (1993) 2588.

[28] A.B. Zamolodchikov, Int. J. of Mod. Phys. A3 (1988) 743;

G. Delfino, Phys. Lett. B419 (1998) 291.

[29] J.M. Kosterlitz and D.J. Thouless, J. Phys. C6 (1973) 1181;

J.M. Kosterlitz, J. Phys. C7 (1974) 1046.

[30] J. Zinn-Justin, Quantum Field Theory and Critical Phenomena, Oxford, 1989.

[31] H. Babujian, A. Fring, M. Karowski and A. Zapletal, Nucl. Phys. B538 (1999) 535.

[32] C.H. Woo, Phys. Rev. D20 (1979) 1880.

[33] A.P. Prudnikov, Yu.A. Brichkov and O.I. Marichev, Integrali i ryadi (Nauka, Moskow, 1981) formulae 2.4.22.27.

[34] J. Balog and M. Niedermaier, Nucl. Phys. B500 (1997) 421; Phys. Rev. Lett. 78 (1997) 4151.

[35] J. Balog, M. Niedermaier and T. Hauer, Phys. Lett. B386 (1996) 224.

[36] M. Campostrini, A. Pelissetto, P. Rossi and E. Vicari, Phys. Lett. B402 (1997) 141. 
[37] P. Rossi and E. Vicari, private communication.

[38] P. Butera, private communication.

[39] M. Lüscher and U. Wolff, Nucl. Phys. B339 (1990) 222.

[40] P. Hasenfratz, M. Maggiore and F. Niedermayer, Phys. Lett. B245 (1990) 522.

[41] M. Hasenbusch, hep-lat/9408019. 